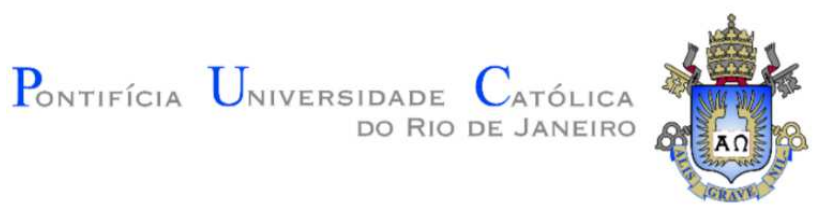

Ana Carolina Guimarães Costa

\title{
Controle Preditivo Baseado em Modelo Não- Linear Aplicado a uma Coluna Despropanizadora
}

\section{Dissertação de Mestrado}

Dissertação apresentada como requisito parcial para obtenção do grau de Mestre pelo Programa de PósGraduação em Engenharia de Materiais e de Processos Químicos e Metalúrgicos do Departamento de Engenharia Química e de Materiais da PUC-Rio.

Orientador: Prof. Brunno Ferreira dos Santos Co-orientadora: Dra. Simone de Carvalho Miyoshi

Rio de Janeiro Setembro de 2019 


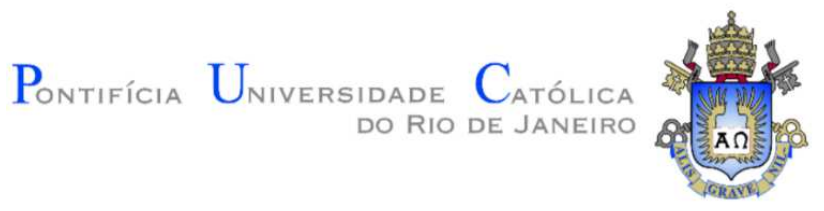

Ana Carolina Guimarães Costa

\section{Controle Preditivo Baseado em Modelo Não- Linear Aplicado a uma Coluna Despropanizadora}

Dissertação apresentada como requisito parcial para obtenção do grau de Mestre pelo Programa de Pósgraduação em Engenharia de Materiais e de Processos Químicos e Metalúrgicos da PUC-Rio. Aprovada pela Comissão Examinadora abaixo.

Prof. Brunno Ferreira dos Santos

Orientador Departamento de Engenharia Química e Materiais - PUC-Rio

Dra. Simone de Carvalho Miyoshi Co-orientadora Departamento de Engenharia Química - UFSCar

Prof. Filipe Alves Coelho Departamento de Engenharia Química - USF

Prof. Helon Vicente Hultmann Ayala Departamento de Engenharia Mecânica - PUC-Rio 
Todos os direitos reservados. É proibida a reprodução total ou parcial do trabalho sem autorização da universidade, do autor e do orientador.

\section{Ana Carolina Guimarães Costa}

Graduou-se em Engenharia Química pela Pontifícia Universidade Católica do Rio de Janeiro (PUC-Rio) em 2017. É Analista de Soluções Avançadas na Yokogawa South América.

Ficha Catalográfica

Costa, Ana Carolina Guimarães

Controle Preditivo Baseado em Modelo Não-linear Aplicado a uma Coluna Despropanizadora / Ana Carolina Guimarães Costa ; orientador: Brunno Ferreira dos Santos ; co-orientadora: Simone de Carvalho Miyoshi. -2019.

130 f. : il. color. ; $30 \mathrm{~cm}$

Dissertação (mestrado)-Pontifícia Universidade Católica do Rio de Janeiro, Departamento de Engenharia Química e de Materiais, 2019.

Inclui bibliografia

1. Engenharia Química e de Materiais - Teses. 2. Coluna de destilação. 3. Despropanizadora. 4. Controle Preditivo baseado em Modelo Não-Linear (NMPC). 5. Modelo de Hammerstein. 6. Redes Neurais Artificiais (RNA). I. Santos, Brunno Ferreira dos. II. Miyoshi, Simone de Carvalho. III. Pontifícia Universidade Católica do Rio de Janeiro. Departamento de Engenharia Química e de Materiais. IV. Título.

CDD: 620.11 
Para meu pai Ramon, por todo o amor incondicional, pelo apoio e confiança. 


\section{Agradecimentos}

Aos professores Brunno Santos e Simone Miyoshi, pelos pacientes anos de orientação, minha gratidão e admiração. Foram eles que me deram recursos e ferramentas para evoluir um pouco mais todos os dias.

À Debora Zide, consultora de Sistemas Avançados na White Martins, por ter me sugerido o tema e incentivado para realizar este trabalho.

Aos colegas do LADES (UFRJ) pelo apoio.

À toda a minha família, que me incentivou nos estudos e se orgulha de cada conquista minha. Um especial agradecimento à minha tia Miriam, minha prima Liv e minha prima Thânia. Também sou grata à minha mãe Raquel que, com certeza, está vibrando lá no céu.

Às minhas amigas queridas que me acalmam nos momentos difíceis, Clara, Maria Cláudia, Joana e Erika. O que seria de mim sem vocês.

O presente trabalho foi realizado com o apoio da Coordenação de Aperfeiçoamento de Pessoal de Nível Superior - Brasil (CAPES) - Código de Financiamento 001. 


\section{Resumo}

Costa, Ana Carolina Guimarães; Santos, Brunno; Miyoshi, Simone de Carvalho. Controle Preditivo Baseado em Modelo Não-linear Aplicado a uma Coluna Despropanizadora. Rio de Janeiro, 2019. 130p. Dissertação de Mestrado - Departamento de Engenharia Química e de Materiais, Pontifícia Universidade Católica do Rio de Janeiro.

Este trabalho tem como objetivo estudar estratégias de Controle Preditivo baseado em Modelo Não-Linear (NMPC) aplicadas a uma coluna de destilação despropanizadora simulada. Essas colunas são empregadas em unidades de processamento de gás natural (UPGNs) para a separação do produto propano do butano. Colunas de destilação possuem características particularmente desafiadoras sob o ponto de vista de controle, como: não-linearidades, grandes constantes de tempo, atraso, restrições de variáveis e inversão do sinal de ganho estático. Como as medidas de composição frequentemente possuem atrasos e dados esparsos, os sistemas de controle convencionais não são capazes de controlar a composição diretamente e possuem dificuldade em manter os produtos dentro das especificações. Contudo, controladores baseados em modelo possuem a habilidade de prever a composição através do modelo interno do processo, além de serem capazes de lidar com restrições. Na literatura, nenhuma aplicação do modelo de Hammerstein modificado para coluna de destilação ou para sistemas multivariáveis foi encontrada, sendo esta uma novidade. Desta forma, foram estudadas três estratégias de controle: controle PID tradicional, NMPC com modelo de Hammerstein modificado (H-NMPC) e NMPC com modelo por Redes Neurais (NN-NMPC). O sistema estudado foi identificado de forma a se obter valores numéricos adequados aos parâmetros dos modelos. A identificação dos parâmetros dos modelos e os algoritmos de NMPC foram implementados no ambiente MATLAB. A coluna de destilação foi simulada usando o Aspen Plus Dynamics ${ }^{\circledR}$. Como resultado, o H-NMPC teve o melhor desempenho de controle ao rastrear diferentes trajetórias de referência, a desacoplar as variáveis controladas e a rejeitar os distúrbios. Além disso, esta

apresentou maior rapidez computacional comparado com a estratégia NNNMPC.

\section{Palavras-chave}

Coluna de destilação; Despropanizadora; Controle Preditivo baseado em Modelo Não-Linear (NMPC); Modelo de Hammerstein; Redes Neurais Artificiais (RNA) 


\section{Abstract}

Costa, Ana Carolina Guimarães; Santos, Brunno Ferreira dos (Advisor); Miyoshi, Simone de Carvalho (Co-advisor). Nonlinear Model Predictive Control Applied to a Depropanizer Column. Rio de Janeiro, 2019. 130p. Dissertação de Mestrado - Departamento de Engenharia Química e de Materiais, Pontifícia Universidade Católica do Rio de Janeiro.

This work aims to study strategies of Nonlinear Model Predictive Control (NMPC) applied to a simulated depropanizer distillation column. These columns are used in natural gas processing units (NGPUs) for the separation of the product propane from butane. Distillation columns have particularly challenging features from the control point of view, such as: nonlinearities, large time constants, delay, variable constraints and static gain signal inversion. Because compositional measures often have delays and sparse data, conventional control systems are not able to control composition directly and have difficulty keeping products within specifications. However, model-based controllers predict composition through the internal process model, besides being able to handle constraints. In the literature, no applications of the modified Hammerstein model for distillation column or multivariable systems was found, so this is a novelty. Therefore, three control strategies were studied: traditional PID control, NMPC with modified Hammerstein model (H-NMPC) and NMPC with neural network model (NN-NMPC). The studied system was identified in order to obtain adequate numerical values of the model parameters. The model identification and the NMPC algorithms were implemented in the MATLAB environment. The distillation column was simulated using Aspen Plus Dynamics ${ }^{\circledR}$. As a result, the H-NMPC provided better control performance for different setpoint tracking, control variables decoupling, and disturbance rejection. Furthermore, it presented faster computational speed compared to NN-NMPC.

\section{Keywords}

Distillation Column; Depropanizer; Nonlinear Model Predictive Control (NMPC); Hammerstein Model; Artificial Neural Network (ANN) 


\section{Sumário}

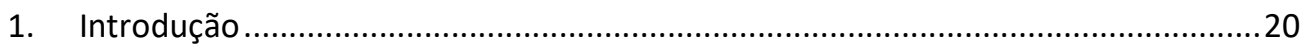

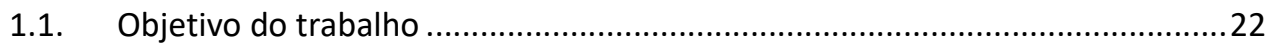

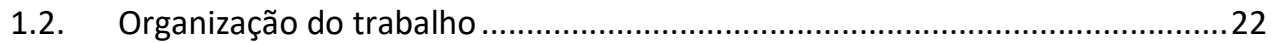

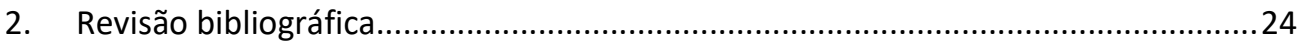

2.1. Unidade de Processamento de Gás Natural (UPGN) ......................................24

2.1.1. Especificação dos produtos ........................................................................26

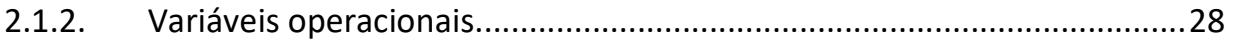

2.1.3. Estruturas de controle para coluna de destilação........................................29

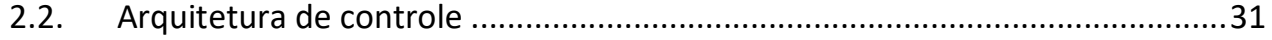

2.3. Controle Preditivo Baseado em Modelo (MPC) …..............................................33

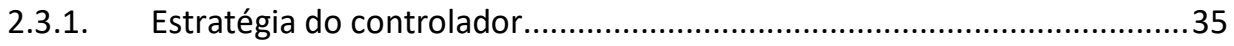

2.3.2. Formulação do modelo linear em espaço de estado .................................37

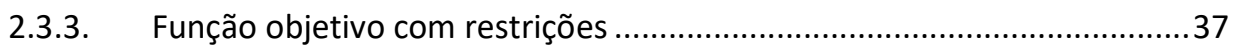

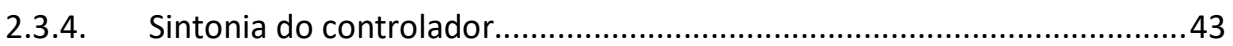

2.4. Controle Preditivo Baseado em Modelo Não-Linear (NMPC) ...........................4

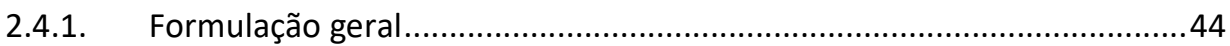

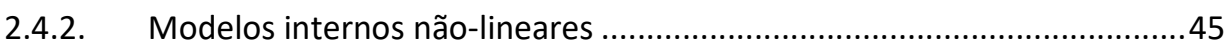

2.4.3. Controle Preditivo baseado em Modelo de Hammerstein Modificado ....49

2.4.4. Controle Preditivo baseado em Modelo de Redes Neurais .......................58

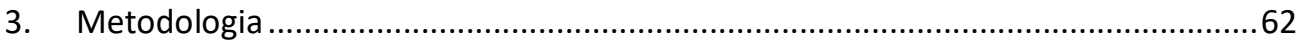

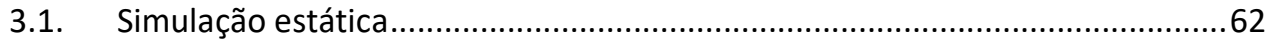

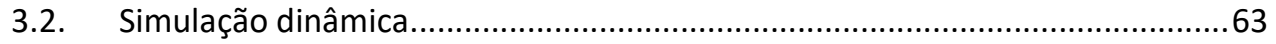

3.2.1. Estruturas de controle ..............................................................................64

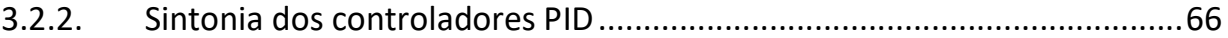

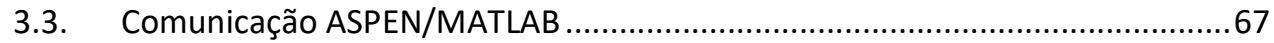

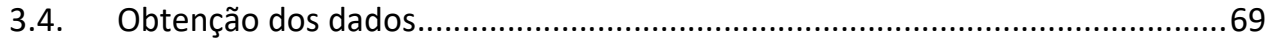

3.5. Modelagem e identificação dos parâmetros dos modelos ..................................69

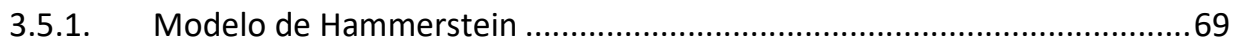

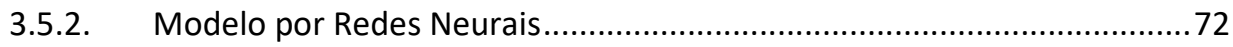

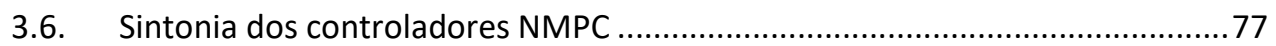

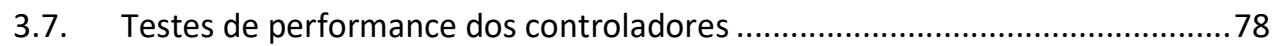

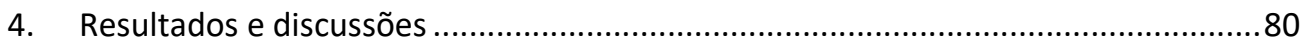

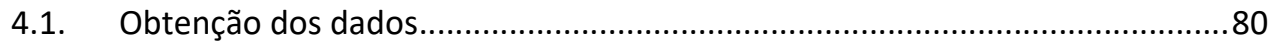


4.2. Modelagem e identificação dos parâmetros dos modelos.............................81

4.2.1. Modelo de Hammerstein ................................................................ 81

4.2.2. Modelo por Redes Neurais ....................................................... 88

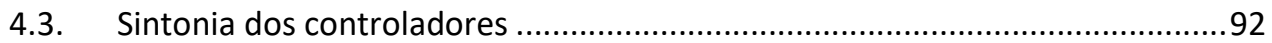

4.3.1. Sintonia do controlador PID ...................................................... 92

4.3.2. Sintonia dos controladores NMPC …................................................ 93

4.4. Testes de performance dos controladores ................................................. 106

4.4.1. Teste de rastreamento da trajetória de referência e desacoplamento das variáveis controladas............................................................................... 106

4.4.2. Teste de rejeição de Distúrbios .....................................................111

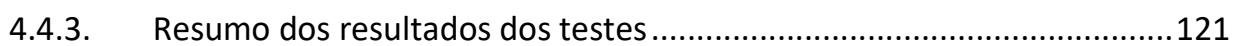

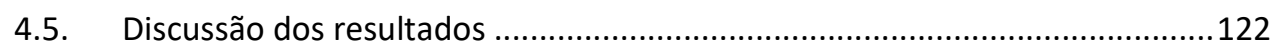

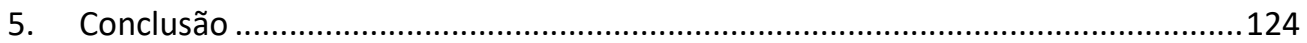

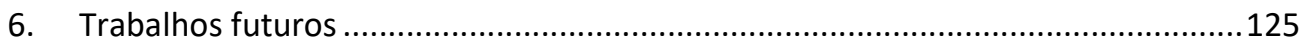

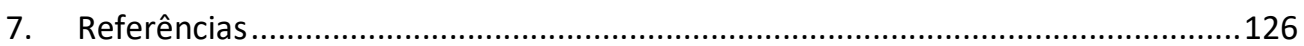




\section{Lista de Figuras}

Figura 1: Esquema simplificado das colunas de destilação de uma UPGN. ………........... 25

Figura 2: Graus de liberdade de uma coluna de destilação. ............................................ 30

Figura 3: Coluna de destilação controlada com a estrutura LV, com circuito de temperatura em V e um controle feedforward em L/F. Fonte: Skogestad, 2007.

Figura 4: Hierarquia de controle típica em uma planta de processos quimicos.

Adaptado de DARBY et al., (2011). .......................................................................... 32

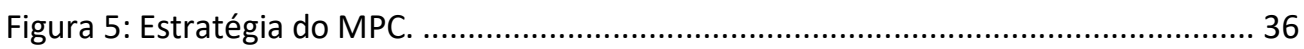

Figura 6: Modelo de Hammerstein modificado (MIYOSHI, 2016).................................... 51

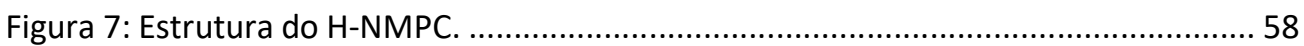

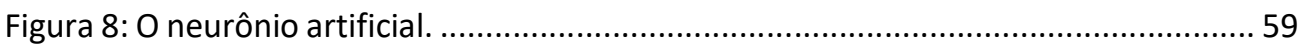

Figura 9: Estrutura do modelo de redes neurais recursivo para implementação do NN-

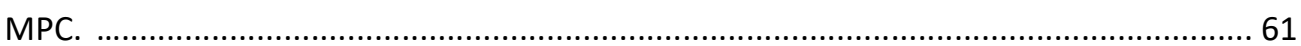

Figura 10: Simulação da coluna de destilação em Aspen Plus. ........................................ 63

Figura 11: Simulação da coluna de destilação em Aspen Plus Dynamics para simulação

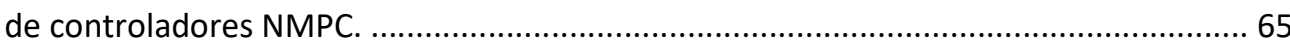

Figura 12: Simulação da coluna de destilação em Aspen Plus Dynamics para simulação

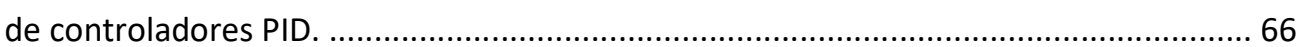

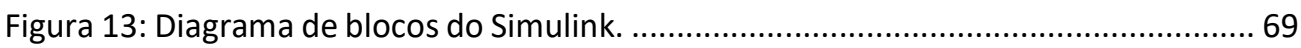

Figura 14: Estrutura do modelo de redes neurais recursivo proposta para

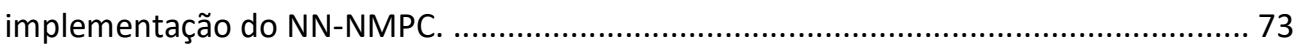

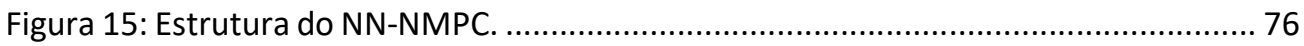

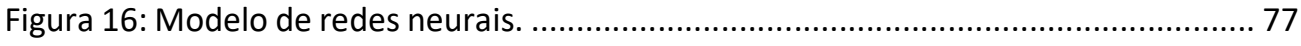

Figura 17: Gráficos dos setpoints das variáveis manipuladas $\mathrm{R}$ e $\mathrm{T}$ e as respostas das

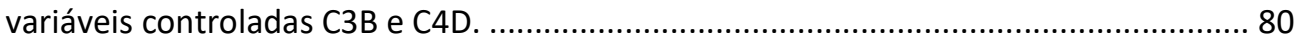

Figura 18: Superfície para a saida C3B em função de T e R. ........................................... 81

Figura 19:Superfície para a saida C4D em função de T e R. ............................................. 82

Figura 20: Convergência do SSE para um valor mínimo a cada interação da estimação

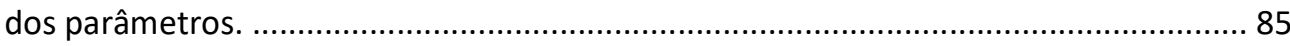

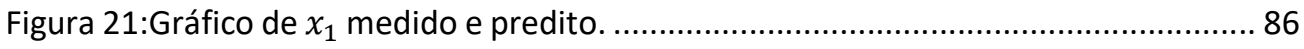

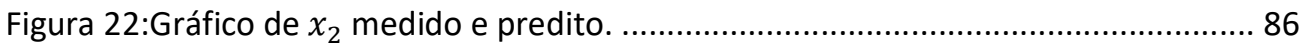

Figura 23: Gráfico do desempenho do estimador na presença de ruído de medição. ..... 88

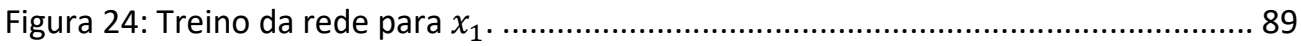




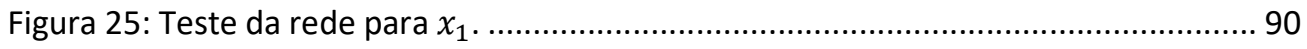

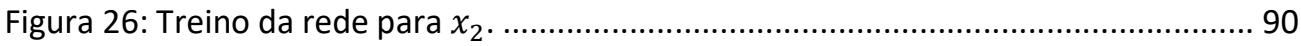

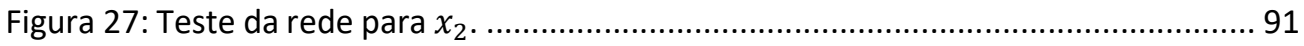

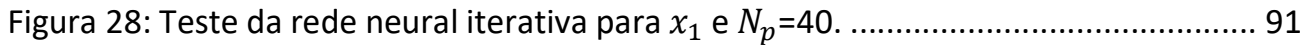

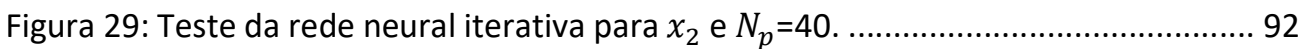

Figura 30: Tempo de resposta para as duas variáveis controladas. ............................ 93

Figura 31: Sintonia do $N_{C}$ para saída C3B. ....................................................... 94

Figura 32: Sintonia do $N_{C}$ para saída C4D. .......................................................... 94

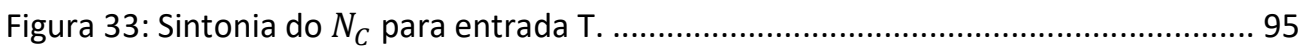

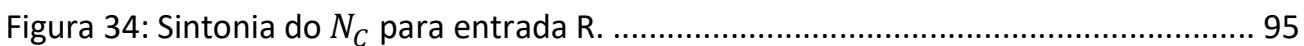

Figura 35: Sintonia do $Q_{Y}$ para saída C3B. ...................................................... 96

Figura 36: Sintonia do $Q_{Y}$ para saída C4D. ....................................................... 96

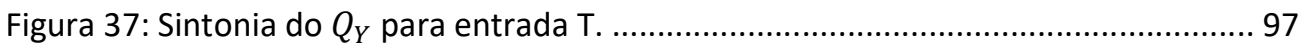

Figura 38: Sintonia do $Q_{Y}$ para entrada R. ....................................................... 97

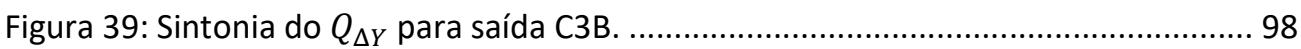

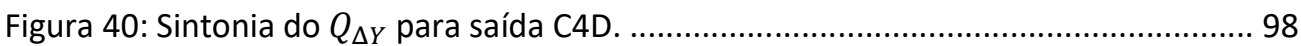

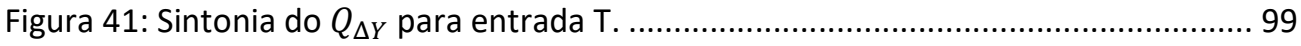

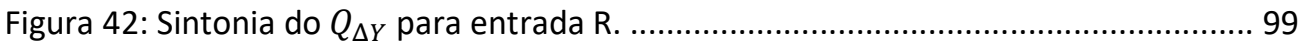

Figura 43: Sintonia do $N_{C}$ para saída C3B. .................................................... 100

Figura 44: Sintonia do $N_{C}$ para saída C4D. ...................................................... 100

Figura 45: Sintonia do $N_{C}$ para entrada T...................................................... 101

Figura 46: Sintonia do $N_{C}$ para entrada R. ..................................................... 101

Figura 47: Sintonia do $Q_{Y}$ para saída C3B. ...................................................... 102

Figura 48: Sintonia do $Q_{Y}$ para saída C4D. ..................................................... 102

Figura 49: Sintonia do $Q_{Y}$ para entrada T. ...................................................... 103

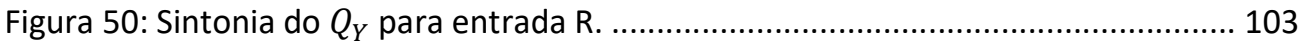

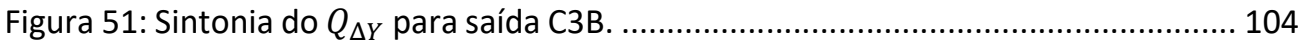

Figura 52: Sintonia do $Q_{\Delta Y}$ para saída C4D. ..................................................... 104

Figura 53: Sintonia do $Q_{\Delta Y}$ para entrada T. ........................................................ 105

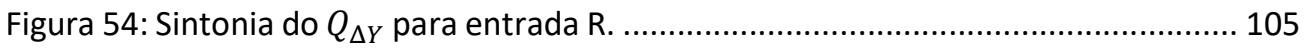

Figura 55: Comparação entre as variáveis estimadas e a trajetória de referência com H-NMPC 106

Figura 56: Entradas T e R calculadas para mudança de trajetória das entradas do H-NMPC. 107

Figura 57: Comparação entre as variáveis controladas e a trajetória de referência com 
NN-NMPC.

Figura 58: Entradas T e R calculadas para mudança de trajetória das entradas do

NN-NMPC.

Figura 59: Comparação entre as variáveis controladas e a trajetória de referência

com controle regulatório.

Figura 60: Entradas T e R calculadas para mudança de trajetória das entradas com

controle regulatório.

Figura 61: Soma dos erros quadráticos dos controladores H-NMPC, NN-NMPC e PID

para teste de rastreamento de trajetória de referência.

Figura 62: Efeito nas saídas causado pelo distúrbio aplicado na vazão de alimentação com H-NMPC.

Figura 63: Entradas calculadas para rejeição de distúrbio na vazão da alimentacao com H-NMPC.

Figura 64: Efeito nas saídas causado pelo distúrbio aplicado na vazão de alimentação com NN-NMPC.

Figura 65: Entradas calculadas para rejeição de distúrbio na vazão da alimentação com H-NMPC.

Figura 66: Efeito nas saídas causado pelo distúrbio aplicado na vazão de alimentação com controlador PID.

Figura 67: Entradas calculadas para rejeição de distúrbio na vazão da alimentação com controlador PID.

Figura 68: Soma dos erros quadráticos dos controladores H-NMPC, NN-NMPC e PID para teste de distúrbio da vazão da alimentação.

Figura 69: Efeito nas saídas causado pelo distúrbio aplicado na composição

da alimentação com H-NMPC.

Figura 70: Entradas calculadas para rejeição de distúrbio na composição da alimentação com H-NMPC.

Figura 71: Efeito nas saídas causado pelo distúrbio aplicado na composição de alimentação com NN-NMPC.

Figura 72: Entradas calculadas para rejeição de distúrbio na composição da alimentação com NN-NMPC.

Figura 73: Efeito nas saídas causado pelo distúrbio aplicado na composição de alimentação com controlador PID.

Figura 74: Entradas calculadas para rejeição de distúrbio na composição da alimentação com controlador PID. 
Figura 75: Soma dos erros quadráticos dos controladores H-NMPC, NN-NMPC e PID para teste de disturbio da composição da alimentação. 


\section{Lista de Tabelas}

Tabela 1: Composição típica do gás natural no Brasil distribuído por estados (Fonte:

VAZ et al., 2009).

Tabela 2: Especificações do GN conforme Resolução ANP No16, de 17.6.2008.

Tabela 3: Resumo dos trabalhos encontrados na literatura em que o modelo de

Hammerstein foi utilizado para representar uma coluna de destilação.

Tabela 4: Resumo dos trabalhos encontrados na literatura em que o modelo de Redes

Neurais foi utilizado para representar uma coluna de destilação.

Tabela 5: Estrutura do controle regulatório.

Tabela 6: Estrutura dos controladores supervisórios da estratégia de controle NMPC e

de controle PID.

Tabela 7: Valores de referência das variáveis controladas para teste de rastreamento

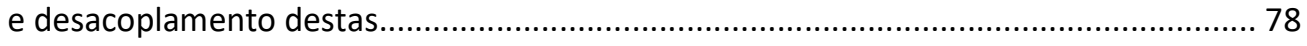

Tabela 8: Coeficientes das equações polinomiais $3 \times 4$ para C3B e C4D. ............................. 83

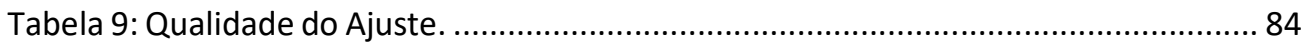

Tabela 10: Parâmetros estimados para o modelo linear. ………….................................. 86

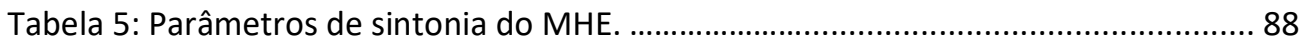

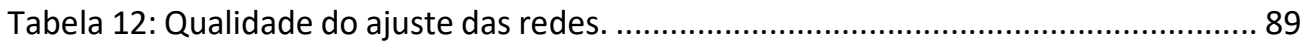

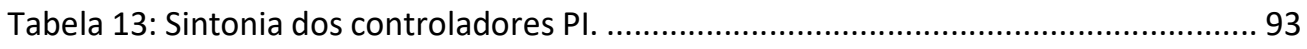

Tabela 6: Resumo dos resultados qualitativos dos testes. ........................................... 122 


\section{Abreviações}

\begin{tabular}{|c|c|c|}
\hline & ANP & Agência Nacional do Petróleo \\
\hline & CARIMA & Modelo controlado auto-regressivo com média móvel incremental \\
\hline & DCS & Sistema de Controle Distribuído \\
\hline & GN & Gás Natural \\
\hline & GPC & Controlador Preditivo Generalizado \\
\hline & H-NMPC & Modelo Preditivo baseado em Modelo Não-linear de Hammerstein \\
\hline & MAC & Controle por Modelo Algorítmico \\
\hline & MHE & Estimador de Horizonte Móvel \\
\hline & MPC & Modelo Preditivo baseado em Modelo \\
\hline & $\mathrm{NN}$ & Redes Neurais \\
\hline & NN-NMPC & Modelo Preditivo baseado em Modelo Não-linear de Redes Neurais \\
\hline & NMPC & Modelo Preditivo baseado em Modelo Não-linear \\
\hline & QDMC & Controlador Quadrático por Matriz Dinâmica \\
\hline & RTO & Otimização em Tempo Real \\
\hline & SSE & Soma do Erro Quadrático \\
\hline & UPGN & Unidade de Processamento de Gás Natural \\
\hline
\end{tabular}




\section{Símbolos}

C1 Metano

C2 Compostos de dois carbonos (ex. etano)

C3 Compostos de três carbonos (ex. propano)

C4 Compostos de quatro carbonos (ex. butano)

C5 Compostos de cinco carbonos (ex. pentano)

C6 Compostos de seis carbonos (ex. hexano)

C3+ Compostos de três carbonos ou mais

C4+ Compostos de quatro carbonos ou mais

C5+ Compostos de cinco carbonos ou mais

C6+ Compostos de seis carbonos ou mais

C3B Fração molar de compostos de três carbonos no produto de fundo

C4D Fração molar de compostos de quatro carbonos no produto de topo

F Vazão da alimentação

R Vazão molar de refluxo

L V Vazão de refluxo (líquido)

V Vazão de refervimento

D Vazão de destilado

B Vazão do produto de fundo

AR Vazão do fluido de refrigeração

$i \quad$ Instante de amostragem

j Instante de amostragem

$k \quad$ Instante de amostragem

$t \quad$ Tempo

$\Delta t \quad$ Período de amostragem, 
$t_{s} \quad$ Tempo de assentamento

$u \quad$ Ação de controle como entrada do controlador

$u^{\text {min }}$ Valor mínimo da ação de controle

$u^{\max }$ Valor máximo da ação de controle

$\Delta u \quad$ Variação da ação de controle como entrada do controlador

$\Delta u^{\text {min }}$ Valor mínimo da variação da ação de controle

$\Delta u^{\text {max }}$ Valor máximo da variação da ação de controle

$x \quad$ Variáveis de estado

$v \quad$ Distúrbio na entrada do sistema

w Ruído de medição

y Resposta predita

$y^{\text {min }}$ Valor mínimo da saída

$y^{\max }$ Valor máximo da saída

$N_{c} \quad$ Horizonte de controle

$N_{p} \quad$ Horizonte de predição

$N_{m} \quad$ Horizonte do modelo

A Matriz referente aos estados do modelo em espaço de estado

$B \quad$ Matriz referente às entradas do modelo em espaço de estado

$B_{v} \quad$ Matriz referente aos distúrbios na entrada do sistema em espaço de estado

$B_{\text {mod }}$ Matriz modificada referente às entradas do modelo em espaço de estado

C Matriz referente às saídas do modelo em espaço de estado

D Matriz de ligação entre entrada e saída

J Função objetivo do MPC

$J_{M H E} \quad$ Função objetivo do MHE

$\gamma \quad$ Vetor dos resíduos referente ao erro do modelo do MHE 
$\gamma_{\mathrm{MHE}}$ Vetor das variáveis de decisão do MHE

W Vetor de ruídos no horizonte de predição

$X \quad$ Vetor de estados no horizonte de predição

$X^{\text {min }}$ Vetor de valores mínimos dos estados no horizonte de predição

$X^{\max }$ Vetor de valores máximos dos estados no horizonte de predição

Y Vetor de saídas no horizonte de predição

$Y^{\min }$ Vetor de valores mínimos das respostas no horizonte de predição

$Y^{\max }$ Vetor de valores máximos das respostas no horizonte de predição

$Y^{S P} \quad$ Vetor das trajetórias de referência das respostas no horizonte de predição

U Vetor das ações de controle no horizonte de controle

$U^{\text {min }}$ Vetor de valores mínimos das ações de controle no horizonte de controle

$U^{\max }$ Vetor de valores mínimos das ações de controle no horizonte de controle

$\Delta U \quad$ Vetor das variações das ações de controle no horizonte de controle

$\Delta U^{\text {min }}$ Vetor de valores mínimos das variações das ações de controle no horizonte de controle

$\Delta U^{\max }$ Vetor de valores mínimos das variações das das ações de controle no horizonte de controle

$Q_{Y} \quad$ Matriz diagonal positiva semi-definida de ponderação do erro entre a variável de referência e a variável de saída predita

$Q_{\Delta U} \quad$ matriz diagonal positiva semi-definida de ponderação em relação à variação das variáveis manipuladas

m Número de entradas

$q \quad$ Número de saídas

n Número de estados

$m_{d} \quad$ Número de distúrbios na entradas

$f(\cdot) \quad$ Função não-linear que representa a entrada do NMPC 
$g(\cdot) \quad$ Função não-linear que representa a saída do NMPC

$\phi \quad$ Vetor das ações de controle modificadas

$\Phi_{\text {mod }}$ Vetor das ações de controle modificadas para um horizonte de controle

Símbolos para Redes Neurais

IW Matriz de pesos da entrada da rede

LW Vetor de pesos da camada escondida $\in \mathbb{R}^{n \times 1}$,

LB Vetor bias da camada escondida $\in \mathbb{R}^{n \times 1}$,

OB Escalar bias da saída,

$g$ Função de ativação da camada escondida,

f $\quad$ Função de ativação da saída.

n Número de neurônios,

$m \quad$ Número de entradas do sistema (u),

z $\quad$ Potencial de ativação

w Ponderação dos valores de entrada

$\Theta \quad$ Limite de ativação ou bias do neurônio

u Entrada da rede

y Saída da rede 


\section{1.}

\section{Introdução}

De acordo com o último relatório produzido pela Confederação Nacional da Indústria no ano de 2018, a indústria de gás natural encontra-se em um momento de grande transformação no mundo inteiro. A exploração de novas fontes energéticas vem aumentando a competitividade da indústria do gás e contribuindo para a queda do preço, um dos mais elevados do mundo. Assim, aumentar a eficiência do mercado de gás natural significa fortalecer a competitividade do segmento (CNI, 2018).

A destilação representa aproximadamente $80 \%$ do custo energético em uma Unidade de Processamento de Gás Natural (UPGN). Por isso, se faz necessário aumentar a sua eficiência de produção, garantindo a pureza dos produtos, a recuperação de cargas recicladas e a minimização do consumo energético do processo (NORMEY-RICO, 2005).

Segundo Ito (2014), o controle das composições dos produtos de uma coluna de destilação apresenta inúmeros desafios em aplicações industriais, como análise em linha com baixas frequências, instrumentação de alto custo e frequente manutenção, o que dificulta o controle em tempo real. Assim, o desenvolvimento de um modelo adequado para a predição indireta de variáveis de difícil medição, na frequência desejada, possibilita as aplicações de controle avançado.

É economicamente importante a escolha da técnica de controle adequada a ser implementada nas colunas de destilação, devido ao efeito do controle na qualidade dos produtos, na taxa de produção, no consumo de energia (HUANG e RIGGS, 2000), no aumento da capacidade e da segurança do processo (ABDULLAH et al., 2007). Controladores monovariáveis usualmente empregados na indústria frequentemente apresentam grande dificuldade em manter os produtos dentro da especificação, pois são geralmente baseados em teorias lineares, causando baixo desempenho frente às não-linearidades do processo. Logo, a necessidade de melhores desempenhos das plantas exige melhores estratégias de controle (WAHID, 2016).

Os tipos de controladores utilizados industrialmente variam de acordo com a natureza da operação e com o desempenho exigido para a rotina de controle do processo. Colunas de destilação possuem características particularmente desafiadoras sob o ponto de vista de controle, como não-linearidades, grandes 
constantes de tempo, restrições de variáveis e inversão do sinal de ganho estático. As colunas de destilação exibem comportamento dinâmico não-linear devido às suas relações de equilíbrio líquido-vapor não-lineares, à alta pureza do produto e às complexas configurações de processamento, como pré-fracionamentos, retiradas laterais de produtos e alimentações múltiplas. Sua dinâmica é influenciada por mudanças de vazão de vapor muito rápidas, mudanças de vazão de líquido moderadamente rápidas, mudanças de temperatura lentas e mudanças de composição muito lentas (ABDULLAH et al., 2007).

Como as medidas de composição frequentemente possuem atrasos e dados esparsos, os sistemas de controle convencionais não são capazes de controlar a composição diretamente e possuem dificuldade em manter os produtos dentro das especificações. Contudo, controladores baseados em modelo possuem a habilidade de prever a composição através do modelo interno do processo, além de ser capaz de lidar com restrições, como restrição hidráulica, restrição de separação, restrição de transferência de calor, restrição de pressão e restrição de temperatura (ABDULLAH et al., 2007).

Dentre as classes de controladores avançados, destaca-se o Controle Preditivo baseado em Modelo (MPC - do inglês, Model Predictive Control), pela facilidade de modelos lineares serem desenvolvidos e interpretados (SEBORG et al., 2010). O MPC é bem estabelecido na indústria para controle de processos multivariáveis com restrições. Porém, este descreve o comportamento do sistema por modelos dinâmicos lineares, sendo inadequado para processos altamente não-lineares que possuem uma ampla faixa de regimes operacionais. Essa deficiência, combinada com demandas cada vez mais exigentes sobre a produção e a qualidade do produto, vem estimulando o desenvolvimento do Controle Preditivo baseado em Modelo Não-linear (NMPC - do inglês, Nonlinear Model Predictive Control), o qual é capaz de descrever o sistema por modelos dinâmicos não-lineares. (HENSON, 1998).

Apesar de claras vantagens, a aplicação do NMPC na indústria de processos ainda é limitada. Nos últimos anos, o crescente interesse da indústria e da área científica, aliada ao desenvolvimento tecnológico, permitiu o estudo e a implementação de diversas técnicas de controle de processos não-lineares como o NMPC.

Segundo Abdulhah et al. (2007), o modelo de redes neurais é a estrutura mais popular para o desenvolvimento de modelos empíricos para colunas de destilação, devido a facilidade da modelagem do comportamento dinâmico do sistema. Modelo do tipo Hammerstein é outro modelo empírico comumente 
empregado para prever o comportamento de sistemas não-lineares como colunas de destilação.

Ademais, variações no modelo Hammerstein estão presentes na literatura. O modelo de Hammerstein modificado proposto por Miyoshi (2016) propõe uma estrutura com uma mudança de variável de forma a obter uma solução analítica do problema de predição não-linear futura com maior rapidez computacional dos cálculos matriciais. Esta técnica foi aplicada para o controle de um sistema de Reator CSTR de Van de Vusse e para um processo de primeira ordem (Miyoshi, 2016) e de um sistema gás-lift (SALLES e NUNES, 2017). Essas aplicações foram para controle univariável, logo, a aplicação deste modelo de Hammerstein modificado para controle multivariável de uma coluna de destilação é uma novidade na literatura.

\section{1.}

Objetivo do trabalho

Face ao exposto, este trabalho tem como objetivos:

- Realizar a simulação dinâmica da coluna de destilação despropanizadora,

- Coletar dados simulados do processo para identificação do sistema,

- A partir dos dados de simulação, efetuar a modelagem do sistema utilizando as duas técnicas empíricas:

- Hammerstein,

- Redes Neurais.

- Comparar as três estratégias de controle: NMPC com modelo de Hammerstein (H-NMPC), NMPC com modelo de Redes Neurais (NNNMPC) e controle PID tradicional.

\section{2 .}

Organização do trabalho

Este trabalho está subdividido em sete capítulos.

No Capítulo 1, é descrita a introdução e a motivação do trabalho, apresentando nas seções os objetivos e a organização deste.

No Capítulo 2, é apresentada uma revisão bibliográfica abordando: o funcionamento de uma Unidade de Processamento de Gás Natural, a arquitetura 
de controle, o Controle Preditivo baseado em Modelo e o Controle Preditivo baseado em Modelo Não-Linear.

O capítulo 3 descreve a metodologia para a simulação estática e dinâmica da coluna despropanizadora em Aspen Plus, a comunicação entre os softwares Aspen e Matlab para a implementação dos controladores NMPC e a obtenção dos dados para modelagem do sistema. Este capítulo também apresenta a metodologia para a modelagem e identificação dos parâmetros dos modelos Hammerstein e Redes Neurais, a metodologia para a sintonia dos controladores NMPC e a metodologia para os testes de performance dos controladores. $O$ Capítulo 4 apresenta os resultados obtidos.

O Capítulo 5 apresenta as conclusões do trabalho.

O Capítulo 6 apresenta possíveis trabalhos futuros.

Por fim, apresenta-se as referências bibliográficas no Capítulo 7. 


\section{2.}

\section{Revisão bibliográfica}

Neste capítulo, é realizada uma revisão bibliográfica sobre o processamento de gás natural a ser estudado, arquitetura de controle e controle preditivo baseado em modelo linear e não-linear.

\section{1.}

\section{Unidade de Processamento de Gás Natural (UPGN)}

O gás natural (GN) é uma mistura de hidrocarbonetos obtidos através das reservas naturais de petróleo, a qual permanece na fase gasosa nas condições atmosféricas de pressão e temperatura. Os principais hidrocarbonetos constituintes são o metano, o etano, o propano e o butano e os gases inorgânicos nitrogênio, dióxido de carbono e gás sulfídrico (VAZ et al., 2009). As composiçõesvolumétricas do gás natural estão listadas na Tabela 1.

Tabela1: Composição típica do gás natural no Brasil distribuído por estados (Fonte: VAZ et al., 2009).

\begin{tabular}{cccccccc}
\hline Composição & CE/RN & SE/AL & BA & ES & RJ & SP & AM \\
\hline C1 \% vol. & 74,53 & 81,32 & 81,14 & 88,16 & 79,69 & 87,88 & 68,88 \\
C2 \% vol. & 10,40 & 8,94 & 11,15 & 4,80 & 9,89 & 6,27 & 12,20 \\
C3 \% vol. & 5,43 & 3,26 & 3,06 & 2,75 & 5,90 & 2,86 & 5,19 \\
C4 \% vol. & 2,81 & 1,84 & 1,39 & 1,55 & 2,13 & 1,16 & 1,80 \\
C5 \% vol. & 1,30 & 0,74 & 0,72 & 0,44 & 0,77 & 0,27 & 0,43 \\
C6+ \% vol. & 1,40 & 0,42 & 0,30 & 0,44 & 0,44 & 0,07 & 0,18 \\
$\mathrm{~N}_{2} \%$ vol. & 1,39 & 1,51 & 1,43 & 1,62 & 0,80 & 1,16 & 11,12 \\
$\mathrm{CO}_{2} \%$ vol. & 2,74 & 1,97 & 0,81 & 0,24 & 0,50 & 0,23 & 0,20 \\
$\mathrm{H}_{2} \mathrm{~S} \mathrm{mg} / \mathrm{m}^{3}$ & 1,50 & 7,50 & 7,60 & 7,50 & 6,70 & traços & --- \\
\hline
\end{tabular}

O gás natural costuma ser produzido nos campos de óleo e gás na forma de gás associado ao petróleo e transportado através de gasodutos até as Unidades de Processamento de Gás Natural (UPGN). Nessas unidades, o gás natural é processado e é separado da fase líquida (água e hidrocarbonetos líquidos). O processamento do gás consiste em uma sucessão de operações unitárias, as quais purificam, desidratam, resfriam e fracionam os componentes em seus produtos. O projeto de uma UPGN é feito de acordo com as 
características do gás natural, que varia em sua composição a cada região de extração (BARALDI, 2015). A Figura 1 exemplifica um esquema para representar o fracionamento do gás natural.

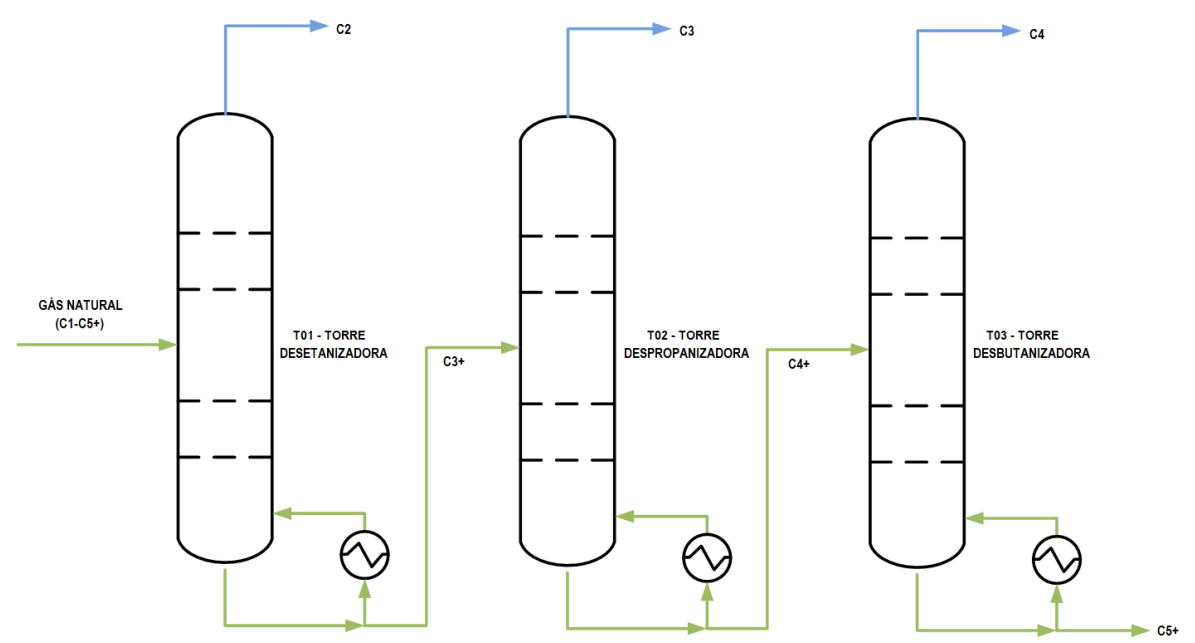

Figura 1: Esquema simplificado das colunas de destilação de uma UPGN.

Fonte?

Nesta configuração apresentada na Figura 1, a UPGN é composta pelas colunas de destilação desetanisadora, despropanizadora e desbutanizadora (HUANG e RIGGS, 2000). A alimentação da desetanizadora é uma mistura de hidrocarbonetos que vai das frações de metano até a gasolina natural (C1-C5+) e esta tem majoritariamente como produto de topo metano e etano (C1-C2). As frações com três ou mais carbonos $(\mathrm{C} 3+)$ restantes seguem para a coluna despropanizadora. Nesta coluna, as frações de três carbonos (C3) são destiladas no topo da coluna e as frações com quatro ou mais carbonos $(\mathrm{C} 4+)$ restantes seguem para a coluna desbutanizadora. Por fim, esta coluna tem como produto de topo as frações de quatro carbonos (C4) e como produto de fundo as frações com cinco ou mais carbonos (C5+).

Múltiplos objetivos de controle precisam ser atendidos para a UPGN. Segundo CAMPOS (2006), é necessário o controle da pressão de cada coluna e dos níveis de estoques de líquidos nos condensadores e nos refervedores, com o objetivo de atingir a segurança na operação. Na sequência, as especificações de impureza dos produtos propano e butano devem ser mantidas diante de vários distúrbios, como variação na vazão e composição da alimentação.

De acordo com HUANG e RIGGS (2000), somente a fração molar dos componentes de dois cabonos (C2) e de três ou mais carbonos (C3+) no produto 
de fundo devem ser controladas na coluna desetanizadora. Dessa forma, a impureza do produto de topo não requer um controle rigoroso. Para o controle da coluna desbutanizadora, a mesma filosofia pode ser aplicada de forma que apenas a impureza do produto de topo (C5+ em C4) é controlada. Portanto, essas duas colunas exigem apenas um único controle de composição (single-ended). Contudo, a coluna despropanizadora, por ser o gargalo do sistema, precisa de controle de impureza rigoroso em ambos os produtos (HUANG e RIGGS, 2000).

Este trabalho compreende o estudo de controle somente da coluna despropanizadora. A descrição desta com dados mais detalhados se encontra na seção 3.1.1.

\subsection{1.}

\section{Especificação dos produtos}

O gás natural é processado para atender às diversas especificações requeridas pelo mercado, permitindo a obtenção de produtos com maior valor agregado. As especificações exigidas para o gás natural são referentes à legislação e às especificações técnicas de projeto, para que não ocorram danos às instalações de produção e aos gasodutos.

As especificações para a comercialização do gás natural no Brasil são estabelecidas pela ANP (Agência Nacional do Petróleo, Gás Natural e Biocombustíveis). De acordo com a Resolução ANP №16, de 17.6.2008, com excessão da região Norte, o destilado propano não deve conter teor de moléculas com quatro ou mais carbonos (C4+) acima de $3 \%$ e o produto de fundo butano não deve conter teor de moléculas de três ou menos carbonos (C3-) acima de 6\%, como observado na Tabela 2. 
Tabela 2: Especificações do GN conforme Resolução ANP No16, de 17.6.2008.

\begin{tabular}{lcccc}
\hline \multirow{2}{*}{ Características } & \multirow{2}{*}{ Unidade } & \multicolumn{3}{c}{ Limite por região } \\
\cline { 3 - 5 } & & $\mathbf{N}$ & NE & CO, S e SU \\
\hline Poder calorífico superior & $\mathrm{kJ} / \mathrm{m}^{3}$ & 34 a $38.4 \mathrm{k}$ & 35 a $43 \mathrm{k}$ & 35 a $43 \mathrm{k}$ \\
Índice de Wobbe & $\mathrm{kJ} / \mathrm{m}^{3}$ & 40.5 a $45 \mathrm{k}$ & 46.5 a $53.5 \mathrm{k}$ & 46.5 a $53.5 \mathrm{k}$ \\
Metano, mín. & $\% \mathrm{~mol}$ & 68 & 85 & 85 \\
Etano, máx. & $\% \mathrm{~mol}$ & 12 & 12 & 12 \\
Propano, máx. & $\% \mathrm{~mol}$ & 3 & 6 & 6 \\
Butanos e mais pesados, máx & $\% \mathrm{~mol}$ & 1,5 & 3 & 3 \\
Oxigênio, máx & $\% \mathrm{~mol}$ & 0,8 & 0,5 & 0,5 \\
Inertes ( $\left.\mathrm{N}_{2}+\mathrm{CO}_{2}\right)$, máx & $\% \mathrm{~mol}$ & 18 & 8 & 6 \\
CO 2 máx & $\% \mathrm{~mol}$ & 3 & 3 & 3 \\
Enxofre Total, máx & $\mathrm{mg} / \mathrm{Nm}^{3}$ & 70 & 70 & 70 \\
Gás Sulfídrico $\left(\mathrm{H}_{2} \mathrm{~S}\right)$, máx & $\mathrm{mg} / \mathrm{Nm}^{3}$ & 10 & 13 & 10 \\
\hline
\end{tabular}

As especificações dos produtos são definidas pelas demandas dos processos a jusante, pelo cliente e/ou pela região do país. Os produtos devem satisfazer certas normas de qualidade e podem exigir uma alimentação ao processo com qualidade predeterminada.

Um dos problemas mais comuns no controle de despropanizadoras é encontrar as purezas do produto que maximizam o lucro. Em muitas colunas, a pureza de um produto pode ser fixada por uma especificação máxima de impureza, mas o outro produto pode não ter pureza definida. Como o produto propano é mais valioso do que o butano, a vazão de destilados deve ser maximizada de modo que a parcela de butano nessa vazão seja a maior possível, até o limite máximo de impureza. Isto pode ser conseguido minimizando a concentração de propano que é perdido no fundo (C3B). Porém, reduzir o C3B requer um aumento na entrada de calor do refervedor, o que aumenta o custo de energia (Luyben, 2006).

Exceder às especificações do produto ou produzir um produto de melhor qualidade do que é necessário é conhecido como produto Giveaway - doado ou desperdiçado, em inglês. Quando isso ocorre, tanto o produto quanto a energia são desperdiçados, reduzindo a margem de lucro da instalação. Assim, as medições das composições são feitas para garantir que os produtos de um sistema de destilação estejam de acordo com a especificação (SKOGESTAD, 2007). 


\subsection{2. \\ Variáveis operacionais}

Para se garantir a especificação dos produtos, as seguintes variáveis devem ser observadas:

1) Vazão da alimentação $F$

2) Composição da alimentação

3) Temperatura da alimentação

4) Pressão da coluna

5) Temperatura da coluna

6) Nível do condensador

7) Nível do refervedor

8) Vazão de refluxo $R$

9) Vazão de refervimento $V$

10) Vazão do destilado $D$

11) Vazão do produto de fundo $B$

Os distúrbios mais recorrentes em colunas de destilação são mudanças na composição, vazão ou entalpia da alimentação, os quais repercutem na variabilidade das impurezas nos produtos. A vazão e a composição da alimentação de uma despropanizadora são difíceis de serem manipuladas, uma vez que estas dependem da saída de produtos da desetanizadora, sendo necessário um bom controle de forma a rejeitar estes distúrbios. A temperatura da alimentação é controlada e regulada, mas não é possível controlar sua entalpia, uma vez que esta depende de sua composição (Rech, 2013).

O controle da pressão é fundamental para a operação da coluna, pois a sua variação implica na variação das constantes de equilíbrio dos componentes ao longo da coluna, dificultando a manutenção da composição dos produtos dentro das especificações desejadas. O controle dos níveis dos condensadores e refervedores garante que não existirá um acúmulo ou falta de massa no sistema, evitando problemas de cavitação nas bombas, associados à inundação ou esvaziamento dos vasos da coluna (CAMPOS, 2006).

A temperatura da coluna é manipulada de forma a controlar indiretamente a composição dos produtos. Como a pressão da coluna é mantida constante, a composição dos produtos está diretamente associada ao perfil de temperatura da coluna, dada uma certa composição da alimentação, podendo ser feito um 
controle indireto inferencial das composições (CAMPOS, 2006). Além do fato de sensores de temperatura serem baratos e confiáveis, a medição da temperatura em locais estratégicos da coluna é capaz de fornecer informações bastante precisas sobre as concentrações dos componentes. Alguns dos critérios para escolher o local do sensor de temperatura são por inclinação (maior variação de temperatura num prato) e sensibilidade (maior variação frente da temperatura frente a variações nas variáveis manipuladas num prato) (LUYBEN, 2006).

A vazão de refluxo e de vapor são as variáveis manipuladas mais importantes do processo, controlam o perfil de temperatura da coluna estando diretamente relacionadas ao nível de fracionamento desejado. Um aumento na vazão de refluxo implica na melhora na qualidade do destilado e um aumento na vazão de refervimento implica na melhora do produto de fundo. Deve-se estabelecer limites inferiores para estas variáveis, que restringem a qualidade dos produtos e as capacidades do condensador e do refervedor.

As vazões dos produtos de topo e de fundo são comumente variáveis manipuladas para controlar os níveis do condensador e refervedor, respectivamente, mas pode variar dependendo da estrutura de controle escolhida. Ambas podem ser controladas de forma a aumentar ou diminuir as vazões de acordo com a necessidade do cliente ou do processo à jusante.

Esta análise se faz necessária antes de montar uma estrutura de controle, que está descrita na seção a seguir.

\subsection{3.}

\section{Estruturas de controle para coluna de destilação}

A seleção da estratégia a ser adotada é nos controles de colunas de destilação requer um profundo conhecimento do processo e da teoria de controle. Não existe uma estratégia única que seja adequada a todo tipo de coluna. Em geral, a melhor estratégia é aquela que resulta em um equilíbrio adequado entre redução do acoplamento (entre as malhas de controle de composição), aumento da capacidade de rejeitar distúrbios, redução da não-linearidade do processo e simplicidade do sistema de controle (SKOGESTAD, 2007). 


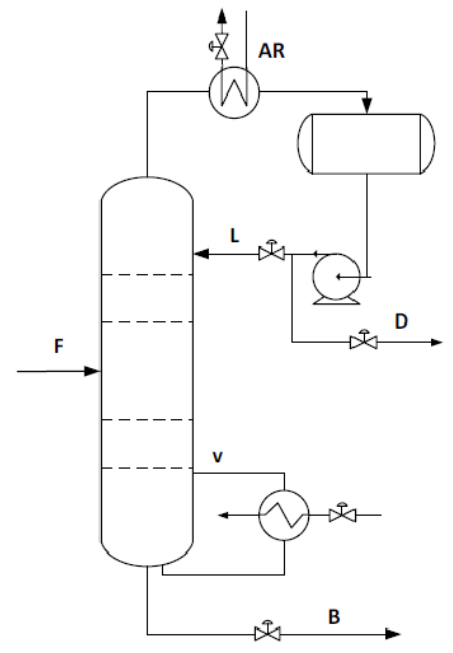

Figura 2: Graus de liberdade de uma coluna de destilação.

$\mathrm{Na}$ escolha correta de uma estrutura de controle, é necessário analisar os graus de liberdade da planta, definir os objetivos de controle e as variáveis controladas e manipuladas. Os graus de liberdade do sistema estão associados às válvulas de controle, indicando as variáveis manipuladas. Para a coluna de destilação da Figura 2, existem 5 graus de liberdade, referentes à:

- D: vazão do produto de topo;

- $\quad$ B: vazão do produto de fundo;

- $\quad$ AR: vazão do fluido de refrigeração;

- V: vazão de refervimento;

- $\quad$ L: vazão de refluxo.

A vazão de alimentação é indicada por $F$.

Segundo Campos (2006), sistemas bem projetados possuem a mesma quantidade de variáveis manipuladas e controladas. Portanto, podem-se escolher até cinco variáveis do processo a serem controladas para esta coluna.

O termo "estrutura" de controle para as colunas de destilação refere-se às duas combinações das quatro vazões $L, V, D$ e $B$ que permanecem como graus de liberdade depois de os circuitos de nível terem sido fechados. As estruturas são representadas por siglas em que a primeira letra representa a variável manipulada para controle da composição do destilado e a segunda letra representa a variável que é manipulada de controle da composição do produto de fundo. Tradicionalmente, a vazão de refluxo (L), a vazão de destilado (D) ou a razão de refluxo (L/D) é usada para controle da composição do produto de topo, enquanto a carga térmica do refervedor $\left(Q_{R}\right)$ ou a vazão de refervimento $(V)$, a 
vazão de produto da base (B) ou a razão entre elas (V/B) é manipulada para controle da composição do produto de fundo (SKOGESTAD, 2007).

Ao usar D e B para controlar o nível do condensador e do refervedor, respectivamente, $L$ e $V$ permanecem como graus de liberdade - esta estrutura é, portanto, chamada de estrutura LV. A configuração LV é a escolha mais comum ou "convencional" e está representada na Figura 3.

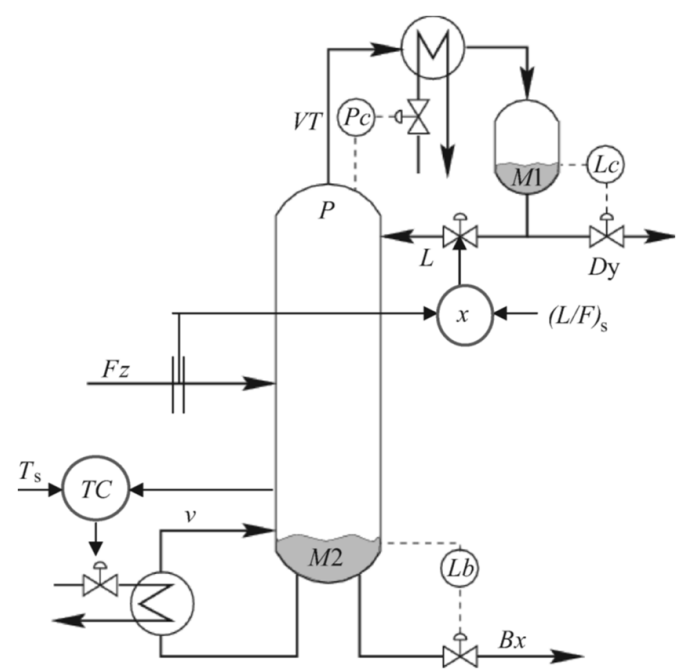

Figura 3: Coluna de destilação controlada com a estrutura LV, com circuito de temperatura em V e um controle feedforward em L/F. Fonte: Skogestad, 2007.

Segundo Huang e Riggs (2000), a estrutura LV é uma das melhores estruturas de controle para colunas despropanizadoras, exceto quando são de alta pureza (menos de 1\% de impureza).

\section{2.}

\section{Arquitetura de controle}

Uma solução comum para gerenciar sistemas complexos e distribuir a tomada de decisões é uma abordagem hierárquica para decompor um problema incontrolável em uma cascata de problemas solucionáveis interconectados. A construção de modelos de sucesso em uma hierarquia inclui determinar o que é relevante para as tomadas de decisão específicas e omitir os detalhes irrelevantes (DARBY et al., 2011).

Conforme apresentadas na Figura 4, as camadas de um sistema de controle podem incluir:

- Planejamento da produção (Planning); 
- Programação da produção (Scheduling);

- Otimização em tempo real (RTO);

- Controle supervisório/preditivo;

- Sistema de controle distribuído responsável pelo controle regulatório.

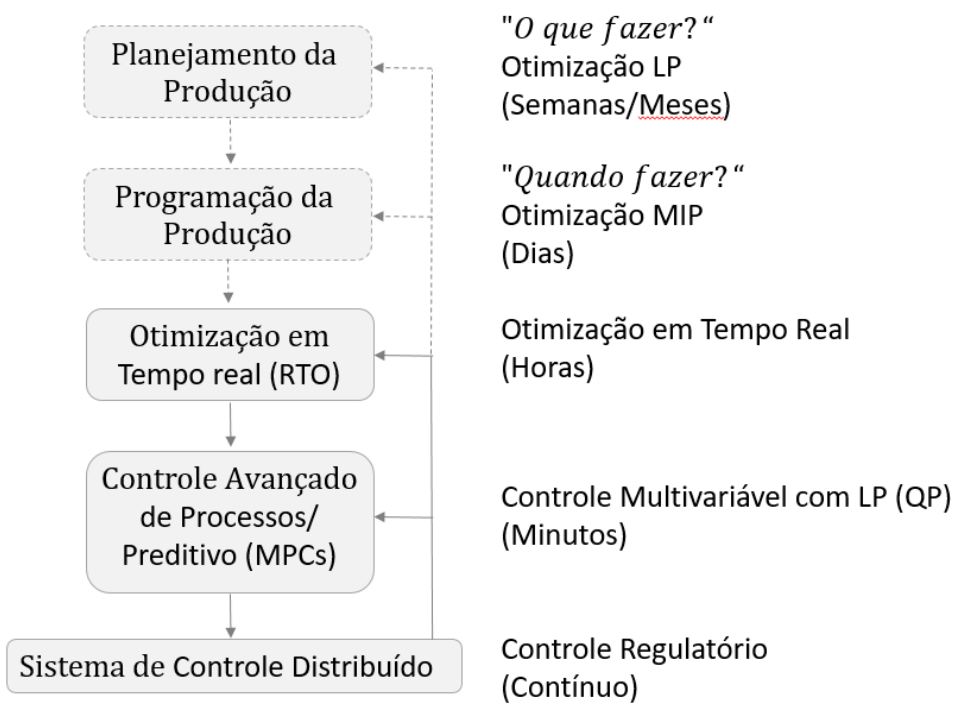

Figura 4: Hierarquia de controle típica em uma planta de processos químicos.

Adaptado de DARBY et al., (2011)

O planejamento se preocupa com o que e como fazer, baseado em uma função geral de objetivo de lucro da planta e conhecimentos prévios, para decidir quais materiais comprar, quais produtos fabricar e quanto de cada produto produzir (DARBY et al., 2011). Segundo Shobrys e White (2002), o planejamento estratégico é um processo de longo prazo que prevê mudanças desejadas nos negócios atuais, as quais podem afetar o acesso a matérias-primas, a capacidade de produção ou a distribuição. Já o planejamento tático ocorre em dois níveis: define metas para o desempenho operacional, e coordena atividades em vendas, gestão de materiais, fabricação e distribuição.

A programação da produção define quando fazer e a quantidade de atividades necessárias para atender aos objetivos da empresa. As principais variáveis de decisão são a hora, o local, a duração e volume do processo. A demanda do produto conduz unidades-chave a operação (SHOBRYS e WHITE, 2002).

A otimização em tempo real (RTO) é implementada onde for economicamente justificado e é tipicamente formulada com base em uma função de lucro da planta. A RTO programa em tempo real as decisões de negócio obtidas 
nas camadas acima através de modelos não-lineares estacionários calibrados que a etapa de planejamento não contempla (DARBY et al., 2011). A camada de otimização recalcula as novas trajetórias de referência a cada hora, enquanto a camada de feedback opera continuamente. As camadas são ligadas pelas variáveis controladas, sendo as trajetórias de referência calculadas pela camada superior e implementadas pela camada inferior (LARSSON e SKOGESTAD, 2000).

A camada de Controle Preditivo baseado em Modelo (MPC) efetua o controle dinâmico minuto-a-minuto da planta e fornece uma certa quantidade de capacidade de otimização, indicando o caminho ótimo a ser percorrido pela camada de controle regulatório até o valor ótimo econômico indicado pela camada de RTO.

A camada mais abaixo é o sistema de controle distribuído (DCS - do inglês, Distributed Control System), responsável pelo controle regulatório tipicamente composto por controladores do tipo Proporcional, Integral e Derivativo (PID) e geralmente executa em uma segunda escala de tempo (sub-segundo a multisegundo).

Cada nível na hierarquia pode afetar diferentes departamentos na organização. O sucesso da RTO requer suporte de toda a organização. Se não for planejado, a RTO (mais do que o MPC) pode criar conflito dentro da organização e levar ao fracasso da aplicação da RTO (Darby et al., 2011). A arquitetura é concebida de tal forma que se houver falha no nível de controle avançado, a operação da planta continua com os últimos pontos de ajuste do PID no DCS (CAMPOS, 2006).

\section{3. \\ Controle Preditivo Baseado em Modelo (MPC)}

O MPC refere-se a uma classe de algoritmos de controle em que um modelo dinâmico do processo é usado para prever e otimizar seu desempenho. As primeiras técnicas de MPC foram desenvolvidas na década de 1970, porque os controladores convencionais univariáveis eram incapazes de satisfazer requisitos de desempenho cada vez mais rigorosos (QIN e BADGWELL, 1997). O objetivo do MPC de primeira geração era resolver problemas típicos de controle com restrições multivariáveis na indústria. No entanto, o MPC não foi introduzido na indústria de processo até os anos 80 (DING et al., 2018). 
Um notável trabalho de revisão de toda a teoria do MPC, desde os MPCs clássicos até teorias de MPCs mais avançadas na literatura foi feito por Ding et al. (2018) e um resumo desta revisão segue abaixo.

Muitos algoritmos para MPC considerados clássicos surgiram nos anos 80 , sendo os mais relevantes o Controlador de Matriz Dinâmica (DMC - do inglês, Dynamic Matrix Control), Controlador por Modelo Algorítimico (MAC - do inglês, Model Algorithmic Control), and Controlador Preditivo Generalizado (GPC - do inglês, Generalized Predictive Control).

O DMC foi desenvolvido nos Estados Unidos pela Shell Oil (Cutler e Ramaker, 1980) e utiliza um modelo de resposta ao degrau, que é relativamente fácil de desenvolver e capaz de descrever sistemas não-lineares.

Em 1986 surgiu o Controlador Quadrático por Matriz Dinâmica (QDMC do inglês Quadratic Dynamic Matrix Control), proposto por Garcia e Morshedi, em que propuseram a solução do controlador DMC através da programação quadrática, introduzindo restrições em relação às variáveis controladas e manipuladas, reduzindo o custo do sistema.

Richalet et al. (1978) estabeleceu o conceito original do MAC no final da década de 1970. O MAC envolve essencialmente um modelo de resposta ao impulso, uma trajetória de referência, um critério de otimalidade e restrições de estado e sinal de controle. O objetivo do MAC é obter uma estratégia de controle ideal para minimizar o critério relevante, que reflete os desvios futuros dentro de um determinado intervalo.

O modelo GPC foi desenvolvido em 1987 por Clarke et al. (1987a, 1987b). O princípio do GPC é gerar uma série de sinais de controle em cada intervalo de amostragem para otimizar o esforço de controle, de forma a rastrear a trajetória de referência com precisão. A idéia central é desenvolver uma estratégia de controle que possa se adaptar às mudanças dinâmicas na taxa de amostragem. O objetivo do GPC é substituir o auto-regulador para resolver problemas de robustez. Este tem a vantagem de auto-sintonizar através da identificação online do modelo, controlando melhor instabilidades e atrasos. A desvantagem é a difícil implementação para sistemas multivariáveis.

Os MPCs clássicos não conseguem atender a requisitos industriais complexos. O objetivo da próxima geração de MPC, desenvolvida no início do milênio, conhecido como MPC aprimorado, era abordar a robustez e a não linearidade dos problemas de controle. São estes o MPC adaptativo, MPC robusto e o NMPC. 
O MPC adaptativo foi desenvolvido de forma a considerar restrições das variáveis de entrada, saída e estado, normalmente aplicado para sistemas lineares. A desvantagem é a dificuldade de garantir a estabilidade e a viabilidade.

A finalidade do controle robusto é projetar controladores que mantenham a estabilidade e o desempenho, apesar das imprecisões ou incertezas do modelo. Essa abordagem pode manter uma estabilidade robusta, simplificando as metas e as descrições de incertezas para solucionar problemas e facilitar o trabalho online. No entanto, as aplicações reais de produção são muito mais complexas, sendo estas conceituais.

Com o aumento contínuo do desempenho e dos requisitos de tempo de computação, foram desenvolvidos MPCs mais avançados, como MPC híbrido, MPC estocástico, MPC distribuído e MPC explícito. O objetivo desta última geração de MPC é diminuir os tempos de cálculo.

\subsection{1. \\ Estratégia do controlador}

O MPC se refere a uma classe de algoritmos avançados que é capaz de prever a resposta futura de um sistema através de um modelo do processo, dadas as medições ou estimativas do estado atual do sistema e uma trajetória de referência. Uma série de entradas de controle é computada a cada instante de amostragem num horizonte de controle, mas apenas a primeira entrada computada é implementada no processo, e então, o algoritmo todo o cálculo é repetido nos horizontes de controle subsequentes (DING et al., 2018).

Segundo Camacho e Alba (2013), a metodologia de todos os controladores pertencentes à família MPC é caracterizada pela seguinte estratégia, representada na Figura 5 e descrita a seguir. 


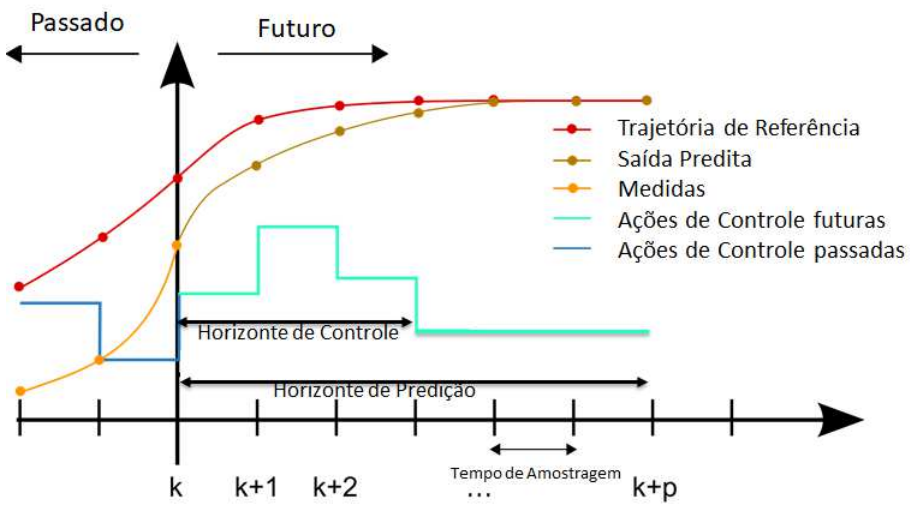

Figura 5: Estratégia do MPC.

1. A predição da variável de saída (y) é realizada para o horizonte de predição $\left(N_{p}\right)$, a cada instante $t$, através de um modelo do processo. Esta predição das saídas é dada por: $y(t+k \mid t)$, em que $k=1,2, \ldots N$, e depende dos valores passados conhecidos até o instante $t$ e das ações de controle futuros $u(t+k \mid t)$, em que $k=0,1, \ldots N-1$.

2. O conjunto de ações de controle futuras é calculada otimizando-se um critério determinado a fim de manter o processo o mais próximo possível da trajetória de referência $w(t+k)$. Este critério geralmente assume a forma de uma função quadrática dos erros entre o sinal de saída predito e a trajetória de referência.

3. O sinal de controle $u(t \mid t)$ é aplicado no processo enquanto as próximas ações de controle são rejeitadas. O próximo instante de amostragem $y(t+$ 1) é conhecido, o passo 1 é repetido com este novo valor e todas as sequências são atualizadas. Assim, as ações $u(t+1 \mid t+1)$ são calculadas usando o conceito de horizonte deslizante ou, do inglês, receding horizon.

Um modelo é, então, usado para prever as saídas futuras da planta, com base nos valores passados e atuais e nas ações de controle futuras calculadas. O modelo escolhido deve ser capaz de capturar a dinâmica do processo, de modo a prever com precisão as saídas futuras, além de ser simples de implementar e compreeder. Como o MPC não é uma técnica única, mas um conjunto de metodologias diferentes, existem muitos tipos de modelos usados em várias formulações, entre estes, estão: resposta ao degrau, resposta ao impulso, funções de transferência, modelo em espaço de estados, modelo Autoregressivo com 
Média Móvel Incremental Controlada (CARIMA - do inglês Controlled Autoregressive Integrating Moving Average), etc.

\subsection{2.}

\section{Formulação do modelo linear em espaço de estado}

Os sistemas MPC são projetados com base em um modelo matemático linear da planta, geralmente por um modelo de espaço de estado. Usando um modelo de espaço de estado, as informações atuais necessárias para prever antecipadamente são representadas pelas variáveis de estado no momento atual (WANG, 2009).

Considerando-se que um modelo linear tenha $m$ entradas $(u), q$ saídas $(y)$, $n$ variáveis de estados $(x)$ e $m_{d}$ distúrbios na entrada $(v)$, o modelo da planta em espaço de estado representado na forma discreta é dado pelas Equações

$$
\begin{aligned}
& x(k+1)=A x(k)+B u(k)+B_{v} v(k), \\
& y(k+1)=C x(k+1),
\end{aligned}
$$

em que

$x \in \mathbb{R}^{n \times 1}, y \in \mathbb{R}^{q \times 1}, u \in \mathbb{R}^{m \times 1}, v \in \mathbb{R}^{m_{d} \times 1}$,

$A \in \mathbb{R}^{n \times n}, B \in \mathbb{R}^{n \times m}, B_{v} \in \mathbb{R}^{n \times m_{d}}, C \in \mathbb{R}^{q \times n}$.

Para a formulação geral de um modelo de espaço de estado, a equação $y(k)=C x(k)+D u(k)$ possui um termo direto do sinal de entrada $u(k)$ para a saída $y(k)$. No entanto, devido ao princípio do controle por horizonte deslizante, onde uma informação atual da planta é necessária para previsão e controle, é assumido implicitamente que a entrada $u(k)$ não pode afetar a saída $y(k)$ ao mesmo tempo. Assim, $D=0$ no modelo da planta (WANG, 2009).

\subsection{3.}

\section{Função objetivo com restrições}

WANG (2009) propõe uma estratégia na qual a função objetivo é minimizada para calcular um conjunto de movimentos ótimos das variáveis manipuladas $(U)$ num horizonte de controle $\left(N_{c}\right)$, de forma a prever as saídas num horizonte de predição $\left(N_{p}\right)$, considerando-se as restrições físicas do sistema a ser 
controlado, é a formulação do Controlador Quadrático por Matriz Dinâmica (QDMC) dada pela Equação (3)

$J=0.5\left(Y^{s p}-Y\right)^{T} Q_{Y}\left(Y^{s p}-Y\right)+0.5 \Delta U^{T} Q_{\Delta U} \Delta U$,

em que $Q_{Y}$ é uma matriz diagonal positiva semi-definida de ponderação do erro entre a referência e a variável de saída predita e $Q_{\Delta U}$ é uma matriz diagonal positiva semi-definida de ponderação da variação das variáveis manipuladas.

A função objetivo está sujeita às seguintes restrições:

$$
\begin{array}{lll}
u^{\text {min }} \leq u(k+i) \leq u^{\max } & \text { (Restrição I) } & \forall i \in \mathbb{Z}, i \in\left[0, N_{\mathrm{c}}-1\right] \\
\Delta u^{\text {min }} \leq \Delta u(k+i) \leq \Delta u^{\text {max }} & \text { (Restrição II) } & \forall i \in \mathbb{Z}, i \in\left[0, N_{\mathrm{c}}-1\right] \\
y^{\text {min }} \leq y(k+j) \leq y^{\max } & \text { (Restrição III) } & \forall \mathrm{j} \in \mathbb{Z}, \mathrm{j} \in\left[1, N_{\mathrm{p}}\right] .
\end{array}
$$

Uma vez formuladas as restrições como parte dos requisitos de projeto, primeiramente é feita a transformação matemática das Restrições I e II em uma desigualdade linear $(a x \leq b)$ de forma a relacioná-la ao problema do MPC original, em que o vetor $U$ é o conjunto das variáveis de decisão de otimização (ou a variável $x$ da desigualdade linear). Em seguida, é feita a transformação matemática da Restrição III em uma desigualdade não-linear $(c(x) \leq 0)$.

Uma vez que o problema de controle preditivo é formulado e resolvido no âmbito do controle de horizonte deslizante, as restrições são levadas em consideração para cada janela de horizonte móvel. Isso permite variar as restrições no início de cada janela de otimização e também fornece os meios para lidar numericamente com o problema de controle restrito (WANG, 2009).

Para atender a Restrição I, dado um sistema MIMO com $m$ entradas, os limites mínimos e máximos do sinal de controle $u$ são dados pelos vetores $u^{\min } \mathrm{e}$ $u^{\max }$, conforme as Equações (7) e (8)

$$
\begin{aligned}
& u^{\min }=\left[\begin{array}{llll}
u_{1}^{\min } & u_{2}^{\min } & \ldots & u_{m}^{\min }
\end{array}\right]^{T} \in \mathbb{R}^{m \times 1} \\
& u^{\max }=\left[\begin{array}{llll}
u_{1}^{\max } & u_{2}^{\max } & \ldots & u_{m}^{\max }
\end{array}\right]^{T} \in \mathbb{R}^{m \times 1} .
\end{aligned}
$$

Estendendo a restrição das variáveis manipuladas ao longo do horizonte de controle $\left(N_{c}\right)$, definem-se os vetores $U^{\min }$ e $U^{\max }$, conforme Equações (9) e (10) 
$U^{\min }=\left[\left(u^{\min }\right)^{T}\left(u^{\min }\right)^{T} \ldots\left(u^{\min }\right)^{T}\right]^{T} \in \mathbb{R}^{m \cdot N_{c} \times 1}$
$U^{\max }=\left[\left(u^{\max }\right)^{T}\left(u^{\max }\right)^{T} \ldots\left(u^{\max }\right)^{T}\right]^{T} \in \mathbb{R}^{m \cdot N_{c} \times 1}$.

Então, para atender à Restrição I, as variáveis manipuladas U ao longo do horizonte de controle precisam atender a um limite mínimo e máximo conforme Equação (11)

$U^{\min } \leq U \leq U^{\max }$

em que

$U=\left[\begin{array}{c}u(k) \\ u(k+1) \\ \vdots \\ u\left(k+N_{c}-1\right)\end{array}\right] \in \mathbb{R}^{m \cdot N_{c} \times 1}$

Reescrevendo (11) em duas desigualdades isolando o vetor $U$ à esquerda

$\begin{aligned}-U & \leq-U^{\min } \\ U & \leq U^{\max }\end{aligned}$

e, em seguida, reescrevendo as desigualdades em forma de matriz, tem-se as restrições expressas em relação à variável de decisão de otimização U

$\left[\begin{array}{c}-I_{m \times N_{c}} \\ I_{m \times N_{c}}\end{array}\right] U \leq\left[\begin{array}{c}-U^{\min } \\ U^{\max }\end{array}\right]$

em que $I_{m \times N_{c}}$ é a matriz identidade de dimensão $m \times N_{c}$.

Para atender à Restrição II, dado um sistema MIMO com $m$ entradas, os limites mínimos e máximos da variação do sinal de controle $\Delta u$ são dados pelos vetores $\Delta u^{\min }$ e $\Delta u^{\max }$, conforme as Equações (16) e (17)

$\Delta u^{\min }=\left[\Delta u_{1}^{\min } \Delta u_{2}^{\min } \ldots \Delta u_{m}^{\min }\right]^{T} \in \mathbb{R}^{m \times 1}$
$\Delta u^{\max }=\left[\Delta u_{1}^{\max } \Delta u_{2}^{\max } \ldots \Delta u_{m}^{\max }\right]^{T} \in \mathbb{R}^{m \times 1}$.

Estendendo a restrição das variáveis manipuladas ao longo do horizonte de controle $\left(N_{c}\right)$, definem-se os vetores $U^{\min }$ e $U^{\max }$, conforme Equações (18) e (19)

$$
\begin{aligned}
& \Delta U^{\min }=\left[\left(\Delta u^{\min }\right)^{T}\left(\Delta u^{\min }\right)^{T} \ldots\left(\Delta u^{\text {min }}\right)^{T}\right]^{T} \in \mathbb{R}^{m \cdot N_{c} \times 1} \\
& \Delta U^{\max }=\left[\left(\Delta u^{\max }\right)^{T}\left(\Delta u^{\max }\right)^{T} \ldots\left(\Delta u^{\max }\right)^{T}\right]^{T} \in \mathbb{R}^{m \cdot N_{c} \times 1} .
\end{aligned}
$$


Então, para atender à Restrição II, as variáveis manipuladas $\Delta U$ ao longo do horizonte de controle precisam atender a um limite mínimo e máximo conforme a desigualdade

$\Delta U^{\min } \leq \Delta U \leq \Delta U^{\max }$

O valor da variável manipulada $u$ no instante $k$ pode ser calculado a partir do seu valor no instante anterior $(k-1)$, acrescido de sua variação no instante $k$, conforme equação (21)

$u(k)=u(k-1)+\Delta u(k)$

Analogamente, o valor da variável manipulada $u$ no instante $(k+1)$ pode ser obtida a partir do seu valor no instante $(k-1)$, acrescido de sua variação no instante $k$ e no instante $(k+1)$

$u(k+1)=u(k-1)+\Delta u(k)+\Delta u(k+1)$.

Logo, $u(k)$ pode ser determinado a partir do seu valor no instante $(k-1)$, acrescido de sua variação ao longo do horizonte de controle $(\Delta U)$

$u(k)=u(k-1)+\left[\begin{array}{llll}I_{m \times m} & 0_{m \times m} & \ldots & 0_{m \times m}\end{array}\right] \Delta U$,

em que $\left[\begin{array}{lllll}I_{m \times m} & 0_{m \times m} & \ldots & 0_{m \times m}\end{array}\right] \in \mathbb{R}^{m \times m N_{c}}$ e $I_{m \times m}$ é a matriz identidade de dimensão $m \times m$.

De forma análoga, $u(k+1)$ pode ser determinado como

$u(k)=u(k-1)+\left[\begin{array}{llll}I_{m \times m} & I_{m \times m} & \ldots & 0_{m \times m}\end{array}\right] \Delta U$.

Logo, as ações de controle futuras ao longo do horizonte de controle podem ser relacionadas ao vetor das variações das variáveis manipuladas $(\Delta U)$, conforme apresenta a Equação (25)

$\left[\begin{array}{c}u(k) \\ u(k+1) \\ \vdots \\ u\left(k+N_{c}-1\right)\end{array}\right]=\left[\begin{array}{c}I_{m \times m} \\ I_{m \times m} \\ \vdots \\ I_{m \times m}\end{array}\right] u(k-1)+\left[\begin{array}{cccc}I_{m \times m} & 0_{m \times m} & \cdots & 0_{m \times m} \\ I_{m \times m} & I_{m \times m} & \cdots & 0_{m \times m} \\ \vdots & \vdots & \ddots & \vdots \\ I_{m \times m} & I_{m \times m} & \cdots & I_{m \times m}\end{array}\right] \Delta \mathrm{U}$ 
Renomeando as matrizes da Equação (25) como

$$
\begin{aligned}
C_{1} & =\left[\begin{array}{c}
I_{m \times m} \\
I_{m \times m} \\
\vdots \\
I_{m \times m}
\end{array}\right] \in \mathbb{R}^{m N_{c} \times m} \\
C_{2} & =\left[\begin{array}{llll}
I_{m \times m} & 0_{m \times m} & \cdots & 0_{m \times m} \\
I_{m \times m} & I_{m \times m} & \cdots & 0_{m \times m} \\
\vdots & \vdots & \ddots & 0_{m \times m} \\
I_{m \times m} & I_{m \times m} & \cdots & I_{m \times m}
\end{array}\right] \in \mathbb{R}^{m \cdot N_{c} \times m \cdot N_{c}},
\end{aligned}
$$

então esta pode ser reescrita conforme a Equação (28)

$$
U=C_{1} u(k-1)+C_{2} \Delta U
$$

Desta forma, pode-se obter $\Delta U$ através da relação inversa

$$
\Delta U=C_{2}^{-1}\left(U-C_{1} u(k-1)\right)
$$

Substituindo-se a variação da variável manipulada ao longo do horizonte de controle $(\Delta U)$ da Equação (29) na Equação (20), obtém-se a desigualdade

$$
\Delta U^{\min } \leq C_{2}^{-1} U-C_{2}^{-1} C_{1} u(k-1) \leq \Delta U^{\max } .
$$

Então, reescrevendo a Equação (30) em duas desigualdades isolando o vetor $U$ à esquerda

$$
\begin{gathered}
-C_{2}^{-1} U \leq-\Delta U^{\min }-C_{2}^{-1} C_{1} u(k-1) \\
C_{2}^{-1} U \leq \Delta U^{\max }+C_{2}^{-1} C_{1} u(k-1)
\end{gathered}
$$

e, em seguida, reescrevendo as desigualdades em forma de matriz, tem-se as restrições expressas em relação à variável de decisão de otimização U

$$
\left[\begin{array}{c}
-C_{2}^{-1} \\
C_{2}^{-1}
\end{array}\right] U \leq\left[\begin{array}{c}
-\Delta U^{\min }-C_{2}^{-1} C_{1} u(k-1) \\
\Delta U^{\max }+C_{2}^{-1} C_{1} u(k-1)
\end{array}\right]
$$

Juntando as Restrições I e II, obtem-se a desigualdade linear em função da variável manipulada $U$ 
$\left[\begin{array}{c}-I_{m \times N_{c}} \\ I_{m \times N_{c}} \\ -C_{2}^{-1} \\ C_{2}^{-1}\end{array}\right] U \leq\left[\begin{array}{c}-U^{\min } \\ U^{\max } \\ -\Delta U^{\min }-C_{2}^{-1} C_{1} u(k-1) \\ \Delta U^{\max }+C_{2}^{-1} C_{1} u(k-1)\end{array}\right]$

Para atender a Restrição III, dado um sistema MIMO com $q$ saídas, os limites mínimos e máximos da variável controlada $y$ são dados pelos vetores $y^{\mathrm{min}}$ e $y^{\text {max }}$, conforme as Equações (35) e (36)

$$
\begin{aligned}
& y^{\min }=\left[\begin{array}{llll}
y_{1}^{\min } & y_{2}^{\min } \ldots & y_{q}^{\min }
\end{array}\right]^{T} \in \mathbb{R}^{q \times 1} \\
& y^{\max }=\left[\begin{array}{llll}
y_{1}^{\max } & y_{2}^{\max } & \ldots & y_{q}^{\max }
\end{array}\right]^{T} \in \mathbb{R}^{q \times 1} .
\end{aligned}
$$

Estendendo a restrição das variáveis controladas ao longo do horizonte de predição $\left(N_{p}\right)$, definem-se os vetores $Y^{\min }$ e $Y^{\max }$, conforme Equações (37) e (38)

$$
\begin{aligned}
& Y^{\min }=\left[\left(y^{\min }\right)^{T}\left(y^{\min }\right)^{T} \ldots\left(y^{\min }\right)^{T}\right]^{T} \in \mathbb{R}^{q . N_{p} \times 1} \\
& Y^{\max }=\left[\left(y^{\max }\right)^{T}\left(y^{\max }\right)^{T} \ldots\left(y^{\max }\right)^{T}\right]^{T} \in \mathbb{R}^{q \cdot N_{p} \times 1} .
\end{aligned}
$$

Então, para atender à Restrição III, as variáveis controladas ao longo do horizonte de predição precisam atender a um limite mínimo e máximo conforme Equação (39)

$Y^{\min } \leq Y \leq Y^{\max }$

em que

$Y=\left[\begin{array}{c}y(k) \\ y(k+1) \\ \vdots \\ y\left(k+N_{p}-1\right)\end{array}\right] \in \mathbb{R}^{q \cdot N_{p} \times 1}$.

Então, reescrevendo a Equação (39) em duas desigualdades isolando os termos à esquerda

$$
\begin{aligned}
-Y+Y^{\min } & \leq 0 \\
Y-Y^{\max } & \leq 0
\end{aligned}
$$

e, em seguida, reescrevendo as desigualdades em forma de matriz, tem-se as restrições expressas conforme a desigualdade não-linear 
$\left[\begin{array}{c}-I_{q \times N_{p}} \\ I_{q \times N_{p}}\end{array}\right] Y+\left[\begin{array}{c}Y^{\min } \\ -Y^{\max }\end{array}\right] \leq 0$

em que $I_{q \times N_{p}}$ é a matriz identidade de dimensão $q \times N_{p}$.

\subsection{4.}

Sintonia do controlador

A sintonia do controle preditivo pode ser feita a partir das recomendações feitas por Seborg et al. (2011), conforme a seguir:

$N_{m} * \Delta t=t_{s}$

$30 \leq N_{m} \leq 120$

$5 \leq N_{c} \leq 20$

$\frac{N_{m}}{3} \leq N_{c} \leq \frac{N_{m}}{2}$

$N_{p}=N_{c}+N_{m}$

em que

$N_{c}$ : Horizonte de controle,

$N_{p}$ : Horizonte de predição,

$N_{m}$ : Horizonte do modelo,

$\Delta t$ : Período de amostragem,

$t_{s}:$ Tempo de assentamento.

O tempo de assentamento $\left(t_{s}\right)$ é o tempo necessário para que a saída do processo atinja e permaneça dentro de uma banda cuja largura seja igual a $\pm 5 \%$ da alteração total em y para um tempo de resposta de $95 \%$.

A faixa recomendada pelas Equações (45) e (46) garante que o modelo reflita o efeito total de uma alteração em uma variável de entrada durante o tempo necessário para atingir o estado estacionário.

Conforme o horizonte de controle $\left(N_{c}\right)$ aumenta, o controlador MPC tende a se tornar mais agressivo e o esforço computacional necessário aumenta, então valores mais baixos para $N_{c}$ são desejáveis.

O horizonte de predição $\left(N_{p}\right)$ é frequentemente selecionado para ser $N_{p}=N_{c}+N_{m}$, de modo que o efeito total do último movimento da variável 
manipulada seja levado em consideração. Diminuir o valor de $N_{p}$ tende a tornar o controlador mais agressivo.

A matriz de ponderação de saída $\left(Q_{Y}\right)$ da função custo permite que as variáveis controladas sejam ponderadas de acordo com sua importância relativa. Assim, uma matriz $Q_{y}$ diagonal de dimensão $q$ x $q$ permite que as variáveis de saída sejam ponderadas individualmente, com as variáveis mais importantes tendo os maiores pesos.

De maneira semelhante, $Q_{\Delta U}$ permite que as varáveis manipuladas sejam ponderadas de acordo com sua importância relativa. Essa matriz $m \times m$ é referida como a matriz de ponderação de entrada ou a matriz de supressão de movimento, e é geralmente escolhida para ser uma matriz diagonal. Esta fornece parâmetros de ajuste convenientes, porque aumentar o valor de $Q_{\Delta U}$ tende a tornar o controlador MPC mais conservador, reduzindo as magnitudes dos movimentos das variáveis manipuladas.

\section{4. Controle Preditivo Baseado em Modelo Não-Linear (NMPC) \\ A maioria dos processos industriais não é tão simples quanto eles são descritos em teoria; eles são não-lineares com grandes atrasos e fortes perturbações. O NMPC se torna, então, necessário para operar sob essas condições (RAO e RAWLINGS, 2002).}

\subsection{1.}

Formulação geral

Os sistemas NMPC são projetados com base em um modelo matemático não-linear que descreve o sistema. Considerando-se que o modelo não-linear tenha $m$ entradas $(u), q$ saídas $(y), n$ variáveis de estado $(x), m_{d}$ distúrbios na entrada $(v)$ e q ruídos de medição $(w)$, o modelo não-linear da planta em espaço de estado representado na forma discreta é dado pelas Equações (49) e (50)

$x(k+1)=f(x(k), u(k), v(k))$
$y(k+1)=g(x(k+1))+w(k+1)$

em que

$x \in \mathbb{R}^{n \times 1}, y \in \mathbb{R}^{q \times 1}, u \in \mathbb{R}^{m \times 1}, v \in \mathbb{R}^{m_{d} \times 1}, w \in \mathbb{R}^{q \times 1} \mathrm{e}$

$f(\cdot)$ e $g(\cdot)$ são funções não-lineares. 
Conceitualmente, a formulação do controle preditivo não-linear é semelhante ao controle preditivo linear, ou seja, encontrar um conjunto de ações de controle futuras de forma a minimizar a função objetivo (MIYOSHI, 2016) dada pela Equação (3), sujeita às restrições descritas pelas desigualdades (4), (5) e (6).

Segundo Rao e Rawlings (2002), raramente a função não-linear $f(\cdot)$ da Equação (49) está disponível na forma algébrica. Em vez disso, a função $f(\cdot)$ é vista abstratamente como a solução numérica com condição inicial $x(k)$.

\subsection{2.}

\section{Modelos internos não-lineares}

O NMPC requer a disponibilidade de um modelo dinâmico não-linear adequado para o processo. O controlador NMPC pode ser baseado em modelo não-linear fenomenológico, derivado de leis básicas de conservação e relações constitutivas, modelos não-lineares empíricos, derivados a partir de dados inferidos, e modelos não-lineares híbridos, desenvolvidos por uma combinação de abordagens de modelagem fenomenológica e empírica, permitindo que as vantagens de cada abordagem de modelagem sejam exploradas.

Segundo Henson (1998), modelos fenomenológicos têm várias vantagens em comparação com modelos empíricos. Como os modelos fenomenológicos são altamente restritos em relação a sua estrutura e parâmetros, menos dados de processo são necessários para o seu desenvolvimento. Porém, uma desvantagem potencial é que o modelo dinâmico resultante pode ser muito complexo para o design do NMPC. Em muitas aplicações, a falta de conhecimento do processo e/ou de um simulador dinâmico adequado impedem a derivação de um modelo fenomenológico. Isso exige o desenvolvimento de modelos não-lineares empíricos a partir de dados dinâmicos da planta, processo conhecido como identificação não-linear do sistema.

A compreensão detalhada do processo não é necessária para o desenvolvimento de modelos empíricos, consideração importante para processos industriais complexos, difíceis de modelar a partir de princípios fenomenológicos. Como o NMPC requer uma solução online de um problema de programação nãolinear, a sobrecarga computacional e a confiabilidade estão intimamente ligadas à complexidade do modelo não-linear. Uma vantagem dos modelos empíricos é que a forma do modelo não-linear pode ser escolhida para restringir a complexidade do modelo (HENSON, 1998). 
De acordo com Pearson e Ogunnaike (1997), uma dificuldade fundamental associada à abordagem de modelagem empírica é a seleção de um modelo adequado. Os tipos de modelos não-lineares mais utilizados para NMPC são

○ Volterra,

- Hammerstein,

- Wiener,

- Redes Neurais.

Para sistemas de colunas de destilação, são comumente utilizados os modelos de Hammerstein e Redes Neurais, porque combinam simplicidade de modelagem com boa aproximação do comportamento não-linear real do processo.

\subsubsection{1. Aplicações do modelo de Hammerstein para colunas de destilação}

$\mathrm{Na}$ literatura, são encontradas várias aplicações nas quais o modelo de Hammerstein é utilizado para representar o sistema físico de uma coluna de destilação, apresentadas a seguir.

Rangaretnam e Riggs (1998) aplicaram o modelo de Hammerstein com bloco estacionário não-linear modelado por Redes Neurais a uma coluna de destilação de alta pureza, para um sistema SISO, com o propósito de aplicar um controlador NMPC e compará-lo ao controlador DMC e controlador PI. O NMPC mostrou melhor desempenho em comparação aos outros controladores.

Santoso et al. (2009) fizeram a análise da operabilidade dinâmica de uma coluna de destilação reativa com aplicação de um controlador ótimo, para um sistema MIMO. Para tal o modelo usado para a identificação do sistema e implementação do NMPC foi o Hammerstein.

Marusak (2010) desenvolveu um novo método de predição com NMPC por modelo de Hammerstein com bloco estacionário não-linear modelado por Fuzzy e testa a aplicação a uma coluna de destilação, em um sistema SISO.

Biagiola e Figueroa (2011) estudaram a identificação de uma coluna de destilação por modelos MIMO de Wiener e Hammerstein, em que ambos os modelos apresentaram identificação robusta do sistema.

Yusoff et al. (2011) compararam a aplicação de 3 estratégias de NMPC a um sistema SISO de destilação a vapor, com os modelos de Hammerstein, Wiener 
e Hammerstein-Winer. O Modelo que mostrou melhor ajuste e menor perda de função foi o Hammerstein-Wiener.

Kanthasamy et al. (2014) aplicou um NMPC com modelo de Hammerstein com bloco estacionário não-linear modelado por wavenet e bloco linear dinâmico por erro de saída a uma coluna de destilação. A validação do modelo mostrou que este foi capaz de capturar as não-linearidades do sistema.

Aggoune e Chetouani (2017) compararam os modelos de Hammerstein e NARMAX para a modelagem de uma coluna de destilação MISO. Os resultados mostraram que ambos os modelos produziram predições aceitáveis, mas que 0 modelo NARMAX superou o modelo de Hammerstein.

A Tabela 3 resume os trabalhos encontrados na literatura em que o modelo de Hammerstein foi utilizado para representar uma coluna de destilação.

Tabela 3: Resumo dos trabalhos encontrados na literatura em que o modelo de Hammerstein foi utilizado para representar uma coluna de destilação.

\begin{tabular}{llllll}
\hline Autor & Ano & Sistema & Tipo de coluna & Aplicação & Comparação \\
\hline Rangaretnam e Riggs & 1998 & SISO & alta pureza & NMPC & Controladores NMPC, DMC e PI \\
Santoso et al. & 2009 & MIMO & destilação reativa & NMPC & - \\
Marusak & 2010 & SISO & simples & Modelagem & - \\
Biagiola e Figueroa & 2011 & MIMO & simples & Modelagem & Modelos Hammerstein and Wiener \\
Yusoff et al. & 2011 & SISO & a vapor & NMPC & Hammerstein-Winer \\
Kanthasamy et al. & 2014 & MISO & simples & NMPC & - Wiener e \\
Aggoune e Chetouani & 2017 & MISO & simples & Modelagem & Modelos Hammerstein e NARMAX \\
\hline
\end{tabular}

\subsubsection{2.}

\section{Aplicações do modelo de Redes Neurais para colunas de destilação}

Aplicações nas quais o modelo de Redes Neurais é utilizado para representar o sistema físico de uma coluna de destilação são apresentadas a seguir.

MacMurray e Himmelblau (1995) modelaram um sistema MIMO de coluna de destilação recheada por modelo de Redes Neurais e concluíram que o modelo é tão bom ou melhor que um modelo simplificado quando usado para controle preditivo baseado em modelo.

Jazayeri-Rad (2004) usou uma combinação de múltiplas redes neurais para modelar sistemas MIMO não-lineares para aplicação de NMPC. O esquema proposto foi aplicado e avaliado para dois exemplos de problemas, incluindo o NMPC de uma coluna de destilação de múltiplos componentes, demonstrando excelente performance de controle. 
Ławryńczuk e Tatjewski (2006) aplicaram NMPC com modelo de redes neurais MIMO a uma coluna de destilação de alta pureza. Em comparação com a técnica geral não-linear do MPC, a estrutura apresentada é muito mais confiável e demanda menos esforço computacional.

Chetouani (2007) aplicou o modelo de redes neurais MIMO para identificar uma coluna de destilação, em que o modelo prediz com sucesso a composição dos produtos.

Ławryńczuk (2009) aplicou um controlador NMPC com modelo de redes neurais MIMO a uma coluna de destilação. O algoritmo usa a linearização do modelo neural em torno do ponto operacional. $O$ algoritmo é computacionalmente eficiente porque a política de controle é calculada explicitamente sem qualquer otimização. Para a coluna de destilação considerada, a precisão do controle é comparável para o NMPC com linearização do modelo de redes neurais como também àquela obtida no NMPC com otimização.

Sharma e Singh (2012) compararam a aplicação de 3 estratégias de controle em uma coluna de destilação reativa TAME, sendo essas controle SISO por PID, controle MPC e controle NMPC com modelo de redes neurais. As estratégias foram comparadas e concluiu-se que o NMPC e o MPC ofereceram melhor desempenho no controle.

Sharma e Singh (2013) compararam a aplicação de 3 estratégias de controle em uma coluna de destilação reativa TAME, sendo essas controle SISO por PID, controle NMPC com modelo de redes neurais e NMPC com modelo de support vector machine. As estratégias foram comparadas e concluiu-se que as estratégias NMPC ofereceram melhor desempenho no controle.

Giwa e Giwa (2013) aplicaram um modelo de redes neurais a um processo de destilação reativo SISO, em que a validação do modelo confirmou sua eficiência de predição.

Ramli et al. (2016) aplicaram um modelo de rede neural baseado em equações representado por uma equação multivariável MIMO a uma coluna de destilação. Os resultados mostram que o modelo baseado em redes neurais na abordagem de modelos direto e inverso apresenta melhor desempenho do que 0 método PID convencional em ambos os casos.

Kataria e Singh (2017) propuseram um soft sensor baseado em Rede Neural Recorrente (RNN) SISO para estimar a composição do produto de fundo de uma Coluna de Destilação Reativa. Os resultados mostraram que o soft sensor estimou a composição no fundo da coluna com uma precisão que pode ser usada para fins de controle. 
Hieu et al. (2017) aplicaram NMPC com modelo de redes neurais com princípio de horizonte móvel para controle de um sistema de coluna de destilação, num sistema MIMO. Os resultados da simulação indicaram que o NMPC é capaz de rastrear as trajetórias de referência e rejeitar os distúrbios.

Heras-Cervantes et al. (2018) apresentam a validação de um modelo SISO baseado em redes neurais para estimação online da composição do componente leve de uma coluna de destilação descontínua para uma mistura binária. Os resultados obtidos foram comparados com os de um modelo não-linear fenomenológico, em que o modelo por redes neurais maior simplicidade de programação e implementação.

A Tabela 4 resume os trabalhos encontrados na literatura em que o modelo de Redes Neurais foi utilizado para representar uma coluna de destilação.

Tabela 4: Resumo dos trabalhos encontrados na literatura em que o modelo de Redes Neurais foi utilizado para representar uma coluna de destilação.

\begin{tabular}{llllll}
\hline Autor & Ano & Sistema & Tipo de coluna & Aplicação & Comparação \\
\hline MacMurray e Himmelblau & 1995 & MIMO & recheada & NMPC & MPC \\
Jazayeri-Rad & 2004 & MIMO & multi-componentes & NMPC & - \\
Ławryńczuk e Tatjewski & 2006 & MIMO & alta pureza & NMPC & NMPC e GPC \\
Chetouani & 2007 & MIMO & simples & modelagem & - \\
Ławryńczuk & 2009 & MIMO & simples & NMPC & NMPC não linear e linearizado \\
Sharma e Singh & 2012 & SISO & reativa TAME & NMPC & NMPC, MPC e PID \\
Sharma e Singh & 2013 & SISO & reativa TAME & NMPC & RNA e SVM \\
Giwa e Giwa & 2013 & SISO & reativa & Modelagem & - \\
Ramli et al. & 2016 & MIMO & simples & NMPC & NMPC e PID \\
Kataria e Singh & 2017 & SISO & reativa & soft-sensor & - \\
Hieu et al. & 2017 & MIMO & simples & NMPC & - \\
Heras-Cervantes et al. & 2018 & SISO & descontínua & modelagem & RNA e fenomenológico
\end{tabular}

\subsection{3.}

\section{Controle Preditivo baseado em Modelo de Hammerstein Modificado}

Esta seção apresenta primeiramente 0 modelo de Hammerstein Modificado. A partir deste, é construído o modelo interno do controlador para um horizonte de predição, cálculos para a função objetivo e cálculos para o Estimador de Horizonte Móvel. 


\subsubsection{1.}

\section{Modelo de Hammerstein Modificado}

Modelos orientados a bloco do tipo Hammerstein são modelos de fácil descrição matemática pois possuem um bloco linear dinâmico e um não-linear estacionário independentes. O modelo de Hammerstein modificado proposto por Miyoshi (2016), apresentado nesta seção, propõe uma estrutura com uma mudança de variável de forma a obter uma solução analítica do problema de predição não-linear futura com maior rapidez computacional dos cálculos matriciais.

Dado um sistema que possua $m$ entradas $(u), q$ saídas $(y), n$ variáveis de estado $(x)$ e $m_{d}$ distúrbios na entrada $(v)$, a variável manipulada é descrita por uma função polinomial não-linear $\phi$, em função da entrada $u$, com $p$ coeficientes. Esta função pode ser decomposta em $p$ funções $\left\{\phi_{1}(u), \phi_{2}(u), \ldots, \phi_{p}(u)\right\}$, em que $u=\left[u_{1}, u_{2}, \ldots, u_{m}\right]^{T} \in \mathbb{R}^{m \times 1}$. Então, a função polinomial $\phi(u, k)$, em função da entrada $u$ e do instante $k$, pode ser descrita conforme a Equação (51)

$\phi(u, k)=\left[\begin{array}{c}\phi_{1}\left(u_{1}(k)\right) \\ \phi_{2}\left(u_{1}(k)\right) \\ \vdots \\ \phi_{p}\left(u_{1}(k)\right) \\ \vdots \\ \phi_{1}\left(u_{m}(k)\right) \\ \phi_{2}\left(u_{m}(k)\right) \\ \vdots \\ \phi_{p}\left(u_{m}(k)\right)\end{array}\right] \in \mathbb{R}^{m \cdot p \times 1}$

Estruturas de controle preditivo se beneficiam da rapidez computacional dos cálculos matriciais dessa estrutura analítica. A estrutura é linear em relação a $\phi(u, k)$, mas pode apresentar não-linearidades em relação às variáveis manipuladas $(u)$, dependendo da função de base $\phi(u)$. A estrutura do modelo interno do controlador em espaço de estado, no domínio discreto de tempo, considerando o modelo de Hammerstein modificado, pode ser descrita conforme as Equações (52) e (53)

$$
\begin{aligned}
& x(k+1)=A x(k)+B_{\text {mod }} \phi(u, k)+B_{v} v(k) \\
& y(k+1)=C x(k+1),
\end{aligned}
$$


em que

$x \in \mathbb{R}^{n \times 1}, y \in \mathbb{R}^{q \times 1}, u \in \mathbb{R}^{m \times 1}, v \in \mathbb{R}^{m_{d} \times 1}, \phi \in \mathbb{R}^{m . p \times 1} \mathrm{e}$

$A \in \mathbb{R}^{n \times n}, B_{\text {mod }} \in \mathbb{R}^{n \times m . p}, B_{v} \in \mathbb{R}^{n \times m_{d}}, C \in \mathbb{R}^{q \times n}$ são as matrizes no espaço de estado com a variável de entrada modificada e $B_{\text {mod }}$ é descrita conforme Equação (54)

$B_{\text {mod }}=\left[\begin{array}{ccccccc}b_{1,1} & b_{1,2} & \cdots & b_{1,(m-1) p+1} & b_{1,(m-1) p+2} & \cdots & b_{1,(m-1) p+p} \\ \vdots & & & & & & \\ b_{n, 1} & b_{n} & \cdots & b_{n,(m-1) p+1} & b_{n,(m-1) p+2} & \cdots & b_{n,(m-1) p+p}\end{array}\right]$.

Logo, a mudança de variável pode representar a não-linearidade estática em uma forma linear conforme o modelo de Hammestein modificado da Figura 6.

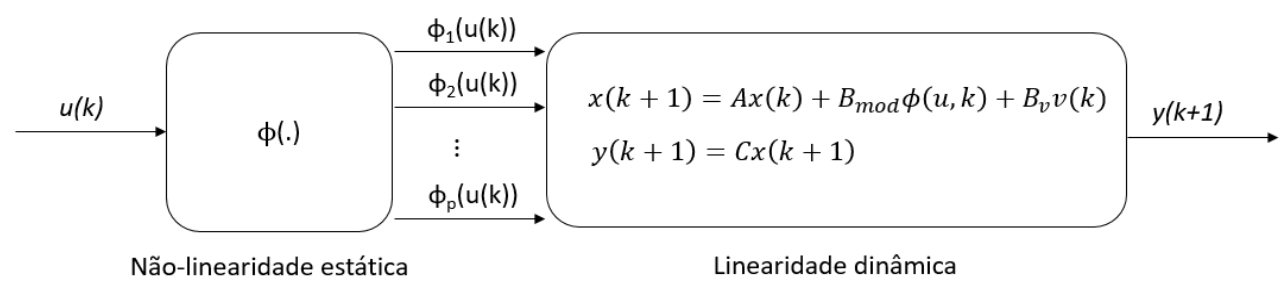

Figura 6: Modelo de Hammerstein modificado.

\subsubsection{2.}

\section{Modelo interno do controlador H-NMPC}

O modelo interno do controlador para o horizonte de predição $N_{p}$ utiliza estrutura similar à utilizada para o controle linear, em que o valor predito das variáveis de saída $(y)$ no horizonte de predição até $N_{p}$ passos à frente é dado por

$Y=\left[\begin{array}{c}y(k) \\ y(k+1) \\ \vdots \\ y\left(k+N_{p}-1\right)\end{array}\right] \in \mathbb{R}^{q . N_{p} \times 1}$

em que a relação obtida para a predição de $y(k+i)$ até um horizonte de predição $N_{p}$, tal que $0 \leq \mathrm{i} \leq N_{p}-1$

$$
\begin{aligned}
& y(k+i)=C A^{i} x(k)+C A^{i-1} B_{\text {mod }} \phi(k)+C A^{i-2} B_{\text {mod }} \phi(k+1)+C A^{i-i} B_{\text {mod }} \phi(k+ \\
& i-1)+\cdots+C A^{i-1} B_{v} v(k)+C A^{i-2} B_{v} v(k+1)+\cdots+C A^{i-i} B_{v} v(k+i-1) .
\end{aligned}
$$


Com o intuito de se obter uma expressão analítica matricial, para as saídas (y) preditas em um horizonte de predição $N_{p}$, definem-se as ações de controle modificadas para um horizonte de controle $N_{c}$

$\Phi_{\text {mod }}=\left[\begin{array}{c}\phi_{1}\left(u_{1}(k)\right) \\ \phi_{2}\left(u_{1}(k)\right) \\ \vdots \\ \phi_{p}\left(u_{1}(k)\right) \\ \vdots \\ \phi_{1}\left(u_{m}(k)\right) \\ \phi_{2}\left(u_{m}(k)\right) \\ \vdots \\ \phi_{p}\left(u_{m}(k)\right) \\ \vdots \\ \phi_{1}\left(u_{1}\left(k+N_{c}-1\right)\right) \\ \phi_{2}\left(u_{1}\left(k+N_{c}-1\right)\right) \\ \vdots \\ \phi_{p}\left(u_{1}\left(k+N_{c}-1\right)\right) \\ \vdots \\ \phi_{1}\left(u_{m}\left(k+N_{c}-1\right)\right) \\ \phi_{2}\left(u_{m}\left(k+N_{c}-1\right)\right) \\ \vdots \\ \phi_{p}\left(u_{m}\left(k+N_{c}-1\right)\right)\end{array}\right] \in \mathbb{R}^{m \cdot p \cdot N_{c} \times 1}$.

O distúrbio medido $v(k)$, constante em todo o horizonte de predição $N_{p}$, é dado por

$v(k)=v(k+1)=v\left(k+N_{p}-1\right)$.

Substituindo-se as Equações (57)-(58) nas expressões de predição das saídas descritas pelos conjuntos de Equações (55)-(56), obtém-se uma forma matricial dessas equações

$Y=F x(k)+\Omega_{\text {mod }} \Phi_{\text {mod }}+\Psi v(k)$

em que

$F=\left[\begin{array}{c}C A \\ C A^{2} \\ C A^{3} \\ \vdots \\ C A^{N_{p}}\end{array}\right] \in \mathbb{R}^{q \cdot N_{p} \times \mathrm{n}}$

$\Omega_{\text {mod }}=\left[\begin{array}{ccccc}C B_{\text {mod }} & 0 & 0 & \cdots & 0 \\ C A B_{\text {mod }} & C B_{\text {mod }} & 0 & \cdots & 0 \\ C A^{2} B_{\text {mod }} & C A B_{\text {mod }} & C B_{\text {mod }} & \cdots & 0 \\ \vdots & \vdots & \vdots & \ddots & \vdots \\ C A^{N_{p}-1} B_{\text {mod }} & C A^{N_{p}-2} B_{\text {mod }} & C A^{N_{p}-3} B_{\text {mod }} & \cdots & C A^{N_{p}-N_{-} c} B_{\text {mod }}\end{array}\right]$ 
$\Omega_{\text {mod }} \in \mathbb{R}^{q \cdot N_{p} \times \text { m.p. } \mathrm{N}_{\mathrm{c}}}$

$\Psi=\left[\begin{array}{c}C B_{v} \\ C A B_{v}+C B_{v} \\ C A^{2} B_{v}+C A B_{v}+C B_{v} \\ \vdots \\ C A^{N_{p}-1} B_{v}+C A^{N_{p}-2} B_{v}+C A^{N_{p}-3} B_{v}+\cdots+C B_{v}\end{array}\right] \in \mathbb{R}^{q . N_{p} \times \mathrm{m}_{\mathrm{d}}}$

\subsubsection{3.}

Função objetivo para modelo de Hammerstein

A função objetivo proposta por Wang (2009), e discutida na Seção 2.3.3., é dada por

$J=0.5\left(Y^{s p}-Y\right)^{T} Q_{Y}\left(Y^{s p}-Y\right)+0.5 \Delta U^{T} Q_{\Delta U} \Delta U$

em que

$\Delta U=C_{2}^{-1}\left(U-C_{1} u(k-1)\right)$

$C_{1}=\left[\begin{array}{c}I_{m \times m} \\ I_{m \times m} \\ \vdots \\ I_{m \times m}\end{array}\right] \in \mathbb{R}^{m N_{c} \times m}$

$C_{2}=\left[\begin{array}{llll}I_{m \times m} & 0_{m \times m} & \cdots & 0_{m \times m} \\ I_{m \times m} & I_{m \times m} & \cdots & 0_{m \times m} \\ \vdots & \vdots & \ddots & 0_{m \times m} \\ I_{m \times m} & I_{m \times m} & \cdots & I_{m \times m}\end{array}\right] \in \mathbb{R}^{m \cdot N_{c} \times m \cdot N_{c}}$

Substituindo a Equação (64) na Equação (63), temos

$J=\left(Y^{s p}-Y\right)^{T} Q_{Y}\left(Y^{s p}-Y\right)+\left[C_{2}^{-1}\left(U-C_{1} u(k-1)\right)\right]^{T} Q_{\Delta U}\left[C_{2}^{-1}\left(U-C_{1} u(k-1)\right)\right]$.

A função objetivo está sujeita às restrições descritas pelas desigualdades 


$$
\begin{aligned}
& {\left[\begin{array}{c}
-I_{m \times N_{c}} \\
I_{m \times N_{c}} \\
-C_{2}^{-1} \\
C_{2}^{-1}
\end{array}\right] U \leq\left[\begin{array}{c}
-U^{\min } \\
U^{\max } \\
-\Delta U^{\min }-C_{2}^{-1} C_{1} u(k-1) \\
-\Delta U^{\min }-C_{2}^{-1} C_{1} u(k-1)
\end{array}\right]} \\
& {\left[\begin{array}{c}
-I_{q \times N_{p}} \\
I_{q \times N_{p}}
\end{array}\right] Y+\left[\begin{array}{c}
Y^{\min } \\
-Y^{\max }
\end{array}\right] \leq 0 .}
\end{aligned}
$$

Para o modelo de Hammerstein modificado, a saída predita é dada pela Equação (70) proposta por Miyoshi (2016), conforme discutido na Seção 2.4.3.

$Y=F x(k)+\Omega_{\text {mod }} \Phi_{\text {mod }}+\Psi v(k)$

Substituindo a Equação (70) na Equação (67), temos

$2 J=\left(Y^{s p}-F x(k)+\Omega_{\text {mod }} \Phi_{\text {mod }}+\Psi v(k)\right)^{T} Q_{Y}\left(Y^{s p}-F x(k)+\Omega_{\text {mod }} \Phi_{\text {mod }}+\right.$ $\Psi v(k))+\left(U-C_{1} u(k-1)\right)^{T}\left(C_{2}^{-1}\right)^{T} Q_{\Delta U} C_{2}^{-1}\left(U-C_{1} u(k-1)\right)$.

Agrupando os termos referentes à variável manipulada $(U)$ e à variável manipulada modificada $\Phi_{\text {mod }}$, temos

$2 J=\left(Y^{s p}-F x(k)+\Psi v(k)\right)^{T} Q_{Y}\left(Y^{s p}-F x(k)+\Psi v(k)\right)-2 \Phi_{\text {mod }}^{T} \Omega_{\text {mod }}^{T} Q_{y}\left(Y^{s p}-\right.$

$F x(k)+\Psi v(k))+\Phi_{\text {mod }}^{T}\left(\Omega_{\text {mod }}^{T} Q_{y} \Omega_{\text {mod }}\right) \Phi_{\text {mod }}+\left(C_{1} u(k-\right.$

1)) $)^{T}\left(C_{2}^{-1}\right)^{T} Q_{\Delta U} C_{2}^{-1}\left(C_{1} u(k-1)\right)-2 U^{T}\left(C_{2}^{-1}\right)^{T} Q_{\Delta U} C_{2}^{-1}\left(C_{1} u(k-1)\right)+$

$U^{T}\left(C_{2}^{-1}\right)^{T} Q_{\Delta U} C_{2}^{-1} U$.

Elimina-se os termos constantes da equação, uma vez que estes não afetam o argumento que minimiza o funcional $J$

$2 J=-2 \Phi_{\text {mod }}^{T} \Omega_{\text {mod }}^{T} Q_{y}\left(Y^{s p}-F x(k)+\Psi v(k)\right)+\Phi_{\text {mod }}^{T}\left(\Omega_{\text {mod }}^{T} Q_{y} \Omega_{\text {mod }}\right) \Phi_{\text {mod }}-$
$2 U^{T}\left(C_{2}^{-1}\right)^{T} Q_{\Delta U} C_{2}^{-1}\left(C_{1} u(k-1)\right)+U^{T}\left(C_{2}^{-1}\right)^{T} Q_{\Delta U} C_{2}^{-1} U$.

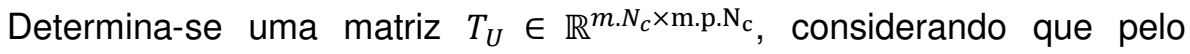
menos uma função de base $\left(\phi_{i}\right)$ seja inversível de forma a determinar uma matriz $T_{U} \in \mathbb{R}^{m \cdot N_{c} \times m . p . N_{c}}$, tal que 
$U=T_{U} \Phi_{m o d}$

ou seja,

$\left[\begin{array}{c}u(k) \\ u(k+1) \\ \vdots \\ u\left(k+N_{c}-1\right)\end{array}\right]=\left[\begin{array}{ccccccc}1 & 0 & 0 & 0 & \cdots & 0 & 0 \\ 0 & 0 & 1 & 0 & \cdots & 0 & 0 \\ \vdots & \vdots & \vdots & \vdots & \ddots & \vdots & \vdots \\ 0 & 0 & 0 & 0 & \cdots & 1 & 0\end{array}\right]\left[\begin{array}{c}u_{1}(k) \\ u_{1}^{2}(k) \\ u_{1}(k+1) \\ u_{1}^{2}(k+1) \\ \vdots \\ u_{1}\left(k+N_{c}-1\right) \\ u_{1}^{2}\left(k+N_{c}-1\right)\end{array}\right]$.

Substituindo então a Equação (74) na Equação (75), temos

$2 J=-2 \Phi_{\text {mod }}^{T}\left[\Omega_{\text {mod }}^{T} Q_{y}\left(Y^{s p}-F x(k)+\Psi v(k)\right)+T_{U}^{T}\left(C_{2}^{-1}\right)^{T} Q_{\Delta U} C_{2}^{-1}\left(C_{1} u(k-1)\right)\right]+$ $\Phi_{\text {mod }}^{T}\left[\Omega_{\text {mod }}^{T} Q_{y} \Omega_{\text {mod }}+T_{U}^{T}\left(C_{2}^{-1}\right)^{T} Q_{\Delta U} C_{2}^{-1} T_{U}\right] \Phi_{\text {mod }}$.

A função objetivo pode ser simplificada conforme a Equação (77)

$J=0.5\left(-2 \Phi_{\text {mod }}^{T} c_{\text {mod }}+\Phi_{\text {mod }}^{T} H_{\text {mod }} \Phi_{\text {mod }}\right)$

em que

$c_{\text {mod }}=\Omega_{\text {mod }}^{T} Q_{y}\left(Y^{s p}-F x(k)+\Psi v(k)\right)+T_{U}^{T}\left(C_{2}^{-1}\right)^{T} Q_{\Delta U} C_{2}^{-1}\left(C_{1} u(k-1)\right)$

$H_{\text {mod }}=\Omega_{\text {mod }}^{T} Q_{y} \Omega_{\text {mod }}+T_{U}^{T}\left(C_{2}^{-1}\right)^{T} Q_{\Delta U} C_{2}^{-1} T_{U}$.

Essa simplificação da função objetivo é feita de forma a computar previamente os termos $c_{\text {mod }}$ e $H_{\text {mod }}$ em vez de serem calculados a cada iteração, aumentando a velocidade computacional da convergência do algoritmo de otimização.

Para fazer a solução analítica da otimização, calcula-se o gradiente da função objetivo e a Jacobiana das restrições, de forma a obter a função Lagrangiana. Assim, o vetor gradiente da função objetivo é obtido pela regra da cadeia

$\nabla_{U}(J)=-\left(c_{\text {mod }}-H_{\text {mod }} \Phi_{\text {mod }}\right) \nabla_{U}\left(\Phi_{\text {mod }}\right)$. 
$H(J)=\nabla_{U}^{T}\left(\Phi_{\text {mod }}\right) H_{\text {mod }}^{T} \nabla_{U}\left(\Phi_{\text {mod }}\right)-c_{\text {mod }}^{T} H\left(\Phi_{\text {mod }}\right)$,

em que

$\nabla_{U} \Phi_{m o d}=$
$\left[\begin{array}{ccclc}\nabla_{u(k)} \phi(k) & 0_{m . p \times m} & 0_{m . p \times m} & \cdots & 0_{m . p \times m} \\ 0_{m . p \times m} & \nabla_{u(k+1)} \phi(k+1) & 0_{m . p \times m} & \cdots & 0_{m . p \times m} \\ 0_{m . p \times m} & 0_{m . p \times m} & \nabla_{u(k+2)} \phi(k+2) & \cdots & 0_{m . p \times m} \\ \vdots & \vdots & \vdots & \ddots & \vdots \\ 0_{m . p \times m} & 0_{m . p \times m} & 0_{m . p \times m} & \cdots & \nabla_{u\left(k+N_{c}-1\right)} \phi\left(k+N_{c}-1\right)\end{array}\right]$.

A restrição não-linear é dada substituindo a Equação (70) na Equação (71) e reescrevendo a desigualdade expressa em relação à variável de controle modificada $\Phi_{\text {mod }}$, conforme equação

$\left[\begin{array}{c}-\Omega_{\text {mod }} \\ \Omega_{\text {mod }}\end{array}\right] \Phi_{\text {mod }}-\left[\begin{array}{c}-Y^{\min }+\Psi v(k)+F x(k) \\ Y^{\max }-\Psi v(k)-F x(k)\end{array}\right] \leq 0$

a qual pode ser reescrita como

$c_{3}=M_{3} \Phi_{\text {mod }}-\gamma_{3} \leq 0$

Portanto, a matriz jacobiana do vetor de restrições não-lineares é dada por

$\nabla_{U}\left(c_{3}\right)=M_{3} \nabla_{U}\left(\Phi_{\text {mod }}\right)$

em que

$\nabla_{U}\left(c_{3}\right) \in \mathbb{R}^{2 q N_{p} \times m . N_{c}}$

$M_{3} \in \mathbb{R}^{2 q N_{p} \times m \cdot N_{c}}$.

A matriz hessiana da i-ésima restrição de $2 q N_{p}$ restrições não-lineares é dada por

$\mathrm{H}_{3, i}=H\left(M_{3}(i,:) \Phi_{\text {mod }} \in \mathbb{R}^{m N_{c} \times m \cdot N_{c}}\right.$. 


\subsubsection{4.}

\section{Estimador de Horizonte Móvel para modelo de Hammerstein}

O Estimador de Horizonte Móvel (MHE - do inglês, Moving Horizon Estimation) é uma estratégia baseada em otimização para monitoramento de processos e estimação de estado. O MHE pode ser visto como uma extensão para o filtro de Kalman para processos não-lineares com restrições. O MHE, portanto, inclui tanto o filtro de Kalman quanto o filtro de Kalman estendido. Além disso, o MHE permite incluir restrições no problema de estimação, melhorando significativamente a qualidade da estimação de estado para certos problemas, de forma a incorporar conhecimento prévio na forma de desigualdades de restrições. As desigualdades de restrições fornecem uma ferramenta flexível para complementar o conhecimento do processo e como estratégia para simplificação de modelos. A capacidade de incluir restrições e dinâmica não-linear é o que distingue o MHE de outras estratégias de estimativa (RAO e RAWLINGS, 2002).

Miyoshi (2016) propõe resolver para a janela de horizonte $N_{j a n}$ o seguinte problema matemático para o modelo de Hammerstein modificado

$$
\min _{\gamma_{\mathrm{MHE}}} J_{M H E}=-2 \gamma_{M H E}^{T} f_{M H E}+\gamma_{M H E}^{T} H_{M H E} \gamma_{M H E}
$$

sujeito às restrições

$M_{M H E} \gamma_{\mathrm{MHE}} \leq \gamma_{\mathrm{MHE}}$

$f_{M H E}=\left(F_{2}^{T} R_{M H E}^{-1}\left(Y_{m}-\Omega_{M H E} \Phi_{\text {mod }}(u)\right)+F_{4}^{T} \Pi_{k-N_{j a n}}^{-1} x_{k-N_{j a n}}\right.$

$H_{M H E}=F_{2}^{T} R_{M H E}^{-1} F_{2}+F_{3}^{T} Q_{M H E}^{-1} F_{3}+F_{4}^{T} \Pi_{k-N_{j a n}}^{-1} F_{4}$.

Q e R são os parâmetros de ajuste para reconciliar o modelo com as medições do processo. As matrizes fornecem meios pelos quais os erros são distribuídos entre o modelo e os sensores do processo. A matriz $Q$ modela a incerteza no modelo, enquanto a matriz $R$ fornece uma medida de confiança nos sensores do processo. Assim, se a matriz $Q$ é "grande" em relação a $R$, então estamos menos confiantes no modelo do que nos sensores de processo e viceversa. A matriz $\Pi$ fornece uma medida de confiança do estado inicial (RAO e RAWLINGS, 2002). 
$y_{m}$ é o valor medido da variável de saída. $v(k)$ é o ruído do modelo do processo, assumindo-se uma distribuição normal multivariada de média zero e covariância $Q(k)$

$v(k) \sim N(0, Q(k))$

e $w_{M H E}(k)$ é o ruído da observação, assumindo-se um ruído branco gaussiano de média zero e covariância $R(k)$

$w_{M H E}(k) \sim N(0, R(k))$.

em que

$$
\begin{aligned}
& \mathrm{Y}_{m}=\left[\begin{array}{llll}
y_{m}^{T}\left(k-N_{j a n}+1\right) & y_{m}^{T}\left(k-N_{j a n}+2\right) & \ldots & y_{m}^{T}(k)
\end{array}\right]^{T} \in \mathbb{R}^{q . N_{j a n} \times 1} \\
& \mathrm{~W}=\left[\begin{array}{llll}
w_{M H E}^{T}\left(k-N_{j a n}+1\right) & w_{M H E}^{T}\left(k-N_{j a n}+2\right) & \ldots & w_{M H E}^{T}(k)
\end{array}\right]^{T} \in \mathbb{R}^{q . N_{j a n} \times 1} \\
& \gamma=\left[\begin{array}{llll}
v_{M H E}^{T}\left(k-N_{j a n}+1\right) & v_{M H E}^{T}\left(k-N_{j a n}+2\right) & \ldots & v_{M H E}^{T}(k)
\end{array}\right]^{T} \in \mathbb{R}^{n . N_{j a n} \times 1} .
\end{aligned}
$$

A estrutura proposta do H-NMPC é observada na Figura 7, em que o MHE recebe o valor medido da variável de saída $y_{m}$ mais um distúrbio $d_{m}$ e estima 0 estado $\hat{x}$ para entrada no H-NMPC, o qual calcula o melhor sinal de entrada $u$ para a camada de controle regulatório da planta.

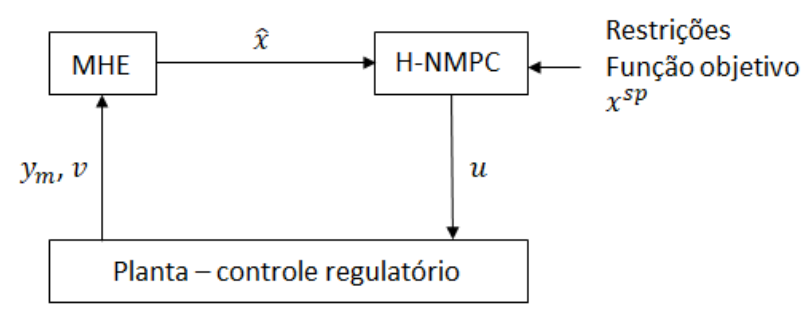

Figura 7: Estrutura do H-NMPC.

\subsection{4.}

\section{Controle Preditivo baseado em Modelo de Redes Neurais}

Esta seção apresenta o modelo de Redes Neurais e o modelo interno do controlador para um horizonte de predição. 


\subsubsection{1.}

\section{Modelo de Redes Neurais}

A característica mais atraente das Redes Neurais, (NN - do inglês Neural Networks), e também a fonte de sua reputação como ferramentas poderosas para resolver diversos problemas, é sua alta capacidade de mapear sistemas nãolineares, capacitando-os a aprender os comportamentos inerentes a partir de dados adquiridos de tais sistemas.

A ponderação realizada pelas junções sinápticas da rede é implementada no neurônio artificial como um conjunto de pesos sinápticos. Analogamente, a relevância de cada uma das entradas dos neurônios é calculada multiplicando-as pelo seu peso sináptico correspondente, ponderando assim toda a informação externa que chega ao neurônio (SILVA et al., 2017). Portanto, a saída de cada neurônio é a soma ponderada de suas entradas, conforme Figura 8.

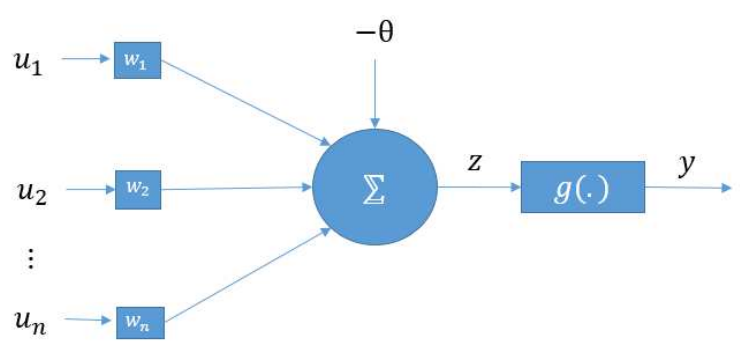

Figura 8: O neurônio artificial.

As Equações (98) e (99) sintetizam o algoritmo produzido pelos neurônios artificiais para $n$ entradas

$$
\begin{aligned}
& \mathrm{z}=\sum_{i=1}^{n} u_{i} w_{i}-\theta \\
& y=g(z)
\end{aligned}
$$

em que

- $u$ : sinais de entrada da rede

- $\quad w$ : são os valores utilizados para ponderar cada uma das variáveis de entrada, o que permite quantificar sua relevância em relação à funcionalidade do neurônio.

- $\quad \mathbb{E}$ : agregador linear, reúne todos os sinais de entrada ponderados pelos pesos sinápticos para produzir uma tensão de ativação. 
- $\theta$ : limite de ativação ou bias, é uma variável usada para especificar o limite adequado que o resultado produzido pelo agregador linear deve ter para gerar um valor de disparo em direção à saída do neurônio.

- $\quad z$ : potencial de ativação, é o resultado produzido pela diferença entre o agregador linear e o limite de ativação. Se este valor for positivo, isto é, se $z \geq \theta$, então o neurônio produz um potencial excitatório; caso contrário, será inibitório.

- $g$ : função de ativação, cujo objetivo é limitar a saída do neurônio dentro de uma faixa razoável de valores, assumidos por sua própria imagem funcional.

- $y$ : sinal de saída, consiste no valor final produzido pelo neurônio dado um conjunto particular de sinais de entrada, e também pode ser usado como entrada para outros neurônios interconectados sequencialmente.

\subsubsection{2. \\ Modelo interno do controlador}

Singh et al. (2014) propõe um Controle Preditivo baseado em Modelo de Redes Neurais (NN-NMPC) para controle de uma coluna de destilação. A estrutura do NN-NMPC é usada para prever saídas futuras passos a frente num horizonte de predicao $N_{p}$.

O modelo proposto tem como entradas da rede neural, com apenas uma camada escondida com $n$ neurônios, a variável de estado do sistema $(k)$ e duas variáveis de controle $\left(u_{1}\right.$ e $\left.u_{2}\right)$ no instante atual $(k)$ e no instante passado $(k-1)$ conforme a Equação (100)

$x(k+1)=f\left(x(k), x(k-1), u_{1}(k), u_{1}(k-1), u_{2}(k), u_{2}(k-1)\right)$. 


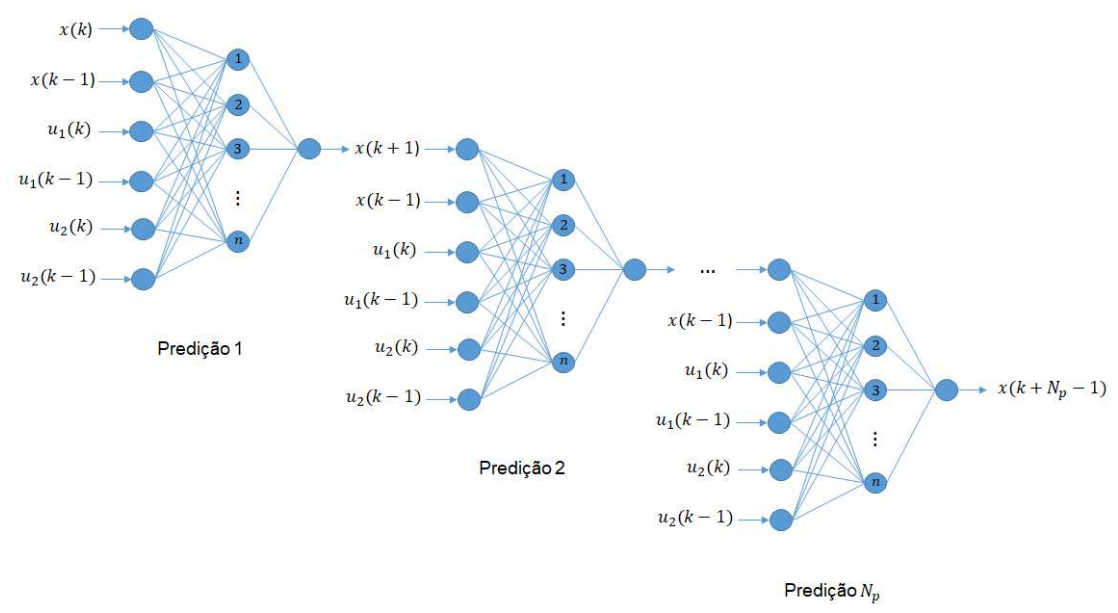

Figura 9: Estrutura do modelo de redes neurais recursivo para implementação do NN-NMPC.

A saída da primeira predicao $x(k+1)$ é então usada como entrada do modelo para a próxima predição $x(k+2)$, e assim por diante, até $x\left(k+N_{p}-1\right)$. Com esse método recursivo, múltiplas saídas podem ser preditas $N_{p}$ passos a frente, conforme Figura 9. As saídas preditas passam então por uma otimização para cálculo de $N_{c}$ ações de controle presentes e futuras. 


\section{3. \\ Metodologia}

Neste capítulo, é apresentada a metodologia para a simulação estática e dinâmica de uma coluna de destilação despropanizadora, para a implementação da comunicação entre o Aspen e o Matlab, para a obtenção dos dados do sistema, para a modelagem e identificação dos parâmetros dos modelos, para a sintonia dos controladores NMPC e testes de performance dos controladores PID e NMPC.

\section{1. Simulação estática}

A coluna de destilação despropanizadora estudada neste trabalho é baseada no procedimento para simulação de coluna de destilação despropanizadora simulada no software Aspen Plus ${ }^{\circledR}$ de Luyben (2006).

Para a realização da simulação estática, são especificados os parâmetros da coluna de destilação (número de pratos, tipo de condensador e refervedor, pressão e perfil de temperatura) e as propriedades das correntes (taxa de vazão, composição, temperatura e pressão). Também são definidos os componentes químicos envolvidos na separação e é definido o pacote de propriedades físicas.

A relação de equilíbrio líquido-vapor para a maioria dos sistemas de hidrocarbonetos é bem descrita pela correlação de Chao-Seader. Portanto, esta foi utilizada. A coluna simulada possui 32 pratos, em que a entrada da corrente de alimentação é realizada no prato 16 e as saídas dos produtos destilado e de fundo nos pratos 1 e 32 , respectivamente. O prato 1 representa o condensador e o prato 32 representa o refervedor.

As condições da corrente de alimentação $\mathrm{F}$ são $1 \mathrm{kmol} / \mathrm{s}, 322 \mathrm{~K}$ e $20 \mathrm{~atm}$. A composição da alimentação é de $40 \%$ de propano (C3) e $60 \%$ de butano (C4). De forma a maximizar o lucro, considera-se 3\% de C4 como impureza no topo em consonância com a especificação máxima exigida pela Resolução ANP №16, de 17.6.2008 apresentada na seção 2.1.1. Para atingir as especificações desejadas dos produtos, faz-se o uso da função "Design Specs" do Aspen Plus ${ }^{\circledR}$, em que é especificado o valor desejado da variável controlada e a variável a ser manipulada e a simulação tenta ajustar a variável manipulada de tal maneira que o valor especificado da variável controlada seja alcançado. Deseja-se convergir a especificação da composição do destilado ajustando a vazão molar do destilado. 
Então, o produto de topo foi especificado com $97 \%$ de propano, tendo então o produto de fundo com $1 \%$ de propano como resultado.

O condensador é especificado com a pressão interna de 16,8 atm e a coluna é especificada com uma queda de pressão de 0,0068 atm ao longo de cada prato. O condensador considerado é total e o refervedor é tipo Kettle. A temperatura do topo da coluna é de $325,08 \mathrm{~K}$, a do fundo é de $367,8 \mathrm{~K}$ e a do prato sensível 9 é 339,46 K.

Do condensador, saem duas vazões de correntes sendo estas o produto destilado a $0,392 \mathrm{kmol} / \mathrm{s}$ e o refluxo a $1,3 \mathrm{kmol} / \mathrm{s}$, ambas especificadas na coluna C1. Do refervedor, parte é vaporizada retornando para a coluna e o restante é retirado como produto de fundo a $0,608 \mathrm{kmol} / \mathrm{s}$. O calor no condensador e no refervedor é de -2.2105.283,8 Watt e 26.832.199,4 Watt, respectivamente.

O diagrama de processo utilizado é apresentado na Figura 10.

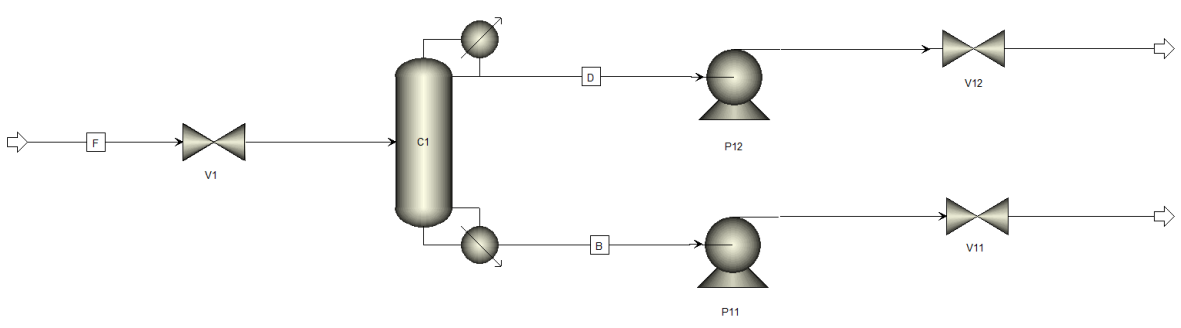

Figura 10: Simulação da coluna de destilação em Aspen Plus ${ }^{\circledR}$.

\section{2. \\ Simulação dinâmica}

Para realizar uma simulação dinâmica movida a pressão após a conclusão da simulação em estado estacionário, são dimensionados os equipamentos (coluna, válvulas de controle e bombas) e especificados os parâmetros operacionais.

Todas as válvulas são especificadas com queda de pressão de 3 atm. Tal fato se justifica pois é crucial para a controlabilidade dinâmica o fornecimento de queda de pressão suficiente sobre uma válvula de controle nas condições de projeto com a válvula com abertura parcial (normalmente $50 \%$ ). Se a queda de pressão da válvula for muito pequena, a válvula pode saturar perdendo controlabilidade. As bombas P11 e P12 são especificadas com aumento de pressão de 6 atm.

Para dimensionar o vaso de refluxo e a base da coluna, é utilizada uma heurística de 5 min de hold up quando o vaso estiver $50 \%$ cheio, com base no 
líquido total que entra ou sai. O vaso de refluxo é dimensionado com 4,08 $\mathrm{m}$ de diâmetro e 8,16 m de comprimento e a base da coluna com 5,08 m de diâmetro e $10,16 \mathrm{~m}$ de comprimento. O comprimento da coluna é especificado em 21,96 m e o diâmetro em 5,3 m.

Com essas especificações, é possível passar a simulação estática no Aspen Plus ${ }^{\circledR}$ para o modo dinâmico movido a pressão no Aspen Plus Dynamic ${ }^{\circledR}$. Adicionamente, é necessário definir uma estrutura de controle regulatório e supervisório e efetuar a sintonia dos controladores, conforme descrito a seguir.

\subsection{1.}

\section{Estruturas de controle}

Antes de elaboração da estrutura de controle regulatório do um processo, são definidos os graus de liberdade existentes na simulação. Os graus de liberdade do sistema são associados às válvulas de controle, indicando as possíveis variáveis manipuladas:

- Vazão do produto de topo (D);

- Vazão do produto de fundo (B);

- Calor do condensador $\left(Q_{\text {cond }}\right)$;

- Calor do refervedor (Qret);

- Vazão de refluxo (R);

- Vazão de Alimentação (F).

Em seguida, são definidas as estruturas do controle regulatório da planta. Primeiramente, para garantir a segurança do processo, a pressão do topo da coluna é controlada manipulando-se o calor do condensador $Q_{\text {cond }}(P I C-1)$.

Em seguida, é realizado o controle de inventário do vaso do condensador, controlando o nível de líquido manipulando-se $D$ através da abertura da válvula V12 (LIC-1). O nível no vaso do refervedor é controlado manipulando-se B através

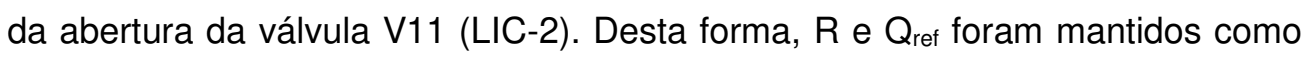
graus de liberdade.

Para controle da razão de refluxo e da temperatura do prato sensível, são incluídos dois controladores: o FIC-2 para controle da vazão de refluxo (R), manipulando-se a abertura da válvula Vr e o TIC-9 para controle da temperatura do prato sensível 9 manipulando-se o calor do refervedor $Q_{\text {ref. }}$

Adicionalmente, é incluído um controle de vazão na alimentação (FIC-1) para estudar o comportamento de distúrbios na entrada do sistema. 
A Tabela 5 apresenta a estrutura de controladores utilizados no controle regulatório e as respectivas variáveis controladas $(\mathrm{PV})$, variáveis manipuladas (OP) e o tipo de ação de controle.

Tabela 5: Estrutura do controle regulatório.

\begin{tabular}{|c|c|c|c|}
\hline Controle & PV & OP & ação \\
\hline FIC-1 & Vazão $F$ & $\mathrm{~V}_{1}$ & reversa \\
\hline FIC-2 & Vazão $\mathrm{R}$ & $\mathrm{V}_{\mathrm{R}}$ & reversa \\
\hline PIC-1 & P prato 1 & $\mathrm{Q}_{\text {cond }}$ & reversa \\
\hline LIC-1 & L prato1 & $\mathrm{V}_{12}$ & direta \\
\hline LIC-2 & L prato 32 & $\mathrm{V}_{11}$ & direta \\
\hline TIC-9 & T prato 9 & Qref & reversa \\
\hline
\end{tabular}

Para o controle de composição, são adotadas duas estratégias de controle em cascata: uma através de uma camada de controle preditivo não linear (NMPC) e outra utilizando-se controladores do tipo PID para fins de comparação.

O controlador multivariável NMPC manipula o setpoint dos controladores FIC-2 e TIC-9, em cascata, controlando-se a composição de butano no topo e a composição de propano no produto de fundo da coluna, conforme apresenta a Figura 11.

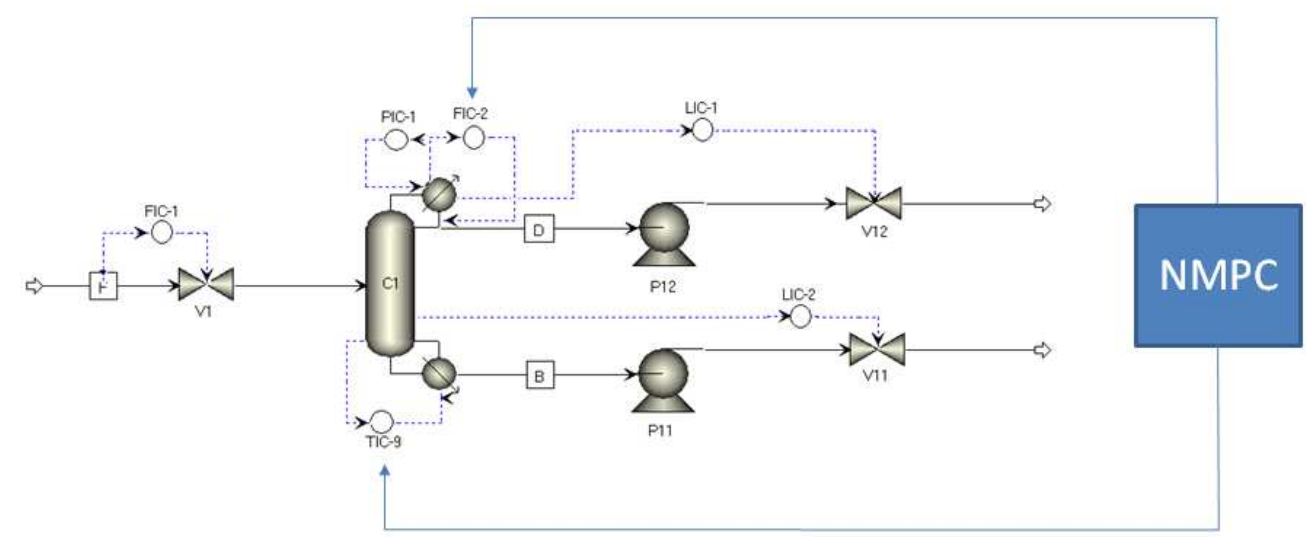

Figura 11: Simulação da coluna de destilação em Aspen Plus Dynamics ${ }^{\circledR}$ para simulação de controladores NMPC.

Na estratégia do NMPC são comparados a performance de dois modelos internos: modelos do tipo Hammerstein (H-NMPC) e do tipo Redes Neurais (NNNMPC). Uma descrição detalhada de cada modelo é apresentada no item 3.5.

Já na estratégia de controle PID, a composição de butano no produto de topo é controlada em cascata com o CC4D manipulando-se o setpoint do 
controlador FIC-2. E a composição de propano no produto de fundo é controlada em cascata pelo controlador CC3B manipulando-se o setpoint do TIC-9, conforme Figura 12.

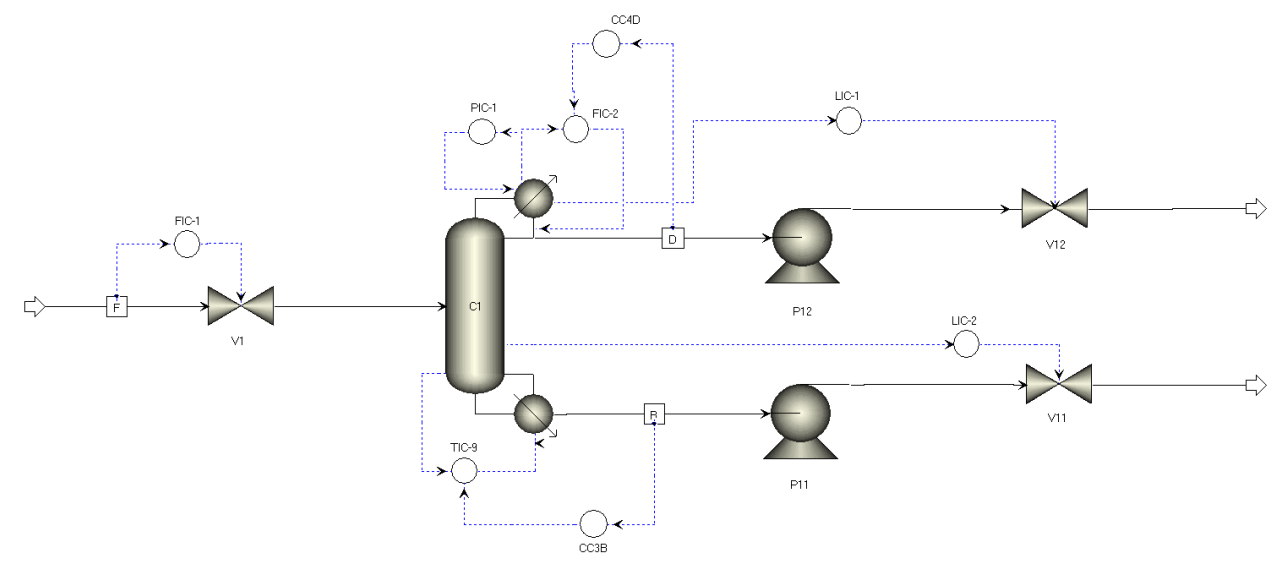

Figura 12: Simulação da coluna de destilação em Aspen Plus Dynamics ${ }^{\circledR}$ para simulação de controladores PID.

A Tabela 6 apresenta a estrutura dos controladores supervisórios da estratégia de controle NMPC e de controle PID.

Tabela 6: Estrutura dos controladores supervisórios da estratégia de controle NMPC e de controle PID.

\begin{tabular}{|c|c|c|c|c|}
\hline Estratégia & Controle & PV & OP & ação \\
\hline NMPC & $\begin{array}{c}\text { NMPC (com modelo interno } \\
\text { do tipo Hammerstein ou } \\
\text { tipo rede Neural) }\end{array}$ & C3B e C4D & $\begin{array}{c}\text { Setpoint do } \\
\text { FIC-2 e TIC-9 }\end{array}$ & - direta \\
\hline PID & CC3B & C3B & $\begin{array}{c}\text { Setpoint do } \\
\text { TIC-9 }\end{array}$ & reversa \\
\hline PID & CC4D & C4D & $\begin{array}{c}\text { Setpoint do } \\
\text { FIC-2 }\end{array}$ & \\
\hline
\end{tabular}

\subsection{2.}

\section{Sintonia dos controladores PID}

O controlador $\mathrm{P}$ é a forma mais simples de controle, de fácil sintonia e fornece o controle mais robusto (ou seja, estável). Este fornece uma resposta rápida a distúrbios e alterações de setpoint, mas está sujeito a offset. Este é adequado para aplicações dinâmicas, como controle de nível. O controlador PI é a configuração mais comum do controlador PID na industria. Ele fornece a resposta inicial rápida de um controlador $\mathrm{P}$, e corrige os resultados resultantes do 
controle P. O uso de dois parâmetros torna essa configuração relativamente fácil de ajustar. Controladores PID permite termos Proporcionais e Integrais mais

agressivos sem introduzir overshoot. É bom para uso em processos estáveis e/ou processos que respondem lentamente ou têm pouco ou nenhum ruído. O ponto negativo do controlador PID é sua complexidade adicional e o aumento da vibração no sinal de saída do controlador. Portanto, são utilizados controladores $\mathrm{P}$ para controles de nível e PI para os demais controles de forma a minimizar overshoot. É feita a sintonia dos controladores PID de acordo com Luyben (2006).

O controle de pressão é configurado automaticamente quando o Aspen Plus Dynamic ${ }^{\circledR}$ foi inicializado com os parâmetros de sintonia de ganho em $20 \mathrm{e}$ tempo integral em 12 min, os quais costumam funcionar bem para colunas de destilação. O controle é configurado para ação reversa, porque quando a pressão aumenta, o sinal de saída do controlador diminui.

Para controle de nível do refervedor, a ação de controle utilizada é direta porque quando o nível aumenta, a abertura da válvula aumenta para remover produto de fundo. Como é desejado controle proporcional apenas, o ganho é configurado para 2 e o tempo integral é configurado para um valor grande de 9999 min. Para o controle de nível do tanque de refluxo, a mesma configuração é realizada e um controlador de ação direta proporcional é especificado.

Para o controle de vazão da alimentação, são utilizados valores convencionais para sintonia de vazão ( $\mathrm{k}_{\mathrm{p}}=0,5$ e $\left.\tau_{i}=0.3 \mathrm{~min}\right)$ e ação reversa. Como um controle de vazão é tipicamente rápido, a adição de um filtro auxilia o integrador numérico. Então, foi inserido um filtro de constante de tempo de 0,1 $\min$.

A sintonia de controles de temperatura e composição é mais complicada que simplesmente usar a heurística como foi usado para controles de vazão e nível. O método de sintonia utilizado foi o método de Tyres-Luyben no Aspen Plus Dynamic $^{\circledR}$.

\subsection{Comunicação ASPEN/MATLAB}

A comunicação entre a simulação da coluna despropanizadora no Aspen Plus Dynamic ${ }^{\circledR}$ e o controle programado em Matlab é feita por Simulink utilizando o bloco AMSimulation, mostrado na Figura 13. 

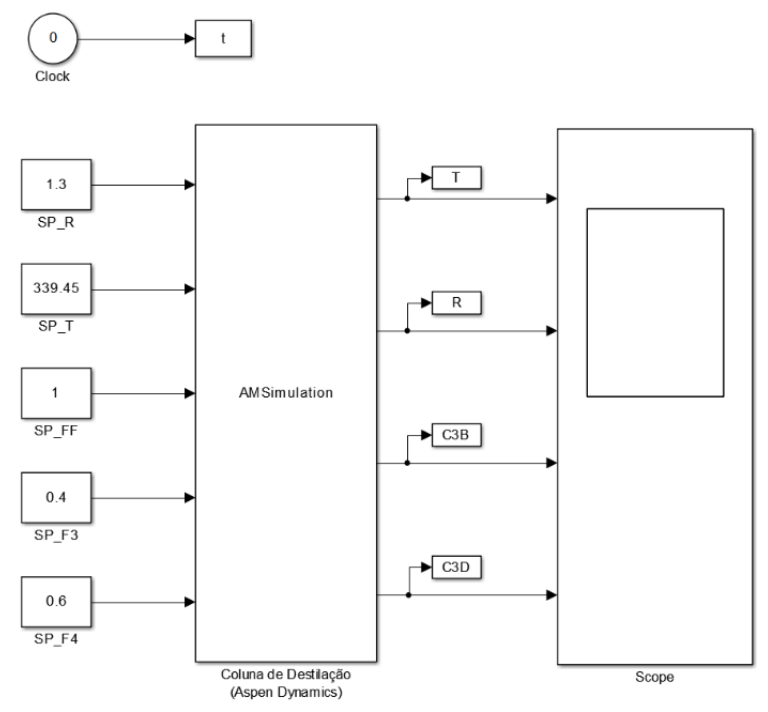

Figura 139: Diagrama de blocos do Simulink.

O sistema proposto possui duas entradas e duas saídas, sendo as variáveis manipuladas:

- R: Setpoint do Controle de Refluxo interno, FIC-2;

- T: Setpoint do Controle de Temperatura do prato sensível 9, TIC-9;

e as variáveis controladas:

- C3B: fração molar de propano no fundo da coluna;

- C4D: fração molar de butano no topo da coluna.

Também foram incluídas 3 entradas referente à composição (2 componentes) e vazão da alimentação de modo a estudar os distúrbios causados por estas variáveis.

- FF: Vazão da alimentação;

- C3: Componente propano na alimentação;

- C4: Componente iso-butano na alimentação;

Quando não há distúrbio nas simulações, estas 3 entrada são mantidas constantes com os valores do estado estacionário, alterando somente R e T. 


\section{4 . \\ Obtenção dos dados}

Primeiramente, é testada uma ampla faixa de operação para as variáveis $\mathrm{R}$ e $\mathrm{T}$, de forma a conhecer os limites operacionais da simulação. O ponto de operação de projeto é $R=1,3 \mathrm{kmol} / \mathrm{h}$ e $T=339,5 \mathrm{~K}$. A faixa de operação utilizada de $R$ é de 0,1 a $2 \mathrm{kmol} / \mathrm{s}$, com degraus de $0,1 \mathrm{kmol} / \mathrm{s}$, e a faixa empregada na variável T é de 333 a 342,5 K, com degraus de 0,5 K. Observa-se que nesta faixa, não há restrições físicas do sistema (como vazões de refluxo e refervimento nulas), não havendo erro de convergência na simulação.

É realizada uma varredura de modo a percorrer toda a faixa de temperatura para cada vazão de refluxo e vice-versa, num período de amostragem de $\Delta t=$ $0,1 \mathrm{~h}$, em que cada degrau tem um tempo de $10 \mathrm{~h}$ para garantir tempo de assentamento após um degrau, totalizando 152 mil pontos.

\section{5. \\ Modelagem e identificação dos parâmetros dos modelos}

Nesta seção, é apresentada a metodologia para a modelagem do sistema e a identificação dos parâmetros dos modelos de Hammerstein e Redes Neurais.

\subsection{1. \\ Modelo de Hammerstein \\ Esta seção apresenta a metodologia para a modelagem do bloco estacionário não-linear e do bloco dinâmico linear, identificação dos parâmetros do modelo e aplicação do MHE.}

\subsubsection{1.}

Modelo estacionário não-linear polinomial

O modelo estacionário não-linear polinomial é modelado a partir da modificação da função polinomial que descreve o comportamento das variáveis controladas em função das entradas e do instante $k$, como visto na Seção 2.4.3.1.

Assim, dado um sistema que possua 2 entradas $\left(u_{1}\right.$ e $\left.u_{2}\right)$, a variável manipulada é descrita por uma função polinomial não-linear $\phi_{m o d}$, em função do vetor de entradas $u=\left[u_{1}, u_{2}\right]^{T}$, com $p$ coeficientes, a qual é decomposta em $p$ funções $\left\{\phi_{1}(u), \phi_{2}(u), \ldots, \phi_{p}(u)\right\}$ conforme a Equação (101) 


$$
\phi(u, k)=\left[\begin{array}{c}
\phi_{1}\left(u_{1}(k)\right) \\
\phi_{2}\left(u_{1}(k)\right) \\
\vdots \\
\phi_{p}\left(u_{1}(k)\right) \\
\vdots \\
\phi_{1}\left(u_{2}(k)\right) \\
\phi_{2}\left(u_{2}(k)\right) \\
\vdots \\
\phi_{p}\left(u_{2}(k)\right)
\end{array}\right] \in \mathbb{R}^{2 . p \times 1} .
$$

A análise do efeito simultâneo das variáveis manipuladas $u_{1}=\mathrm{T}$ e $u_{2}=\mathrm{R}$ em cada uma das variáveis controladas $x_{1}=$ C3B e $x_{2}=$ C4D consiste na região de controle em que R varia de $0,1 \mathrm{a} 2 \mathrm{kmol} / \mathrm{s}$, com degraus de $0,1 \mathrm{kmol} / \mathrm{s}$, e T varia de 333 a $342,5 \mathrm{~K}$, com degraus de $0,5 \mathrm{~K}$.

Os dados são analisados no software Matlab $^{\circledR}$ para identificar os parâmetros do modelo estacionário não-linear da coluna de destilação, de forma a encontrar uma função polinomial da superfície gerada para cada uma das variáveis controladas em função das entradas. Para isso, é utilizado o aplicativo Curve Fitting do Matlab ${ }^{\circledR}$ para ajustar os dados para obter a função polinomial que descreve a superfície gerada.

\subsubsection{2.}

\section{Modelo dinâmico linear em espaço de estado}

Considerando a dinâmica de ambas as respostas sendo de primeira ordem, um modelo típico em espaço de estado utilizado pode ser representado a partir das equações

$$
\begin{aligned}
& \frac{\partial x_{1}}{\partial t} \tau_{1}+x_{1}=k p_{1} u \\
& \frac{\partial x_{2}}{\partial t} \tau_{2}+x_{2}=k p_{2} u \\
& y_{1}=x_{1} \\
& y_{2}=x_{2},
\end{aligned}
$$

em que $\tau$ é a constante de tempo e $k p$ é o ganho estático. O ganho estático indica a sensibilidade do comportamento estático de uma saída frente ao degrau.

Isolando os termos $\frac{\partial x_{1}}{\partial t} \mathrm{e} \frac{\partial x_{2}}{\partial t}$, obtem-se as equações para a formulação do espaço de estado 
$\frac{\partial x}{\partial t}=\left[\begin{array}{cc}-\frac{1}{\tau_{1}} & 0 \\ 0 & -\frac{1}{\tau_{2}}\end{array}\right] x+\left[\begin{array}{c}\frac{k p_{1}}{\tau_{1}} \\ \frac{k p_{2}}{\tau_{2}}\end{array}\right] u$

$y=\left[\begin{array}{ll}1 & 0 \\ 0 & 1\end{array}\right] x$

Para obter a formulação do modelo modificado em espaço de estado conforme a Equação abaixo

$\frac{\partial x}{\partial t}=A x+B_{\text {mod }} \phi_{\text {mod }}$

$y=C x$

e substituído o termo $\phi_{m o d}$ obtido na modelagem estacionária e a matriz $B$ é modificada repetindo os termos $p$ vezes obtendo-se então a matriz $B_{\text {mod }}$ conforme Equação

$B_{\text {mod }}=\left[\begin{array}{ccc}\frac{k p_{1,1}}{\tau_{1}} & \ldots & \frac{k p_{1, p}}{\tau_{1}} \\ \frac{k p_{2,1}}{\tau_{2}} & \ldots & \frac{k p_{2, p}}{\tau_{2}}\end{array}\right]$.

3.5.1.3.

Identificação dos parâmetros do modelo

A identificação do modelo em espaço de estado é feita de forma a estimar os parâmetros $\tau_{1}, \tau_{2}, k p_{1,1}$ a $k p_{1,13}$ e $k p_{2,1}$ a $k p_{2,13}$ do modelo modificado. Esta é feita através do algoritmo Nelder-Mead disponível na função fminsearch do Matlab $^{\circledR}$, minimizando-se a soma do erro quadrático entre a curva com os dados experimentais do sistema e a curva obtida pelo modelo com valores dos parâmetros calculados pela função a cada interação, até obter um ajuste satisfatório.

\subsubsection{4.}

\section{Estimador de Horizonte Móvel}

O MHE é incluído de forma a atualizar o modelo interno do controlador preditivo com a estimação dos parâmetros $x_{1}=\mathrm{C} 3 \mathrm{~B}$ e $x_{2}=\mathrm{C} 4 \mathrm{D}$, frente a ruídos de medição. Como as variáveis $x_{1}$ e $x_{2}$ são referentes a frações molares, seus valores são restritos à faixa de valores entre 0 e 1. 
A sintonia do MHE é feita a partir das recomendações de sintonia feitas por Rao e Rawlings (2002), distutidas na Seção 2.4.3.4. Os parâmetros de sintonia para reconciliar o modelo com as medições do processo são:

- Q: matriz que indica a incerteza no modelo,

- R: matriz que indica a medida de confiança nos sensores do processo,

- $\quad$ П: matriz que fornece uma medida de confiança do estado inicial.

\subsection{2.}

\section{Modelo por Redes Neurais}

Esta seção apresenta a metodologia para a modelagem do modelo de redes neurais, do modelo interno do controlador NN-NMPC o cálculo da função objetivo.

\subsubsection{1. \\ Modelo interno do controlador NN-NMPC}

O modelo proposto tem como entradas da rede neural, com apenas uma camada escondida com $n$ neurônios, a variável de estado do sistema $(x)$ e duas variáveis de controle $\left(u_{1}\right.$ e $\left.u_{2}\right)$ no instante atual $(k)$ conforme a Equação (111)

$x(k+1)=f\left(x(k), u_{1}(k), u_{2}(k)\right)$.

A saída da primeira predicao $x(k+1)$ é então usada como entrada do modelo para a próxima predição $x(k+2)$, e assim por diante, até $x\left(k+N_{p}-1\right)$. Com esse método recursivo, múltiplas saídas podem ser preditas $N_{p}$ passos a frente, conforme Figura 14. As saídas preditas passam então por uma otimização para cálculo de $N_{c}$ ações de controle presentes e futuras. 


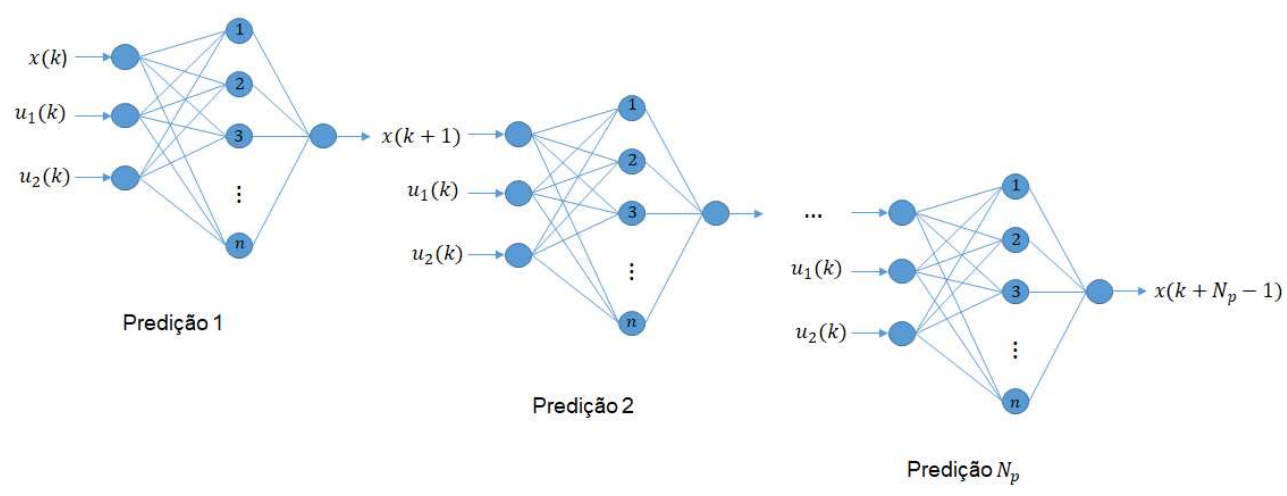

Figura 14: Estrutura do modelo de redes neurais recursivo proposta para implementação do NN-NMPC.

Assim, dado um sistema que possua $m$ entradas $(u), q$ saídas $(y)$ e $n$ variáveis de estado $(x)$, considera-se o vetor de estado para o horizonte de predição até $N_{p}$ passos à frente por

$X=\left[\begin{array}{c}x(k) \\ x(k+1) \\ \vdots \\ x\left(k+N_{p}-1\right)\end{array}\right] \in \mathbb{R}^{n \cdot N_{p} \times 1}$

o vetor das variáveis de saída preditas para o horizonte de predição até $N_{p}$ passos à frente por

$Y=\left[\begin{array}{c}y(k) \\ y(k+1) \\ \vdots \\ y\left(k+N_{p}-1\right)\end{array}\right] \in \mathbb{R}^{q \cdot N_{p} \times 1}$

e o vetor de ações de controle para um horizonte de controle até $N_{c}$ passos à frente por

$U=\left[\begin{array}{c}u(k) \\ u(k+1) \\ \vdots \\ u\left(k+N_{c}-1\right)\end{array}\right] \in \mathbb{R}^{m \cdot N_{c} \times 1}$

A variável manipulada pode então ser descrita por uma função não-linear por redes neurais conforme Equação 115 


$$
Y=\left[\begin{array}{c}
f\left(\sum_{i=1}^{n}\left(g\left(\sum_{j=1}^{m} U_{1} \cdot I W_{i, j}+X_{1} \cdot I W_{i, j+1}-\mathrm{LB}_{\mathrm{i}}\right) L W_{i}-O B\right)\right) \\
f\left(\sum_{i=1}^{n}\left(g\left(\sum_{j=1}^{m} U_{2} \cdot I W_{i, j}+X_{2} \cdot I W_{i, j+1}-\mathrm{LB}_{\mathrm{i}}\right) L W_{i}-O B\right)\right) \\
\vdots \\
f\left(\sum_{i=1}^{n}\left(g\left(\sum_{j=1}^{m} U_{N_{c}} \cdot I W_{i, j}+X_{N_{c}} \cdot I W_{i, j+1}-\mathrm{LB}_{\mathrm{i}}\right) L W_{i}-O B\right)\right) \\
f\left(\sum_{i=1}^{n}\left(g\left(\sum_{j=1}^{m} U_{N_{c}} \cdot I W_{i, j}+X_{N_{c}+1} \cdot I W_{i, j+1}-\mathrm{LB}_{\mathrm{i}}\right) L W_{i}-O B\right)\right) \\
\vdots \\
f\left(\sum_{i=1}^{n}\left(g\left(\sum_{j=1}^{m} U_{N_{c}} \cdot I W_{i, j}+X_{N_{p}} \cdot I W_{i, j+1}-\mathrm{LB}_{\mathrm{i}}\right) L W_{i}-O B\right)\right)
\end{array}\right]
$$

em que

$n$ é o número de neurônios,

$m$ é o número de entradas do sistema $(u)$,

$m+1$ é o número de entradas da rede,

IW é a matriz de pesos da entrada da rede $\in \mathbb{R}^{\mathrm{n} \times \mathrm{m}}$,

$L W$ é o vetor de pesos da camada escondida $\in \mathbb{R}^{n \times 1}$,

LB é o vetor bias da camada escondida $\in \mathbb{R}^{n \times 1}$,

OB é o escalar bias da saída,

$g$ é a função de ativação da camada escondida,

$f$ é a função de ativação da saída.

\subsubsection{2.}

\section{Função objetivo para modelo de Redes Neurais}

A função objetivo proposta por Wang (2009) discutida na Seção 2.3.3. é dada por

$J=0.5\left(Y^{s p}-Y\right)^{T} Q_{Y}\left(Y^{s p}-Y\right)+0.5 \Delta U^{T} Q_{\Delta U} \Delta U$,

em que

$\Delta U=C_{2}^{-1}\left(U-C_{1} u(k-1)\right)$ 
$\begin{aligned} C_{1} & =\left[\begin{array}{c}I_{m \times m} \\ I_{m \times m} \\ \vdots \\ I_{m \times m}\end{array}\right] \in \mathbb{R}^{m N_{c} \times m} \\ C_{2} & =\left[\begin{array}{llll}I_{m \times m} & 0_{m \times m} & \cdots & 0_{m \times m} \\ I_{m \times m} & I_{m \times m} & \cdots & 0_{m \times m} \\ \vdots & \vdots & \ddots & 0_{m \times m} \\ I_{m \times m} & I_{m \times m} & \cdots & I_{m \times m}\end{array}\right] \in \mathbb{R}^{m . N_{c} \times m . N_{c}} .\end{aligned}$

Substituindo a Equação (106) na Equação (105), temos

$J=\left(Y^{s p}-Y\right)^{T} Q_{Y}\left(Y^{s p}-Y\right)+\left[C_{2}^{-1}\left(U-C_{1} u(k-1)\right)\right]^{T} Q_{\Delta U}\left[C_{2}^{-1}\left(U-C_{1} u(k-1)\right)\right]$.

A função objetivo está sujeita às restrições descritas pelas desigualdades

$$
\begin{aligned}
& {\left[\begin{array}{c}
-I_{m \times N_{c}} \\
I_{m \times N_{c}} \\
-C_{2}^{-1} \\
C_{2}^{-1}
\end{array}\right] U \leq\left[\begin{array}{c}
-U^{\min } \\
U^{\max } \\
-\Delta U^{\min }-C_{2}^{-1} C_{1} u(k-1) \\
-\Delta U^{m i n}-C_{2}^{-1} C_{1} u(k-1)
\end{array}\right]} \\
& {\left[\begin{array}{c}
-I_{q \times N_{p}} \\
I_{q \times N_{p}}
\end{array}\right] Y+\left[\begin{array}{c}
Y^{\min } \\
-Y^{\max }
\end{array}\right] \leq 0 .}
\end{aligned}
$$

Para obter a função objetivo para o modelo de Redes Neurais, a saída predita dada pela Equação (123) é substituída na Equação (120)

$$
Y=\left[\begin{array}{c}
f\left(\sum_{i=1}^{n}\left(g\left(\sum_{j=1}^{m} U_{1} \cdot I W_{i, j}+X_{1} \cdot I W_{i, j+1}-\mathrm{LB}_{\mathrm{i}}\right) L W_{i}-O B\right)\right) \\
f\left(\sum_{i=1}^{n}\left(g\left(\sum_{j=1}^{m} U_{2} \cdot I W_{i, j}+X_{2} \cdot I W_{i, j+1}-\mathrm{LB}_{\mathrm{i}}\right) L W_{i}-O B\right)\right) \\
\vdots \\
f\left(\sum_{i=1}^{n}\left(g\left(\sum_{j=1}^{m} U_{N_{c}} \cdot I W_{i, j}+X_{N_{c}} \cdot I W_{i, j+1}-\mathrm{LB}_{\mathrm{i}}\right) L W_{i}-O B\right)\right) \\
f\left(\sum_{i=1}^{n}\left(g\left(\sum_{j=1}^{m} U_{N_{c}} \cdot I W_{i, j}+X_{N_{c}+1} \cdot I W_{i, j+1}-\mathrm{LB}_{\mathrm{i}}\right) L W_{i}-O B\right)\right) \\
\vdots \\
f\left(\sum_{i=1}^{n}\left(g\left(\sum_{j=1}^{m} U_{N_{c}} \cdot I W_{i, j}+X_{N_{p}} \cdot I W_{i, j+1}-\mathrm{LB}_{\mathrm{i}}\right) L W_{i}-O B\right)\right)
\end{array}\right] .
$$


Conforme discutido anteriormente, raramente a função não-linear que descreve o estado num instante a frente está disponível na forma algébrica. Em vez disso, a função é vista abstratamente como a solução numérica com uma condição inicial.

Devido à complexidade do procedimento de otimização, para o modelo de redes neurais, esta deve ser resolvida de forma numérica com uma condição inicial $X_{1}$, de forma a encontrar a sequência de ações de controle que minimiza a função objetivo.

A Equação (95) é substituída na Equação (75) de forma a fornecer as restrições não-lineares de forma numérica. A Equação (94) fornece as restrições lineares também de forma numérica.

A estrutura proposta do NN-NMPC é observada na Figura 15, em que o NN-NMPC recebe o valor medido da variável de saída $y_{m}$ o qual calcula o melhor sinal de entrada $u$ para a camada de controle regulatório da planta.

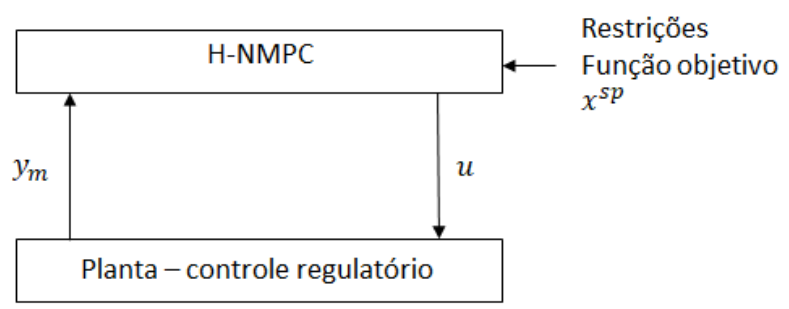

Figura 1510: Estrutura do NN-NMPC.

\subsubsection{3. Identificação dos parâmetros do modelo}

Os dados são analisados no software Matlab $^{\circledR}$ para identificar os parâmetros da não-linearidade dinâmica do modelo da coluna de destilação, de forma a encontrar os parâmetros da rede neural para cada uma das saídas. $O$ treinamento da rede é feito utilizando a função newff e o algoritmo de treinamento utilizado é o Levenberg Marquadt (train/m). As entradas da rede são normalizadas entre -1 e 1, a função de ativação da camada escondida foi a tansig e a função de ativação da saída é a purelin.

A rede neural possui como entrada para treinamento os sinais de entrada do sistema $u_{1}=\mathrm{T}$ e $u_{2}=\mathrm{R}$, nas faixas faixa $0,9 \leq \mathrm{R} \leq 1,4 \mathrm{kmol} / \mathrm{s}$ e $335 \leq \mathrm{T} \leq$ $340 \mathrm{~K}$, e o estado no instante atual $k$. A rede possui como saída predita uma predição do estado no instante $k+1$. Uma rede foi treinada para cada variável de estado do sistema, em que $70 \%$ dos dados são usados para o treino da rede e 
$30 \%$ usado para o teste da rede. O referido modelo é implementado como mostrado na Figura 16.

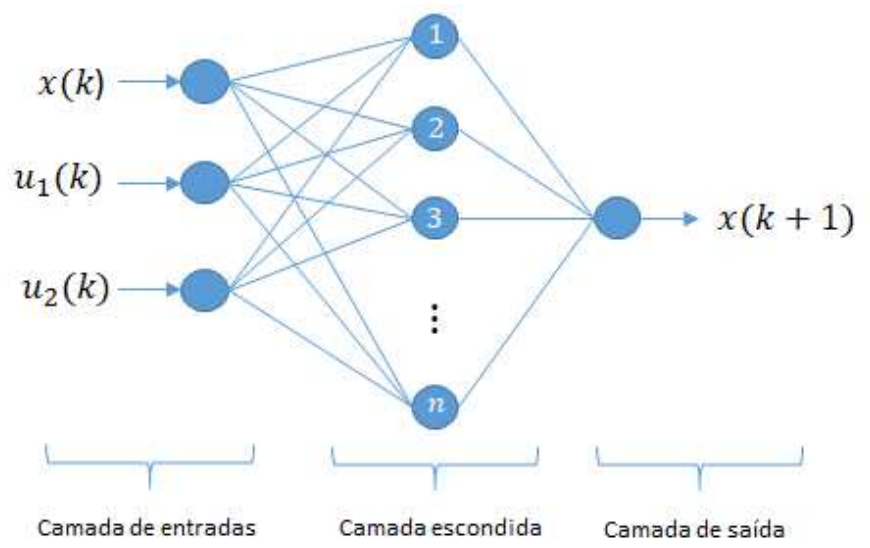

Figura 16: Modelo de redes neurais.

Assim, são testadas redes de 1 a 10 neurônios na camada escondida de forma a encontrar o melhor modelo.

Após definir o modelo, a saída da primeira predicao $x(k+1)$ é usada como entrada do modelo para a próxima predição $x(k+2)$ e assim por diante, até $x\left(k+N_{p}-1\right)$. Com esse método iterativo, múltiplas saídas podem ser preditas $N_{p}$ passos a frente. Assim, é aplicado o método iterativo para o modelo de forma a prever $N_{p}=40$ passos a frente após um degrau.

\section{6.}

\section{Sintonia dos controladores NMPC}

A sintonia do controle preditivo é feita a partir das recomendações de sintonia feitas por Seborg et al. (2011), distutidas na Seção 2.3.4. Os parâmetros de sintonia são:

- $Q_{\Delta U}$ : matriz de ponderação de entrada, a qual permite que as varáveis manipuladas sejam ponderadas de acordo com sua importância relativa

- $N_{C}$ : Horizonte de controle

- $Q_{Y}$ : matriz de ponderação de saída da função custo, a qual permite que as variáveis controladas sejam ponderadas de acordo com sua importância relativa. 


\section{7. \\ Testes de performance dos controladores}

Os testes são realizados de forma a comparar a performance das 3 estratégias de controle estudadas: H-NMPC, NN-NMPC e controle PID tradicional. Os testes são feitos para verificar o rastreamento da trajetória de referência, o desacoplamento das variáveis controladas e a rejeição de distúrbios na vazão e composição da alimentação.

Os testes de rastreamento para controle dual das trajetórias de referências das composições dos produtos de topo e de fundo da coluna despropanizora são feitos variando-se a amplitude em forma de degrau, conforme valores de referência apresentados na Tabela 7. O teste de desacoplamento das variáveis controladas é feito mantendo o valor de referência constante para C4D, dando um degrau para C3B.

Tabela 7: Valores de referência das variáveis controladas para teste de rastreamento e desacoplamento destas.

\begin{tabular}{|c|c|c|}
\cline { 2 - 3 } \multicolumn{1}{c|}{} & C3B & C4D \\
\hline 1 & 0.0788 & 0.0230 \\
\hline 2 & 0.0836 & 0.0212 \\
\hline 3 & 0.0884 & 0.0196 \\
\hline 4 & 0.0980 & 0.0166 \\
\hline 5 & 0.1030 & 0.0153 \\
\hline 6 & 0.1100 & 0.0153 \\
\hline 7 & 0.0932 & 0.0180 \\
\hline
\end{tabular}

Os testes de distúrbio da corrente de alimentação são feitos de forma a observar a controlabilidade dos controladores ao tentar manter o setpoint dentro da especificação. Para o teste de distúrbio na vazão de alimentação, é aplicado um degrau no valor de $10 \%$ variando-se a amplitude da vazão em forma de degrau, alterando a vazão de $1 \mathrm{kmol} / \mathrm{s}$ para $1,1 \mathrm{kmol} / \mathrm{s}$. Para o teste de distúrbio na composição de alimentação, é aplicado um distúrbio de $10 \%$ na composição de propano da alimentação da coluna de destilação, variando-se a amplitude em forma de degrau, aumentando a fração molar de C3 de 0,4 para 0,44 e diminuindo a do C4 de 0,6 para 0,56.

Um sistema de controle é dito ser ótimo quando o índice de desempenho selecionado é minimizado. Essas métricas representam uma avaliação do funcionamento da planta, sendo a soma dos erros quadráticos (SSE), apresentada pela Equação (124) 
$S S E=\sum_{t=1}^{\infty} e_{t}^{2}$

o mais utilizado em sistemas com características pouco oscilatórias. Portanto, esta métrica é usada para avaliar o desempenho dos controladores nos testes de distúrbios. 


\section{Resultados e discussões}

Neste capítulo, são apresentados resultados e discussões para a implementação dos controladores NMPC, incluindo modelagem e identificação dos parâmetros dos modelos, sintonia dos controladores NMPC e testes de performance dos controladores PID e NMPC.

\section{1.}

\section{Obtenção dos dados}

Foi realizada uma varredura de modo a percorrer toda a faixa de temperatura para cada vazão de refluxo e vice-versa, num período de amostragem de $\Delta t=0,1 \mathrm{~h}$, em que cada degrau tem um tempo de $10 \mathrm{~h}$ para garantir tempo de assentamento após um degrau. A Figura 17 apresenta os setpoints das variáveis manipuladas $\mathrm{R}$ e $\mathrm{T}$ e as respostas das variáveis controladas C3B e C4D dos dados obtidos.
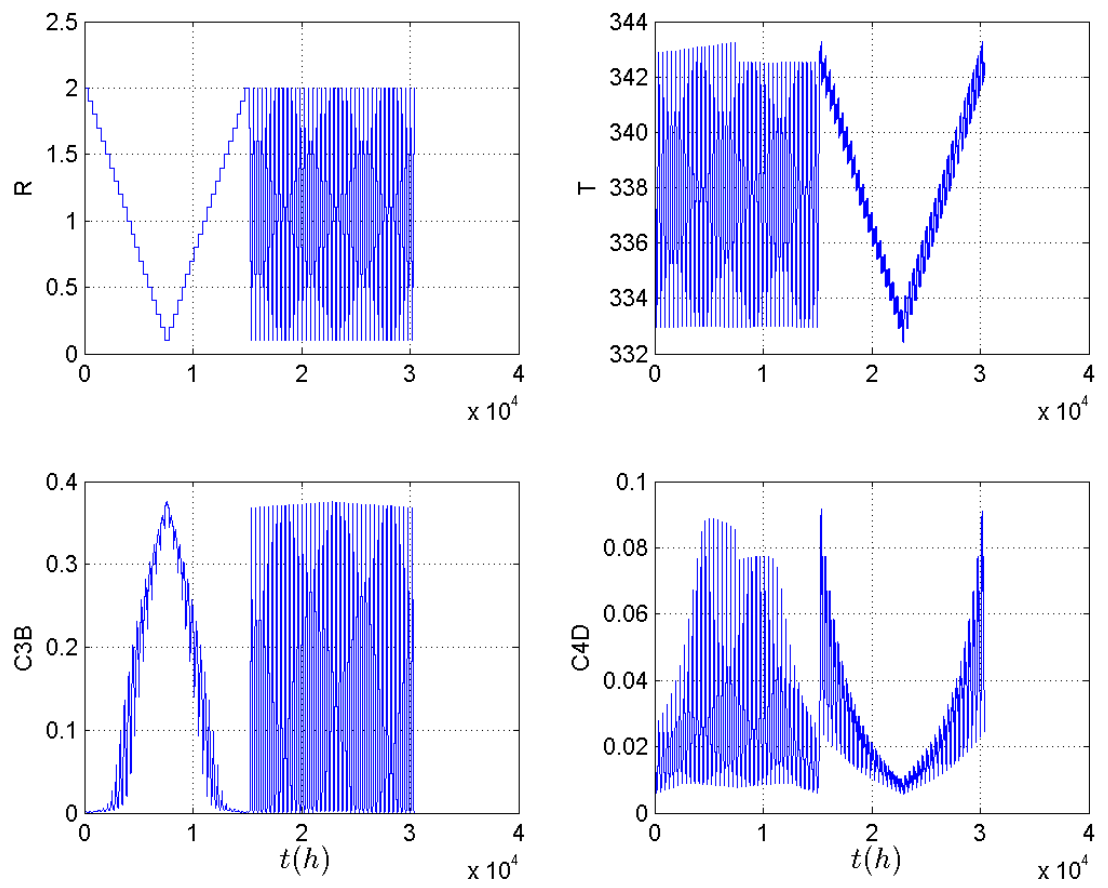

Figura 1711: Gráficos dos setpoints das variáveis manipuladas $\mathrm{R}$ e $\mathrm{T}$ e as respostas das variáveis controladas C3B e C4D. 


\section{2.}

\section{Modelagem e identificação dos parâmetros dos modelos}

Nesta seção, é feita a modelagem do sistema e a identificação dos parâmetros dos modelos de Hammerstein e Redes Neurais, de forma a se obter valores numéricos adequados aos parâmetros desconhecidos.

\subsection{1.}

\section{Modelo de Hammerstein}

Esta seção apresenta a modelagem do bloco estacionário não-linear e do bloco dinâmico linear, identificação dos parâmetros do modelo e aplicação do MHE.

\subsubsection{1.}

\section{Modelo estacionário não-linear polinomial}

Foi analisado o efeito simultâneo das variáveis manipuladas $u_{1}=\mathrm{T}$ e $u_{2}=$ $\mathrm{R}$, variando $\mathrm{R}$ de 0,1 a $2 \mathrm{kmol} / \mathrm{s}$, com degraus de $0,1 \mathrm{kmol} / \mathrm{s}$ e variando $\mathrm{T}$ de 333 a $342,5 \mathrm{~K}$, com degraus de $0,5 \mathrm{~K}$, em cada uma das variáveis controladas C3B e C4D.

As Figuras 18 e 19 mostram as superfícies modeladas para as saídas C3B e C4D, respectivamente.

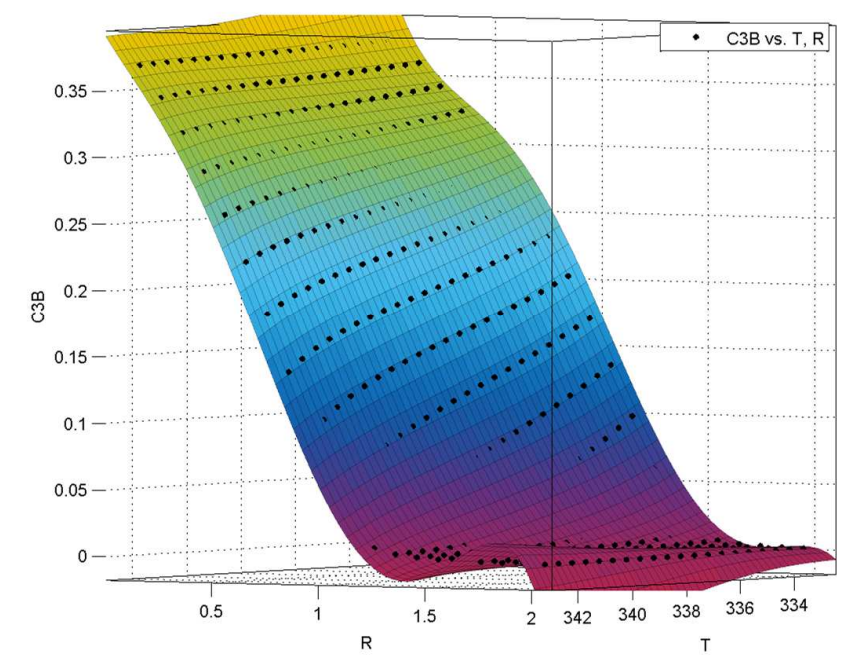

Figura 1812: Superfície para a saída C3B em função de T e R. 


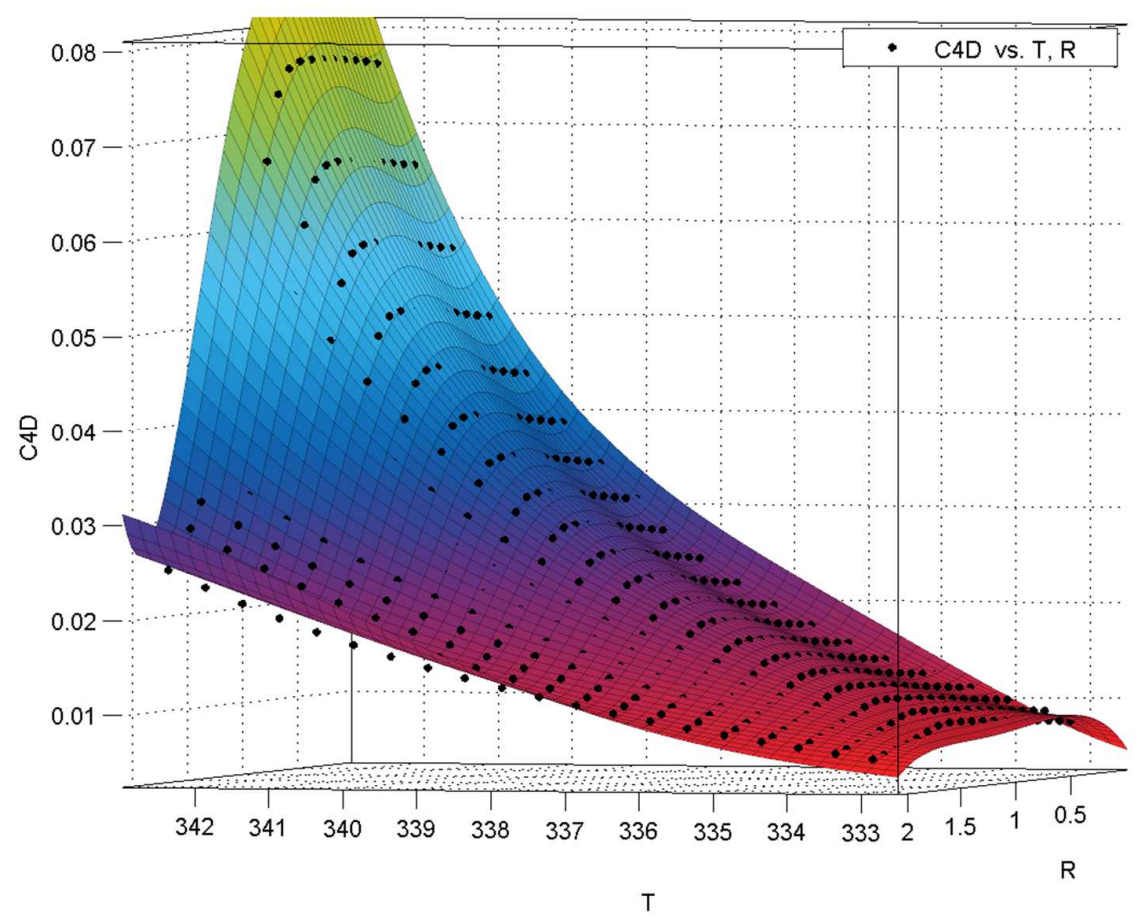

Figura 19: Superfície para a saída C4D em função de T e R.

Como resultado, uma equação polinomial de grau $3 \times 4$ foi ajustada aos dados experimentais de forma a descrever bem ambas as superfícies conforme a seguir

$$
\begin{array}{r}
y_{s s}\left(u_{1}, u_{2}\right)=b_{1} u_{1}+b_{2} u_{2}+b_{3} u_{1}{ }^{2}+b_{4} u_{1} u_{2}+b_{5} u_{2}{ }^{2}+b_{6} u_{1}{ }^{3}+b_{7} u_{1}{ }^{2} u_{2}+ \\
b_{8} u_{1} u_{2}{ }^{2}+b_{9} u_{2}{ }^{3}+b_{10} u_{1}^{3} u_{2}+b_{11} u_{1}{ }^{2} u_{2}{ }^{2}+b_{12} u_{1} u_{2}{ }^{3}+b_{13} u_{2}{ }^{4}
\end{array}
$$

Os coeficientes das equações polinomiais para ambas as superficies são apresentados na Tabela 8. 
Tabela 8: Coeficientes das equações polinomiais 3x4 para C3B e C4D.

\begin{tabular}{|c|c|}
\hline $\boldsymbol{u}_{\mathbf{1}} \times \boldsymbol{u}_{\mathbf{2}} \times \boldsymbol{x}_{\mathbf{1}}$ & $\boldsymbol{u}_{\mathbf{1}} \times \boldsymbol{u}_{\mathbf{2}} \times \boldsymbol{x}_{\mathbf{2}}$ \\
\hline $\mathrm{b}_{1,1}=-0,02053$ & $\mathrm{~b}_{2,1}=0,01318$ \\
\hline $\mathrm{b}_{1,2}=-0,1656$ & $\mathrm{~b}_{2,2}=-0,004897$ \\
\hline $\mathrm{b}_{1,3}=0,0005185$ & $\mathrm{~b}_{2,3}=0,004672$ \\
\hline $\mathrm{b}_{1,4}=0,01107$ & $\mathrm{~b}_{2,4}=-0,005345$ \\
\hline $\mathrm{b}_{1,5}=0,0629$ & $\mathrm{~b}_{2,5}=-0,005947$ \\
\hline $\mathrm{b}_{1,6}=-0,0007888$ & $\mathrm{~b}_{2,6}=0,001264$ \\
\hline $\mathrm{b}_{1,7}=0,0006964$ & $\mathrm{~b}_{2,7}=-0,002227$ \\
\hline $\mathrm{b}_{1,8}=0,009514$ & $\mathrm{~b}_{2,8}=-0,001559$ \\
\hline $\mathrm{b}_{1,9}=0,02173$ & $\mathrm{~b}_{2,9}=0,0008845$ \\
\hline $\mathrm{b}_{1,10}=0,0002861$ & $\mathrm{~b}_{2,10}=-0,0008455$ \\
\hline $\mathrm{b}_{1,11}=-0,0002881$ & $\mathrm{~b}_{2,11}=-0,000375$ \\
\hline $\mathrm{b}_{1,12}=-0,004257$ & $\mathrm{~b}_{2,12}=0,001152$ \\
\hline $\mathrm{b}_{1,13}=-0.01013$ & $\mathrm{~b}_{2,13}=0.001337$ \\
\hline
\end{tabular}

Isolando os coeficientes de cada termo da equação polinomial, obtemos a equação

$$
y_{s s}\left(u_{1}, u_{2}\right)=\left[\begin{array}{llll}
b_{1} b_{2} b_{3} b_{4} b_{5} b_{6} b_{7} b_{8} b_{9} b_{10} b_{11} b_{12} b_{13}
\end{array}\right]\left[\begin{array}{c}
u_{1} \\
u_{2} \\
u_{1}^{2} \\
u_{1} u_{2} \\
u_{2}^{2} \\
u_{1}^{3} \\
u_{1}^{2} u_{2} \\
u_{1} u_{2}^{2} \\
u_{2}^{3} \\
u_{1}^{3} u_{2} \\
u_{2}^{2} u_{2}^{2} \\
u_{1} u_{2}^{3} \\
u_{2}^{4}
\end{array}\right],
$$

em que $y_{s s}$ é a variável de saída estacionária. A variável de entrada modificada ajustada $\phi_{\text {mod }}$ é então

$$
\phi_{\text {mod }}=\left[\begin{array}{llllllllllllllll}
u_{1} & u_{2} & u_{1}^{2} & u_{1} u_{2} & u_{2}^{2} & u_{1}^{3} & u_{1}^{2} u_{2} & u_{1} u_{2}^{2} & u_{2}^{3} & u_{1}^{3} 3 u_{2} & u_{2}^{2} u_{2}^{2} & u_{1} u_{2}^{3} & u_{2}^{4}
\end{array}\right]^{T} .
$$


Observa-se pelas Figuras 18 e 19 que os modelos obtidos tiveram um bom ajuste, portanto, sendo capaz de representar bem o comportamento do sistema. A Tabela 9 apresenta algumas métricas para avaliar a qualidade do ajuste.

Tabela 9: Qualidade do Ajuste.

\begin{tabular}{lcc}
\hline Parâmetros & $u_{1} \times u_{2} \times x_{1}$ & $u_{1} \times u_{2} \times x_{2}$ \\
\hline SSE & $1,494 \cdot 10^{-1}$ & $2,275 \cdot 10^{-3}$ \\
$\mathbf{R}^{2}$ & 0,9944 & 0,9946 \\
\hline
\end{tabular}

\subsubsection{2.}

Modelo dinâmico linear em espaço de estado

Para obter o modelo modificado em espaço de estado conforme Equação

$$
\begin{aligned}
& \frac{\partial x}{\partial t}=A x+B_{\text {mod }} \phi_{\text {mod }} \\
& y=C x,
\end{aligned}
$$

é substituído o termo $\phi_{\text {mod }}$ obtido na sessão anterior e a matriz $B_{\text {mod }}$, em que esta foi reformulada para incorporar os coeficientes das funções polinomiais. Logo, o modelo modificado pode ser representado como

$$
\frac{\partial x}{\partial t}=\left[\begin{array}{cc}
-\frac{1}{\tau_{1}} & 0 \\
0 & -\frac{1}{\tau_{2}}
\end{array}\right] x+\left[\begin{array}{ccc}
b_{1,1} \frac{k p_{1,1}}{\tau_{1}} & \ldots & b_{1,13} \frac{k p_{1,13}}{\tau_{1}} \\
b_{2,1} \frac{k p_{2,1}}{\tau_{2}} & \ldots & b_{2,13} \frac{k p_{2,13}}{\tau_{2}}
\end{array}\right]\left[\begin{array}{c}
u_{1} \\
u_{2} \\
u_{1}^{2} \\
u_{1} u_{2} \\
u_{2}^{2} \\
u_{1}^{3} \\
u_{1}^{2} u_{2} \\
u_{1} u_{2}^{2} \\
u_{2}^{3} \\
u_{1}^{3} u_{2} \\
u_{2}^{2} u_{2}^{2} \\
u_{1} u_{2}^{3} \\
u_{2}^{4}
\end{array}\right]
$$

$y=\left[\begin{array}{ll}1 & 0 \\ 0 & 1\end{array}\right] x$ 


\subsubsection{3.}

\section{Identificação dos parâmetros do modelo}

A identificação do modelo em espaço de estado foi feita de forma a estimar os parâmetros $\tau_{1}, \tau_{2}, k p_{1,1}$ a $k p_{1,13}$ e $k p_{2,1}$ a $k p_{2,13}$, minimizando o SSE dados dinâmicos experimentais do sistema e o valor calculado, através da função fminsearch do Matlab. Os dados utilizados para a identificação foram para uma faixa de operação menor mais próxima da faixa operacional de projeto, devido a diferença muito grande de comportamento de resposta não-linear quanto mais distante o ponto de operação estiver do ponto de projeto, dificultando a modelagem do sistema.

Os valores passaram a ser de $\mathrm{R}$ entre 0,9 e 1,4 $\mathrm{kmol} / \mathrm{s}$ e de T entre $335 \mathrm{e}$ 340 K. A convergência pode ser observada na Figura 20, em que o SSE convergiu para o valor de 0,021 ao final da estimação.

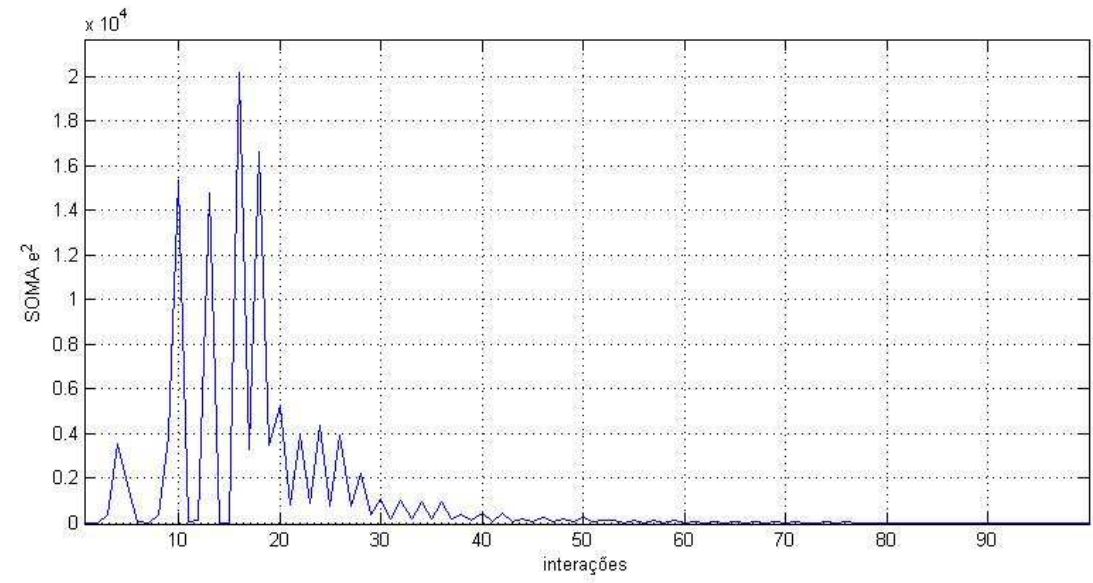

Figura 20: Convergência do SSE para um valor mínimo a cada interação da estimação dos parâmetros.

As Figuras 21 e 22 apresentam uma comparação entre os valores medidos e preditos de $x_{1}$ e $x_{2}$, respectivamente. O SSE entre o valor real e o valor predito para $x_{1}$ foi de 0,2787 e para $x_{2}$ foi de 0,3287 . 


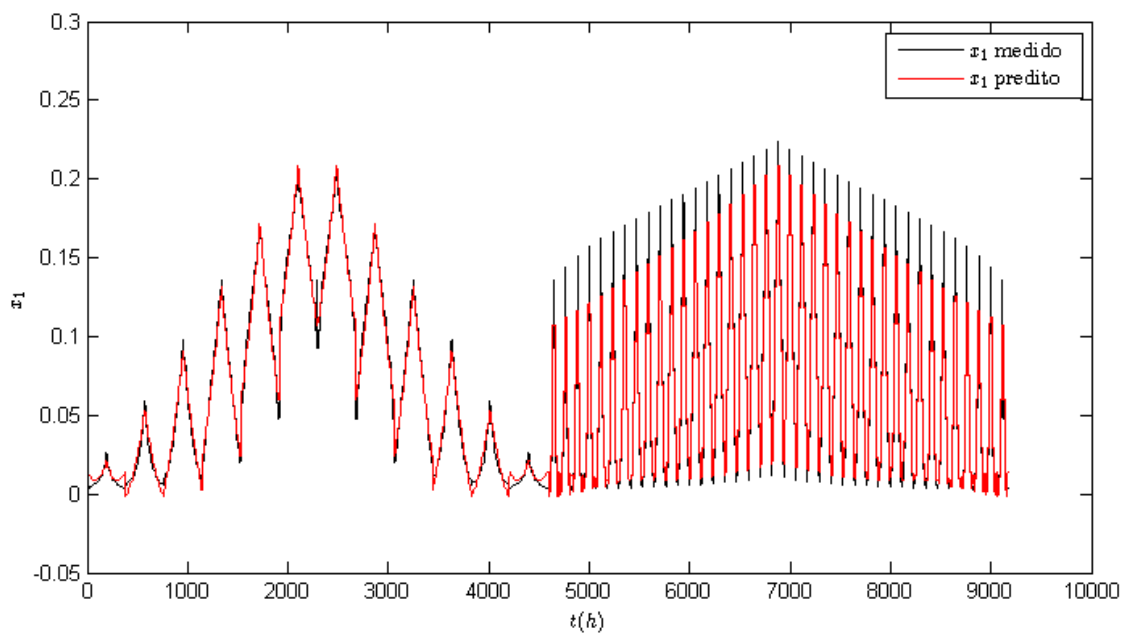

Figura 21: Gráfico de $x_{1}$ medido e predito.

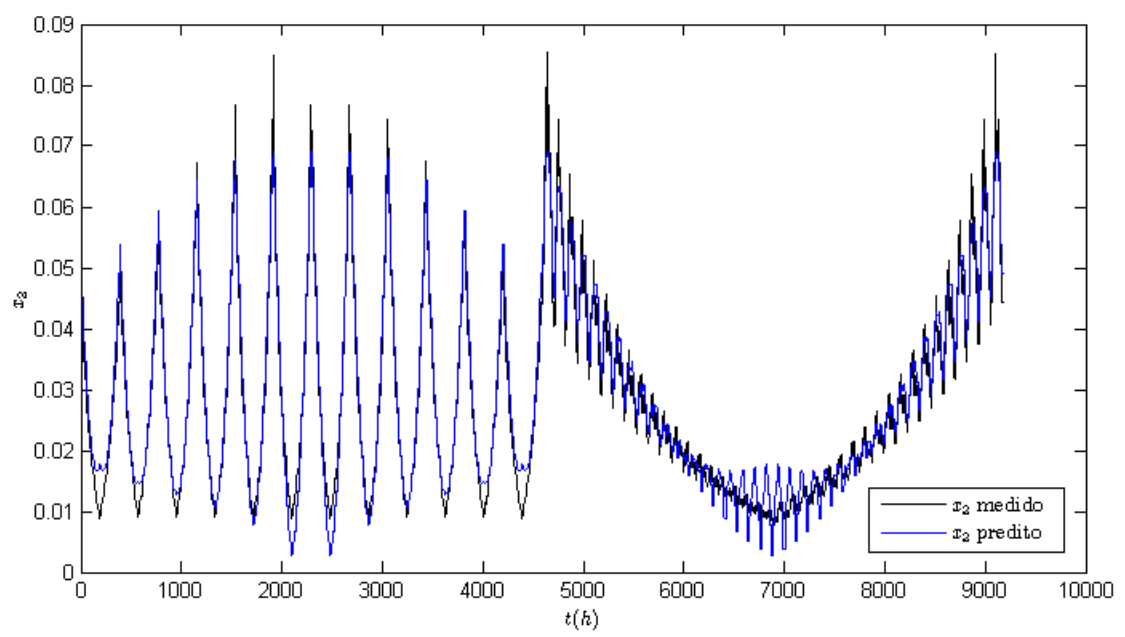

Figura 22: Gráfico de $x_{2}$ medido e predito.

Os parâmetros identificados estão apresentados na Tabela 10 . 
Tabela 10: Parâmetros estimados para o modelo linear.

\begin{tabular}{|c|c|c|}
\hline & $x_{1}$ & $x_{2}$ \\
\hline $\operatorname{tau}_{1}$ & $=0,3969$ & $\mathrm{tau}_{2}=0,1987$ \\
\hline $\mathrm{kp}_{1,1}$ & $=0,0014$ & $\mathrm{kp}_{2,1}=-0,0001$ \\
\hline $\mathrm{kp}_{1,2}$ & $=-0,0305$ & $\mathrm{kp}_{2,2}=-0,0001$ \\
\hline $\mathrm{kp}_{1,3}$ & $=0,0$ & $\mathrm{kp}_{2,3}=-0$, \\
\hline $\mathrm{kp}_{1,4}$ & $=-0,1410$ & $\mathrm{kp}_{2,4}=0,0000$ \\
\hline $\mathrm{kp}_{1,5}$ & $=-0,0025$ & $\mathrm{kp}_{2,5}=-0$, \\
\hline $\mathrm{kp}_{1,6}$ & $=-0,0004$ & $\mathrm{kp}_{2,6}=0,0009$ \\
\hline $\mathrm{kp}_{1,7}$ & $=0,1944$ & $\mathrm{kp}_{2,7}=0,0152$ \\
\hline $\mathrm{kp}_{1,8}$ & $=0,2205$ & $\mathrm{kp}_{2,8}=0,0001$ \\
\hline $\mathrm{kp}_{1,9}$ & $=0,0027$ & $\mathrm{kp}_{2,9}=-0,0$ \\
\hline $\mathrm{kp}_{1,10}$ & $=0,0124$ & $\mathrm{kp}_{2,10}=-0,0004$ \\
\hline $\mathrm{kp}_{1,11}$ & $=-0,0191$ & $\mathrm{kp}_{2,11}=-0,0025$ \\
\hline $\mathrm{kp}_{1,12}$ & $=-0,0725$ & $\mathrm{kp}_{2,12}=-0$, \\
\hline $\mathrm{kp}_{1,13}$ & $=-0,0011$ & $\mathrm{kp}_{2,13}=-0,0001$ \\
\hline
\end{tabular}

\subsubsection{4.}

\section{Estimador de Horizonte Móvel}

O estimador de horizonte móvel foi incluído de forma a atualizar o modelo interno do controlador preditivo com a estimação dos parâmetros $x_{1}=\mathrm{C} 3 \mathrm{~B}$ e $x_{2}=$ C4D, frente a ruídos de medição. Como as variáveis $x_{1}$ e $x_{2}$ são referentes a frações molares, seus valores são restritos à faixa de valores entre 0 e 1.

A estimação foi feita com os dados de simulação, de forma offline.

A Figura 23 mostra as estimativas das variáveis $x_{1}$ e $x_{2}$ juntamente com os respectivos valores obtidos experimentalmente sem ruído e com ruído aplicado de $7 \%$ a este. Observa-se boa estimativa com o modelo de Hammerstein, concluindo que este representa satisfatoriamente o comportamento real do sistema. 

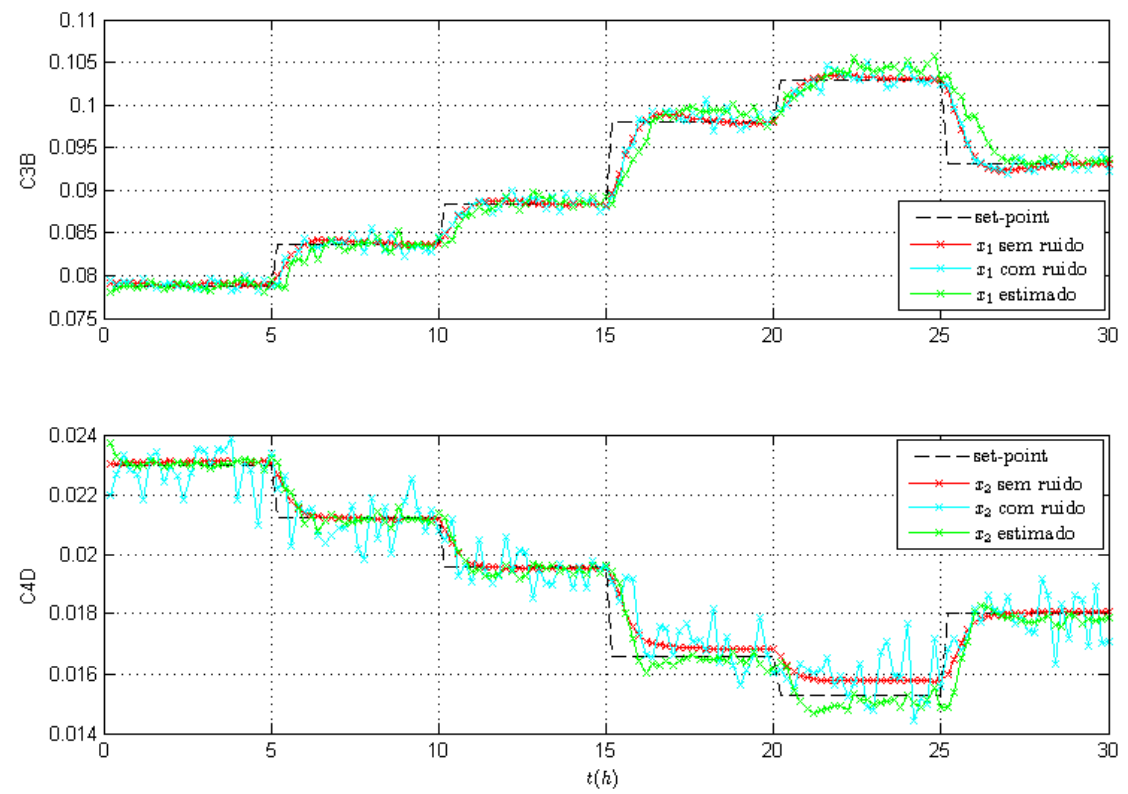

Figura 23: Gráfico do desempenho do estimador na presença de ruído de medição.

A Tabela 11 indica os parâmetros de sintonia do estimador.

Tabela 7: Parâmetros de sintonia do MHE.

\begin{tabular}{|c|c|}
\hline $\begin{array}{c}\text { Matriz de } \\
\text { covariância do ruído } \\
\text { do modelo }(Q)\end{array}$ & {$\left[\begin{array}{cc}10^{-5} & 0 \\
0 & 10^{-5}\end{array}\right]$} \\
\hline $\begin{array}{c}\text { Matriz de } \\
\text { covariância do ruído } \\
\text { de observação }(R)\end{array}$ & {$\left[\begin{array}{cc}0.07 & 0 \\
0 & 0.07\end{array}\right]$} \\
\hline $\begin{array}{c}\text { Matriz de } \\
\text { covariância do erro } \\
\text { de estimação inicial } \\
(\pi)\end{array}$ & {$\left[\begin{array}{cc}4 \cdot 10^{2} & 4,52 \cdot 10^{4} \\
4,52 \cdot 10^{4} & 10^{6}\end{array}\right]$} \\
\hline
\end{tabular}

\subsection{2}

\section{Modelo por Redes Neurais}

Os dados foram analisados para identificar os parâmetros da nãolinearidade dinâmica do modelo da coluna de destilação, de forma a encontrar os parâmetros da rede neural para cada uma das saídas. Assim, foram testadas 
redes de 1 a 10 neurônios na camada escondida para as variáveis de estado, conforme Tabela 12.

A Tabela 12 apresenta os resultados do ajuste da Rede Neural para os testes realizados de 1 a 10 neurônios na camada escondida.

Tabela 12: Qualidade do ajuste das redes.

\begin{tabular}{|c|c|c|c|c|c|c|c|c|c|}
\hline \multicolumn{5}{|c|}{ СзB } & \multicolumn{5}{|c|}{ C4D } \\
\hline \multirow{2}{*}{ Modelo } & \multirow{2}{*}{ Topologia } & \multicolumn{2}{|c|}{ Treinamento } & \multirow{2}{*}{$\begin{array}{c}\text { Teste } \\
\mathbf{R}\end{array}$} & \multirow{2}{*}{ Modelo } & \multirow{2}{*}{ Topologia } & \multicolumn{2}{|c|}{ Treinamento } & \multirow{2}{*}{$\begin{array}{c}\text { Teste } \\
\mathbf{R}\end{array}$} \\
\hline & & SSE & $\mathbf{R}$ & & & & SSE & $\mathbf{R}$ & \\
\hline 1 & $3-[1]-1$ & $3,63 \cdot 10^{-2}$ & 0,99987 & 0,99959 & 11 & $3-[1]-1$ & $9,02 \cdot 10^{-4}$ & 0,99995 & 0,99986 \\
\hline 2 & $3-[2]-1$ & $7,60 \cdot 10^{-3}$ & 0,99997 & 0,99993 & 12 & $3-[2]-1$ & $8,87 \cdot 10^{-4}$ & 0,99995 & 0,99986 \\
\hline 3 & $3-[3]-1$ & $7,20 \cdot 10^{-3}$ & 0,99997 & 0,99994 & 13 & $3-[3]-1$ & $5,39 \cdot 10^{-4}$ & 0,99997 & 0,99992 \\
\hline 4 & $3-[4]-1$ & $1,80 \cdot 10^{-3}$ & 0,99999 & 0,99998 & 14 & $3-[4]-1$ & $4,36 \cdot 10^{-4}$ & 0,99998 & 0,99993 \\
\hline 5 & $3-[5]-1$ & $5,96 \cdot 10^{-4}$ & 1 & 0,99999 & 15 & $3-[5]-1$ & $4,94 \cdot 10^{-4}$ & 0,99997 & 0,99992 \\
\hline 6 & $3-[6]-1$ & $4,13 \cdot 10^{-4}$ & 1 & 0,99999 & 16 & $3-[6]-1$ & $3,06 \cdot 10^{-4}$ & 0,99998 & 0,99993 \\
\hline 7 & $3-[7]-1$ & $2,82 \cdot 10^{-4}$ & 1 & 1 & 17 & $3-[7]-1$ & $3,11 \cdot 10^{-4}$ & 0,99998 & 0,99994 \\
\hline 8 & $3-[8]-1$ & $2,09 \cdot 10^{-4}$ & 1 & 1 & 18 & $3-[8]-1$ & $3,11 \cdot 10^{-4}$ & 0,99998 & 0,99993 \\
\hline 9 & 3-[9]-1 & $2,05 \cdot 10^{-4}$ & 1 & 1 & 19 & $3-[9]-1$ & $3,11 \cdot 10^{-4}$ & 0,99998 & 0,99994 \\
\hline 10 & $3-[10]-1$ & $2,16 \cdot 10^{-4}$ & 1 & 1 & 20 & $3-[10]-1$ & $3,03 \cdot 10^{-4}$ & 0,99998 & 0,99993 \\
\hline
\end{tabular}

Os modelos 4 e 14 da Tabela 12 representam as redes com 4 neurônios na camada escondida para ambas as saídas, com resultados satisfatórios. O modelo 4 apresenta treinamento da rede com índices de ajuste SSE $=1,80 \cdot 10^{-3} \mathrm{e}$ $R=0,99999$, e índice de ajuste de teste $R=0,99998$ para a saída C3B e o modelo 14 apresenta treinamento da rede com índices de ajuste SSE $=4,36 \cdot 10^{-4}$ e $R=$ 0,99998, e índice de ajuste de teste $\mathrm{R}=0,99993$ para a saída C4D.

O resultado do treinamento e do teste da rede de modelo 4 para $x_{1}$ é observado nas Figuras 24 e 25.

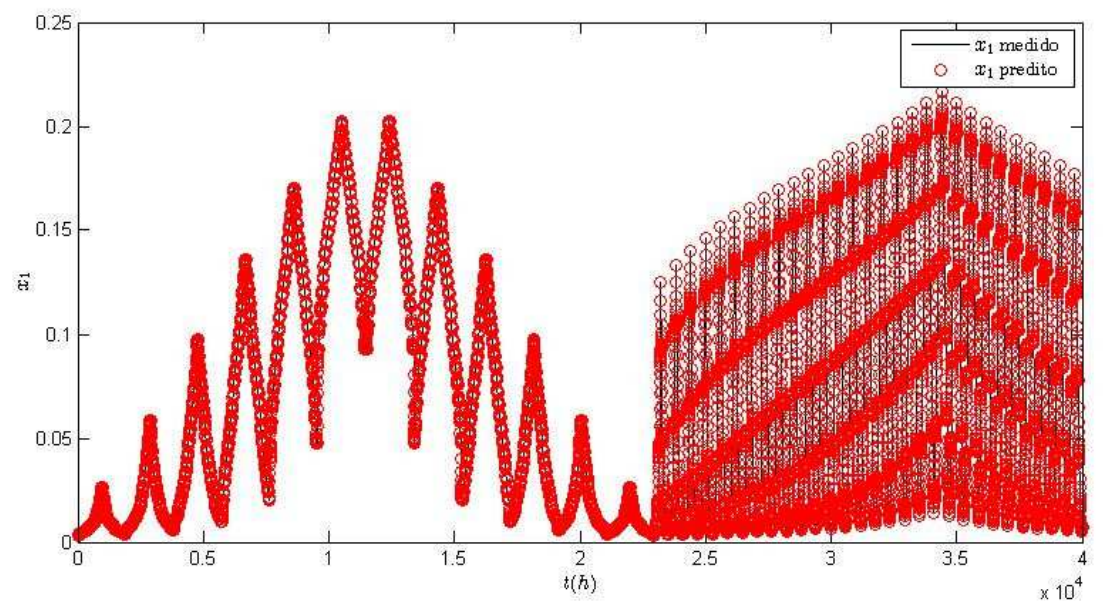

Figura 24: Treino da rede para $x_{1}$. 


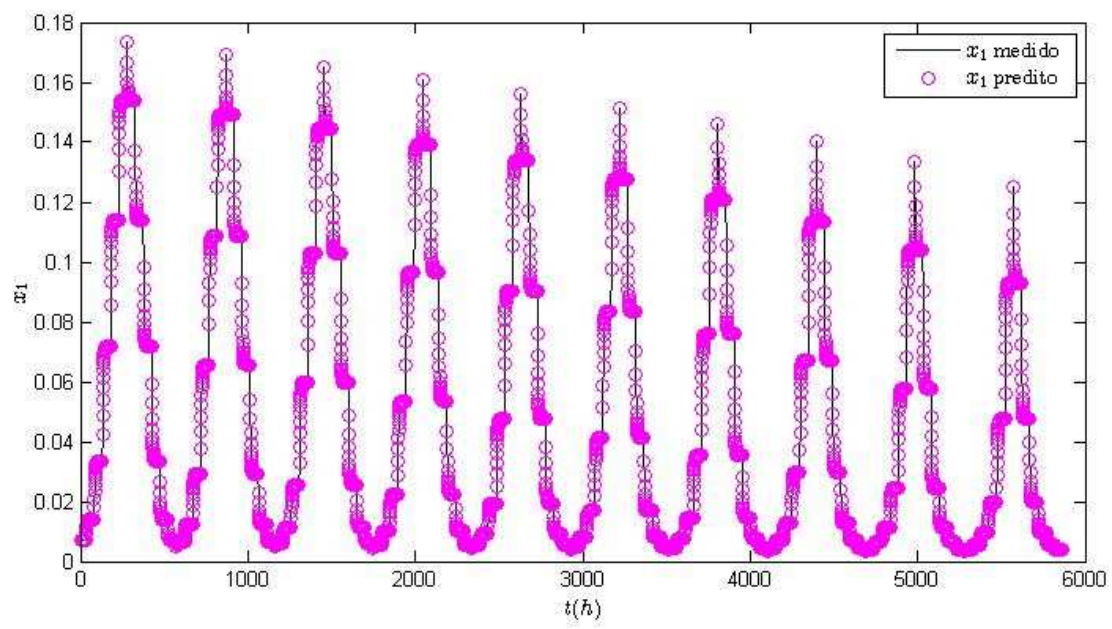

Figura 25: Teste da rede para $x_{1}$.

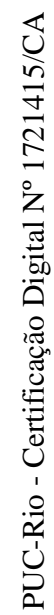

O resultado do treinamento e do teste da rede de modelo 14 para $x_{2}$ é observado nas Figuras 26 e 27.

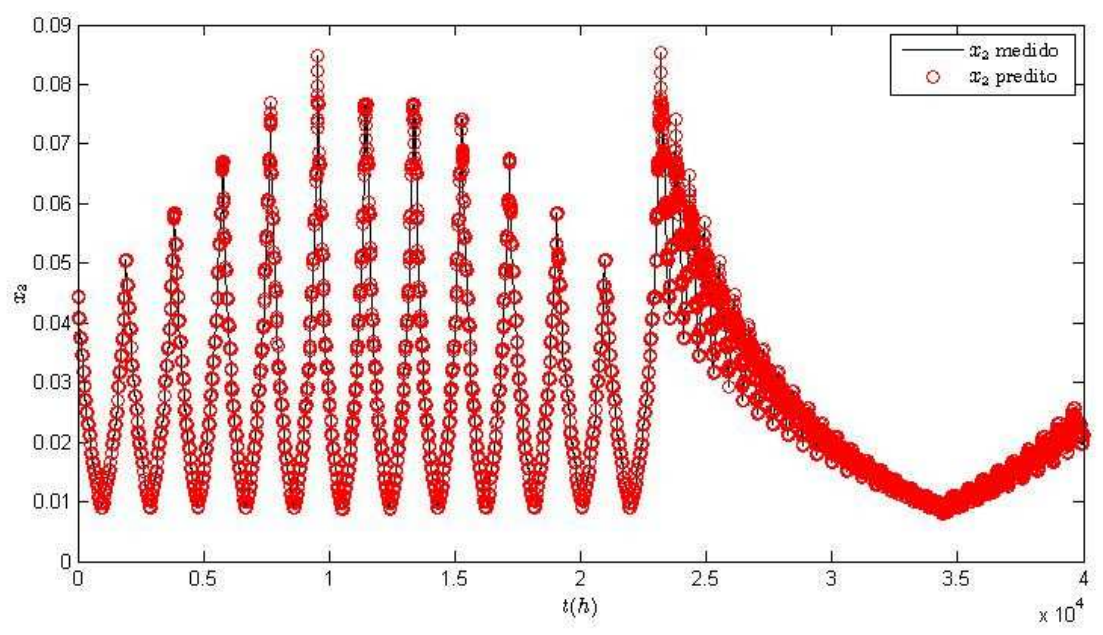

Figura 26: Treino da rede para $x_{2}$. 


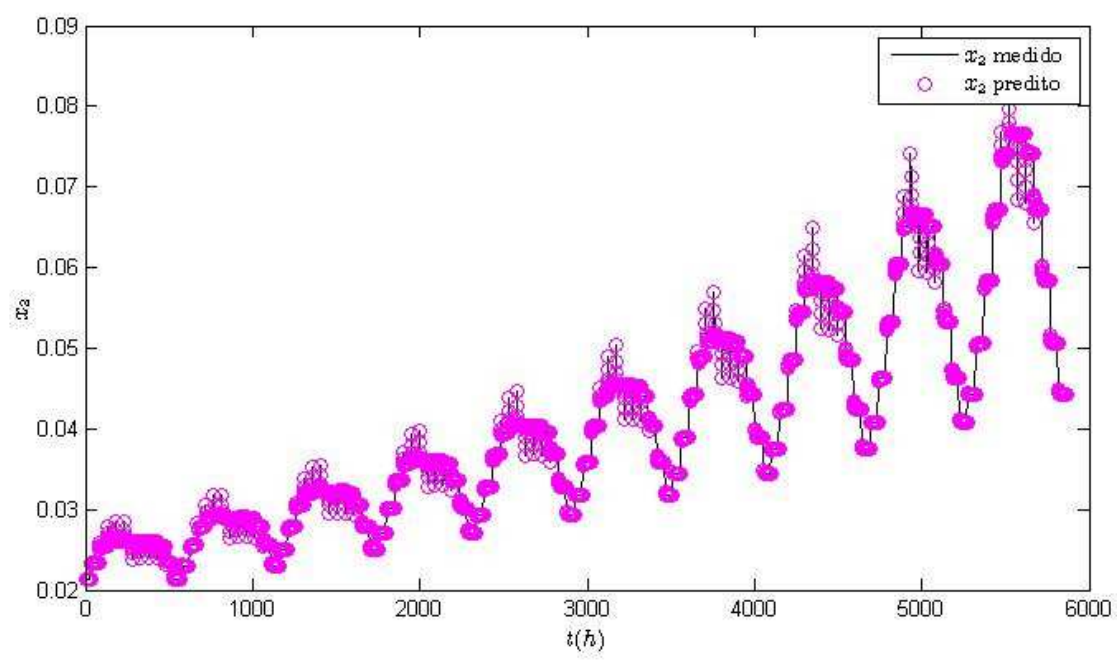

Figura 27: Teste da rede para $x_{2}$.

\subsubsection{1.}

\section{Modelo por Redes Neurais iterativo}

Como visto na seção 2.4.4.2, a estrutura do NN-NMPC é usada para prever saídas futuras passos a frente num horizonte de predicao $N_{p}$. A saída da primeira predicao $x(k+1)$ é usada como entrada do modelo para a próxima predição $x(k+$ 2) e assim por diante, até $x\left(k+N_{p}-1\right)$. Com esse método iterativo, múltiplas saídas podem ser preditas $N_{p}$ passos a frente. Assim, foi aplicado o método iterativo para os modelos 4 e 14 para prever $N_{p}=40$ passos a frente após um degrau, observadas nas Figuras 28 e 29 respectivamente.

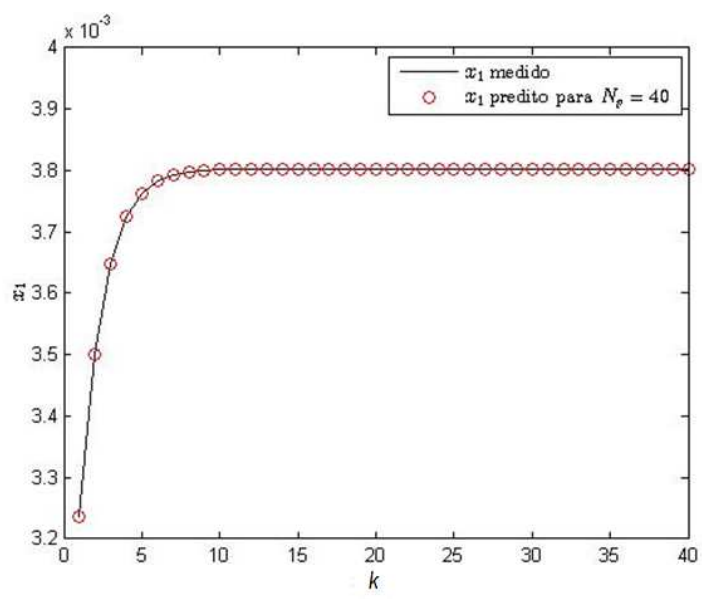

Figura 28: Teste da rede neural iterativa para $x_{1}$ e $N_{p}=40$. 


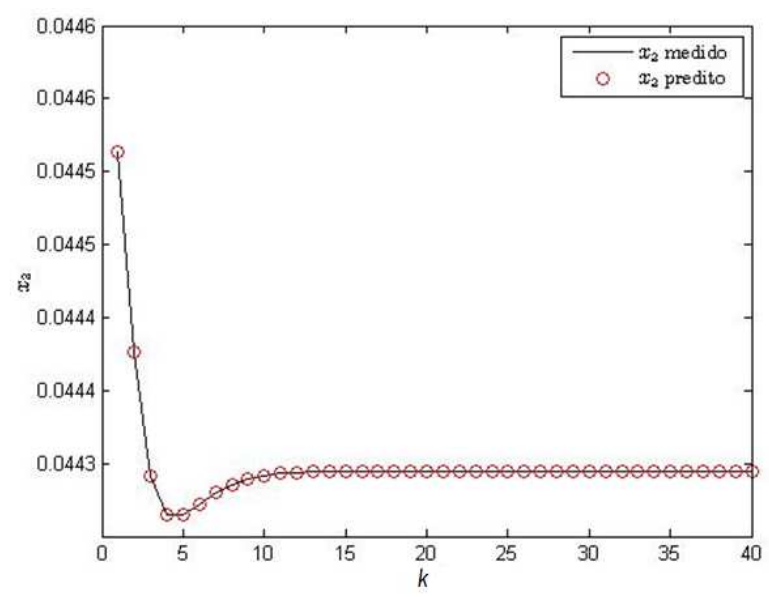

Figura 29: Teste da rede neural iterativa para $x_{2}$ e $N_{p}=40$.

\section{3.}

\section{Sintonia dos controladores}

Esta seção apresenta os resultados das sintonias dos controladores PID e NMPC.

\subsection{1.}

\section{Sintonia do controlador PID}

A sintonia dos controladores foi feita conforme descrição de Luyben (2006). Os parâmetros do controlador após a sintonia estão apresentados na Tabela 13.

Tabela 13: Sintonia dos controladores PI.

\begin{tabular}{|c|c|c|c|c|c|c|c|c|}
\hline Controle & PV & OP & ação & $\mathbf{k}_{\mathbf{p}}(\% / \%)$ & $\boldsymbol{\tau}_{\boldsymbol{i}}(\mathbf{m i n})$ & $\mathbf{P V}$ mín & PV máx & $\mathbf{S P}$ \\
\hline FIC-1 & Vazão F & $\mathrm{V}_{1}$ & reversa & 0,5 & 0,3 & 0 & 2 & $1 \mathrm{kmol} / \mathrm{s}$ \\
\hline FIC-2 & Vazão R & $\mathrm{V}_{\mathrm{R}}$ & reversa & 0,5 & 0,3 & 0 & 2,723 & $1 \mathrm{kmol} / \mathrm{s}$ \\
\hline PIC-1 & P prato 1 & $\mathrm{Q}_{\text {cond }}$ & reversa & 20 & 12 & 0 & 34,05 & $17,02 \mathrm{bar}$ \\
\hline LIC-1 & L prato1 & $\mathrm{V}_{12}$ & direta & 2 & 9999 & 0 & 10,2 & $5,1 \mathrm{~m}$ \\
\hline LIC-2 & L prato 32 & $\mathrm{V}_{11}$ & direta & 2 & 9999 & 0 & 12,7 & $6,35 \mathrm{~m}$ \\
\hline TIC-9 & T prato 9 & Qref & reversa & 1,15 & 14,41 & 320 & 370 & $337,4 \mathrm{~K}$ \\
\hline CC3B & C3B & TIC-9 & direta & 2.1 & 72 & 0 & 0.05 & 0.01 \\
\hline CC4D & C4D & FIC-2 & reversa & 1.5 & 68 & 0 & 0.05 & 0.03 \\
\hline
\end{tabular}




\subsection{2.}

\section{Sintonia dos controladores NMPC}

A sintonia do controle preditivo foi feita a partir das recomendações de sintonia feitas por Seborg et al. (2011), distutidas na Seção 2.3.4.

$\mathrm{Na}$ Figura 30, observa-se que o tempo de resposta $t_{s}$ para um degrau é diferente para cada variável, sendo de aproximadamente 3h para C3B e 1h para C4D.
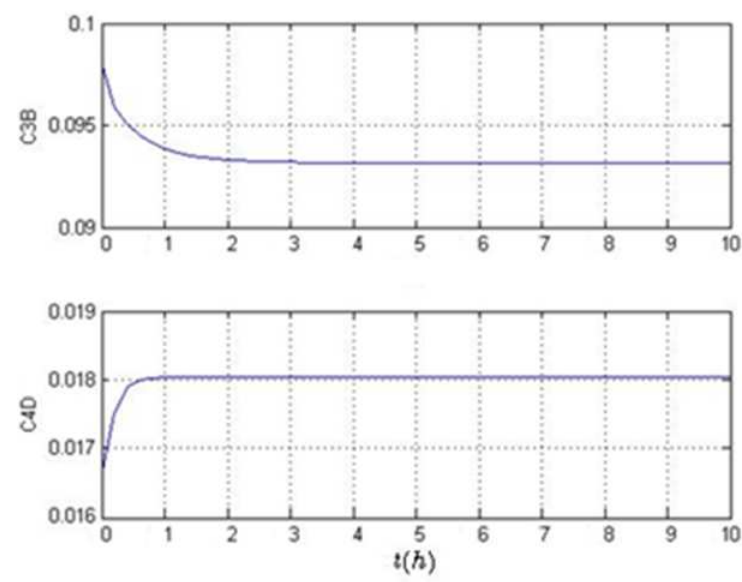

Figura 30: Tempo de resposta para as duas variáveis controladas.

O período de amostragem é de $\Delta t=0,1 \mathrm{~h}$, em que cada degrau tem um tempo de $10 \mathrm{~h}$ para a estabilização do sistema. Considerando $t_{s}=3 h$, então $N_{m}=$ $\frac{3}{0,1}=30$ e o valor estipulado está dentro da faixa recomendada.

Para horizonte de controle, teríamos então as faixas sugeridas $\frac{30}{3} \leq N_{c} \leq$ $\frac{30}{2}$, ou $5 \leq N_{c} \leq 20$, podendo ser um outro valor escolhido, sendo desejável valores mais baixos para $N_{c}$, devido ao esforço computacional e à agressividade do controle.

O horizonte de predição $N_{p}$ foi selecionado para ser $N_{p}=N_{c}+N_{m}$, conforme a recomendação. 


\subsubsection{1.}

\section{Sintonia do H-NMPC}

\section{a) Sintonia no horizonte de controle $N_{c}$}

Os primeiros testes de sintonia a serem feitos foram para o horizonte de controle $N_{c}$ e para o horizonte de predição $N_{p}$, de forma a percorrer vários valores de $N_{c}$, em que $N_{p}=N_{c}+30$. Foram testados, então, $N_{c}=\{4,5,6,7,9\}$. As demais sintonias foram fixadas em $Q_{y}=\left[10^{2} 0 ; 010^{2}\right]$ e $Q_{\Delta u}=\left[\left(10^{-4}\right)^{2} 0 ; 0\left(10^{-4}\right)^{2}\right]$.

As Figuras 31 e 32 apresentam o comportamento das saídas C3B e C4D, respectivamente, durante mudanças em suas trajetórias de referência. As Figuras 33 e 34 representam a variação das entradas $T$ e $R$, respectivamente, de forma a manter as variáveis controladas na trajetória de referência desejada.

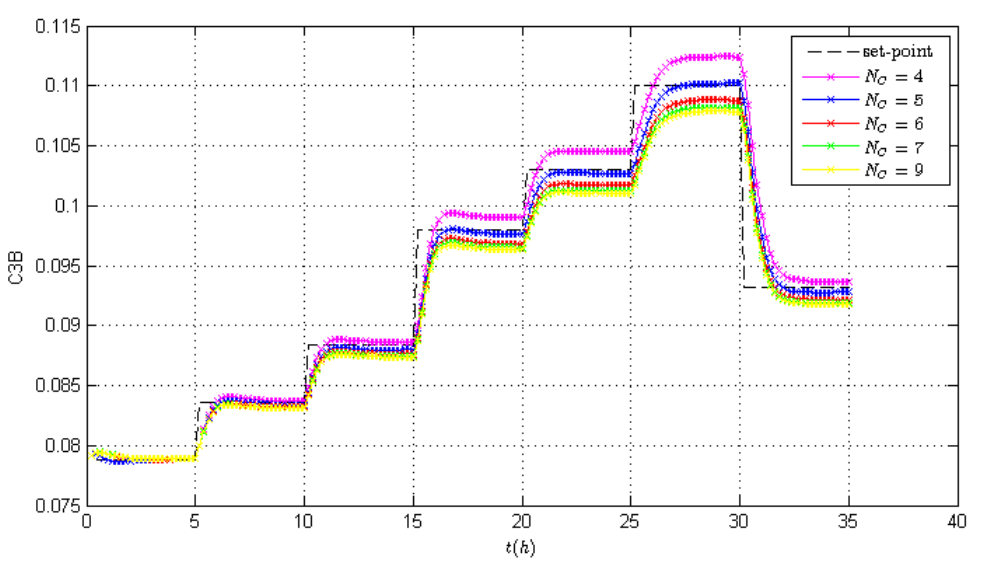

Figura 31: Sintonia do $N_{c}$ para saída C3B.

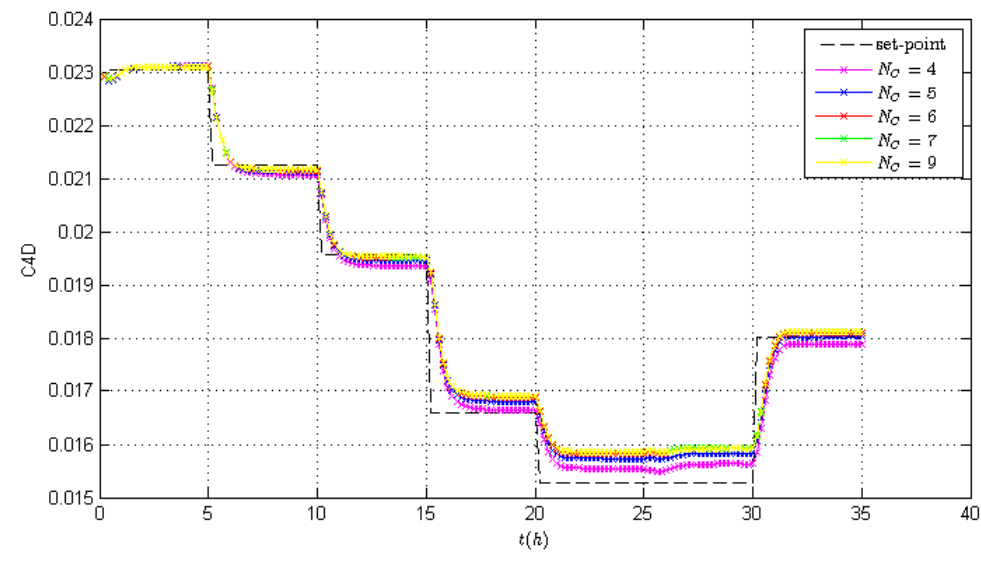

Figura 32: Sintonia do $N_{c}$ para saída C4D. 


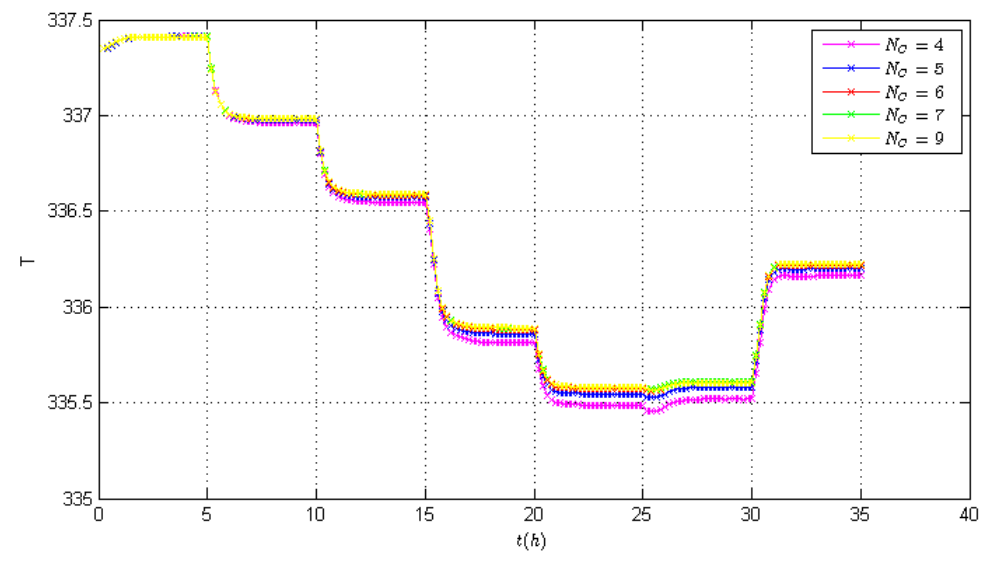

Figura 33: Sintonia do $N_{c}$ para entrada T.

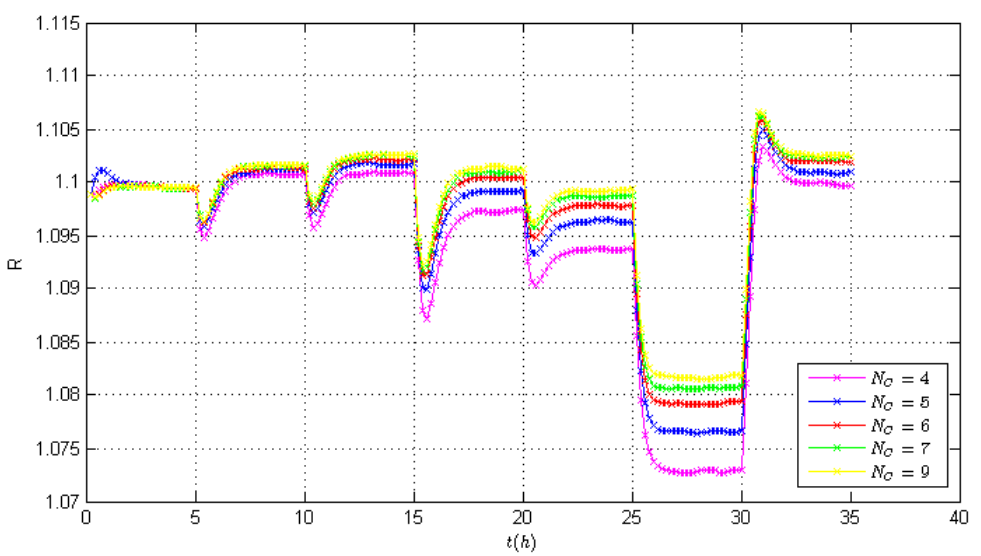

Figura 34: Sintonia do $N_{c}$ para entrada R.

Através dos gráficos gerados, o $N_{c}=5$ foi o valor que demonstrou melhor controle das trajetórias de referências. Foi observado também que o controle da resposta para a variável C3B possui maior peso do que para variável C4D.

Vale ressaltar que, quanto menor é o valor definido para $N_{c}$, mais agressivo se torna o controle.

\section{b) Sintonia $Q_{y}$}

Foi observado na sintonia anterior que a saída C3B ultrapassava seu valor de referência enquanto a saída C4D não o alcançava para alguns valores sintonizados. Então, foi feita aqui a sintonia de $Q_{y}$ para ponderar as saídas de acordo com sua importância relativa, em que $y_{1}=C 3 B$ e $y_{2}=C 4 D$. As demais 
sintonias foram fixadas em $N_{c}=5$ e $Q_{\Delta u}=\left[\left(10^{-4}\right)^{2} \quad 0 ; 0\left(10^{-4}\right)^{2}\right]$. Foram testados valores $Q_{y}=\left\{\left[\begin{array}{lll}1^{2} & 0 ; 0 & 10^{2}\end{array}\right],\left[\begin{array}{lll}10^{2} & 0 ; 0 & 1^{2}\end{array}\right],\left[\begin{array}{llll}10^{2} & 0 ; 0 & 10^{2}\end{array}\right],\left[\begin{array}{lll}2^{2} & 0 ; 0 & 10^{2}\end{array}\right],\left[\begin{array}{llll}5^{2} & 0 ; 0 & 10^{2}\end{array}\right]\right.$, $\left.\left[\begin{array}{llll}1^{2} & 0 ; 0 & 20^{2}\end{array}\right]\right\}$.

As Figuras 35 e 36 apresentam o comportamento das saídas C3B e C4D, respectivamente e as Figuras 37 e 38 representam a variação das entradas T e $\mathrm{R}$.

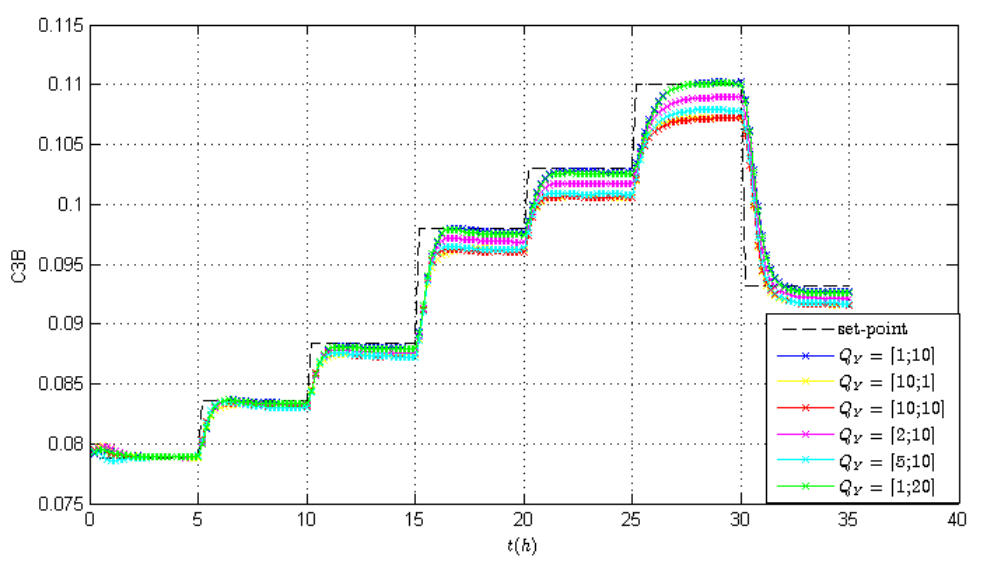

Figura 35: Sintonia do $Q_{y}$ para saída C3B.

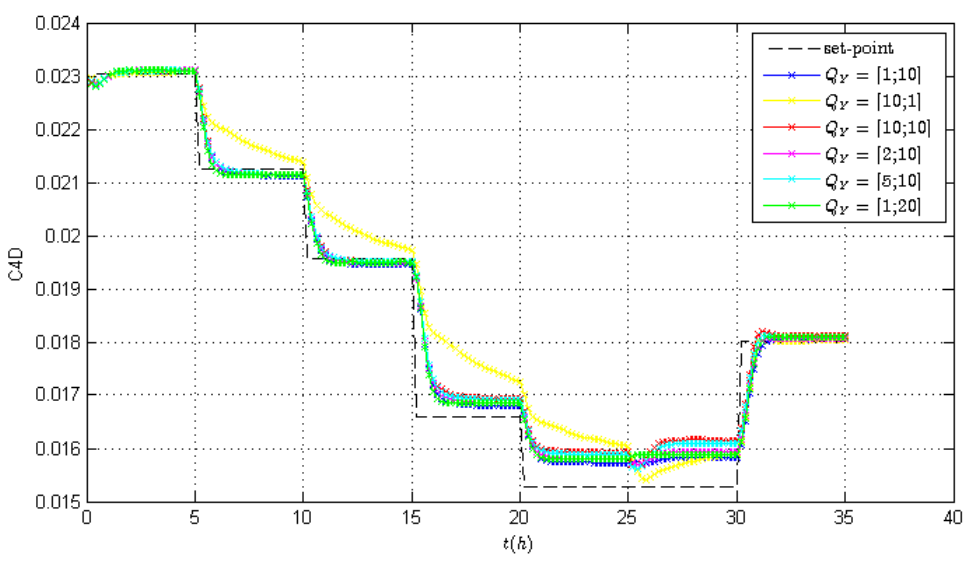

Figura 36: Sintonia do $Q_{y}$ para saída C4D. 


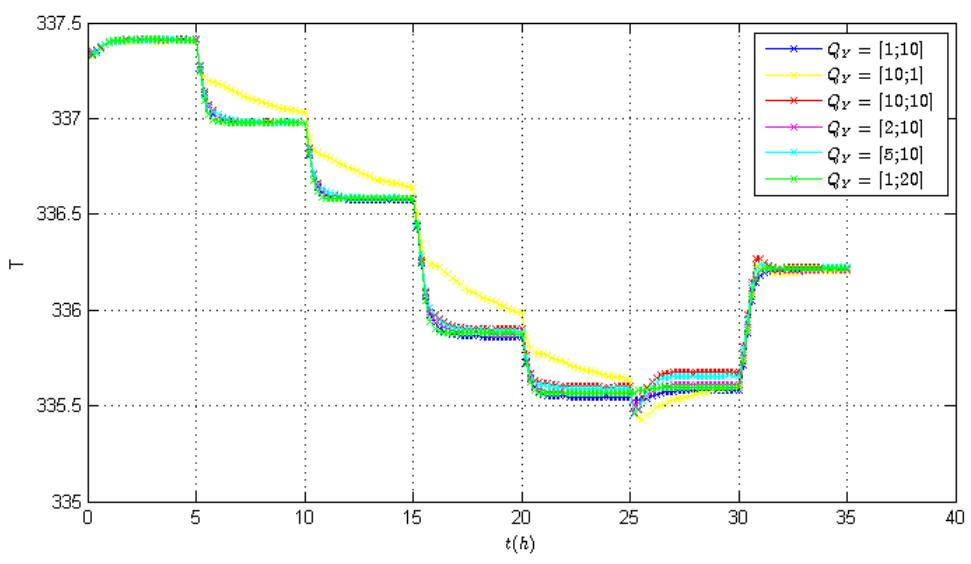

Figura 37: Sintonia do $Q_{y}$ para entrada T.

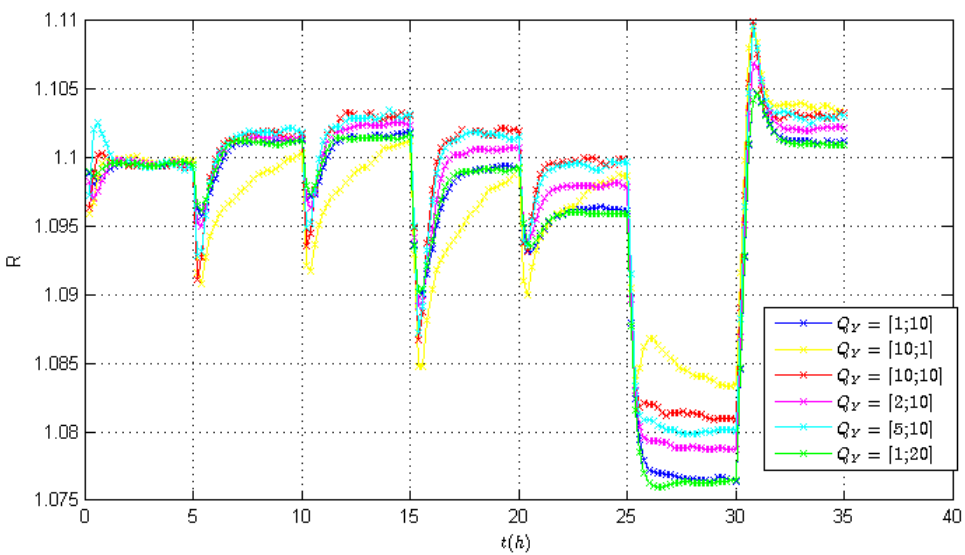

Figura 38: Sintonia do $Q_{y}$ para entrada R.

O valor para o melhor controle das respostas foi de $Q_{y}=\left[1^{2} 0 ; 010^{2}\right]$. Como esperado, foi necessário um peso maior para a saída C4D para uma melhor sintonia.

\section{c) Sintonia $Q_{\Delta u}$}

De forma similar a $Q_{y}, Q_{\Delta u}$ pondera as entradas $\mathrm{T}$ e $\mathrm{R}$ de acordo com sua importância relativa, em que $u_{1}=T$ e $u_{2}=R$. Como $Q_{\Delta u}$ está normalizado no algoritmo do modelo de Hammerstein, este foi considerado com mesmos valores pra ambas as entradas. As demais sintonias foram fixadas em $N_{c}=5$ e $Q_{y}=$ $\left[1^{2} 0 ; 010^{2}\right]$. Foram testados valores para $Q_{\Delta u}=$ $\left\{\left[\left(10^{-1}\right)^{2} 0 ; 0\left(10^{-1}\right)^{2}\right],\left[\left(10^{-2}\right)^{2} 0 ; 0\left(10^{-2}\right)^{2}\right],\left[\left(10^{-4}\right)^{2} 0 ; 0\left(10^{-4}\right)^{2}\right],\left[\left(10^{-6}\right)^{2} 0\right.\right.$; $\left.\left.0\left(10^{-6}\right)^{2}\right]\right\}$. 
As Figuras 39 e 40 apresentam o comportamento das saídas C3B e C4D, respectivamente e as Figuras 41 e 42 representam a variação das entradas T e $\mathrm{R}$.

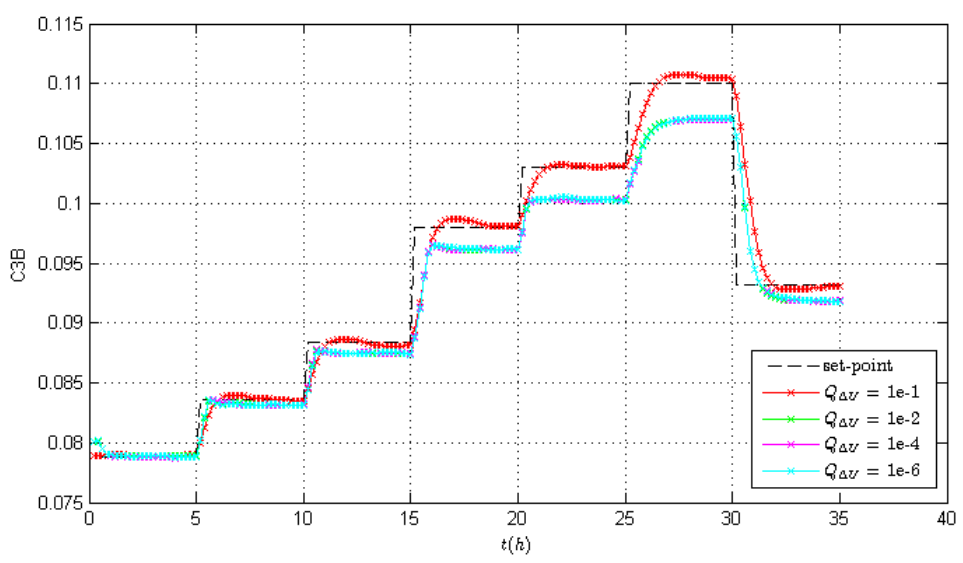

Figura 3913: Sintonia do $Q_{\Delta u}$ para saída C3B.

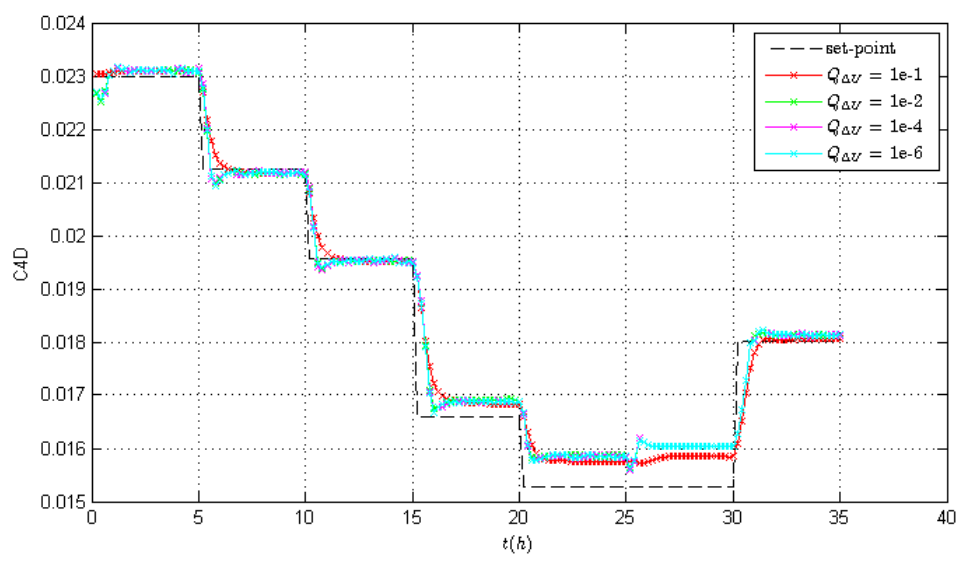

Figura 40: Sintonia do $Q_{\Delta u}$ para saída C4D. 


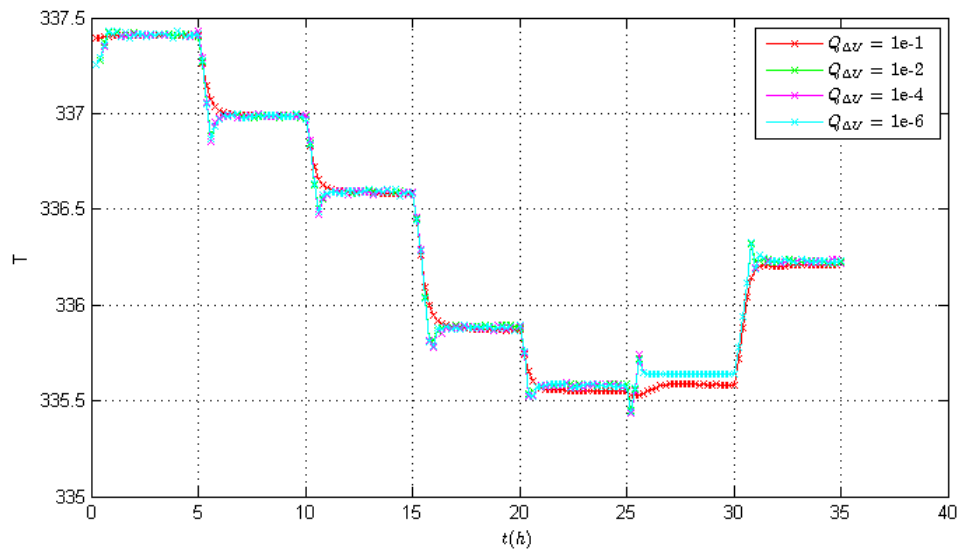

Figura 41: Sintonia do $Q_{\Delta u}$ para entrada T.

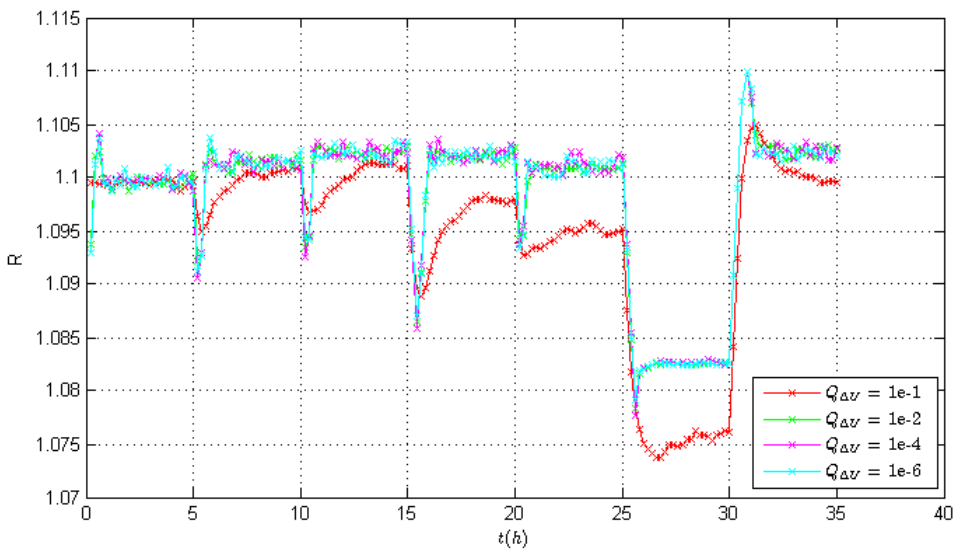

Figura 42: Sintonia do $Q_{\Delta u}$ para entrada R.

O valor para 0 melhor controle das respostas foi de $Q_{\Delta u}=$ $\left[\left(10^{-1}\right)^{2} 0 ; 0\left(10^{-1}\right)^{2}\right]$. Nota-se que o controlador MPC tende a se tornar mais conservador ao reduzir as magnitudes dos movimentos das variáveis manipuladas.

\subsubsection{2.}

\section{Sintonia do NN-NMPC}

\section{a) Sintonia no horizonte de controle $N_{c}$}

De forma similar a sintonia feita para o modelo de Hammerstein, foram feitos testes para o horizonte de controle $N_{c}$ e para o horizonte de predição $N_{p}$, de forma a percorrer vários valores de $N_{c}$, em que $N_{p}=N_{c}+30$. Foram testados aqui 
os mesmos valores $N_{c}=\{3,4,5,6,7\}$. As demais sintonias foram fixadas em $Q_{y}=$ $\left[10^{2} 0 ; 010^{2}\right]$ e $Q_{\Delta u}=\left[\left(10^{-4}\right)^{2} 0 ; 0\left(10^{-4}\right)^{2}\right]$.

As Figuras 43 e 44 apresentam o comportamento das saídas C3B e C4D, respectivamente, durante mudanças em suas trajetórias de referência. As Figuras 45 e 46 representam a variação das entradas $T$ e $R$, respectivamente, de forma a manter as variáveis controladas na trajetória de referência desejada.

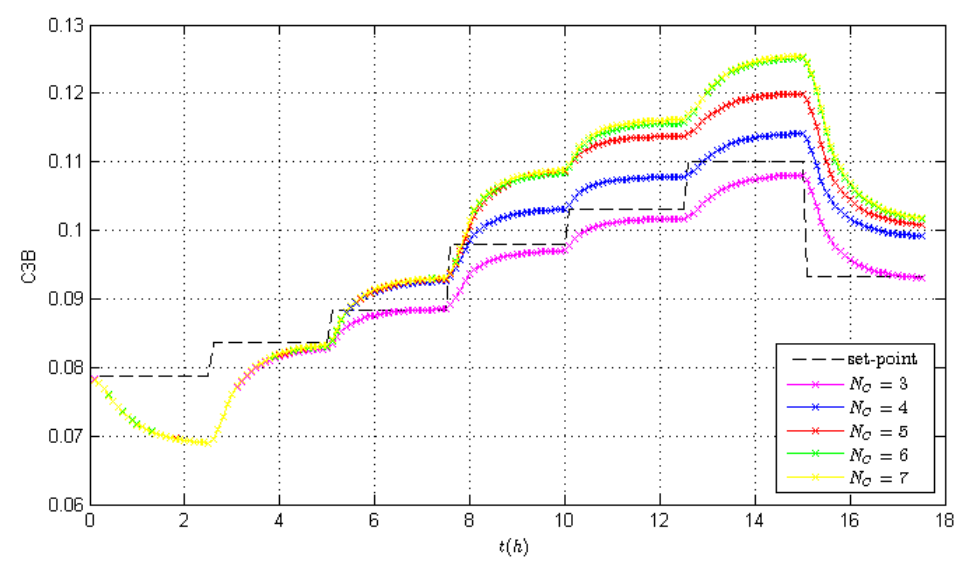

Figura 43: Sintonia do $N_{c}$ para saída C3B.

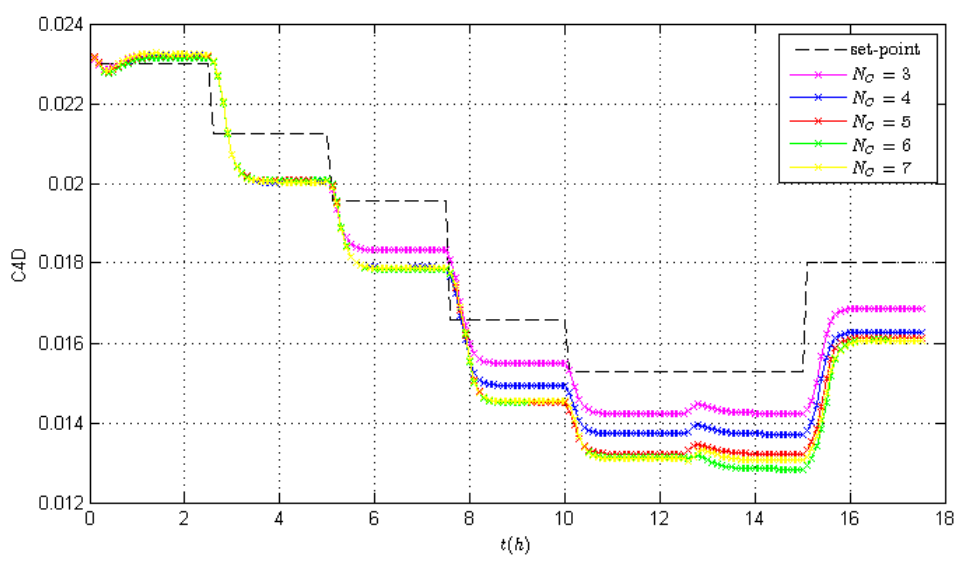

Figura 44: Sintonia do $N_{c}$ para saída C4D. 


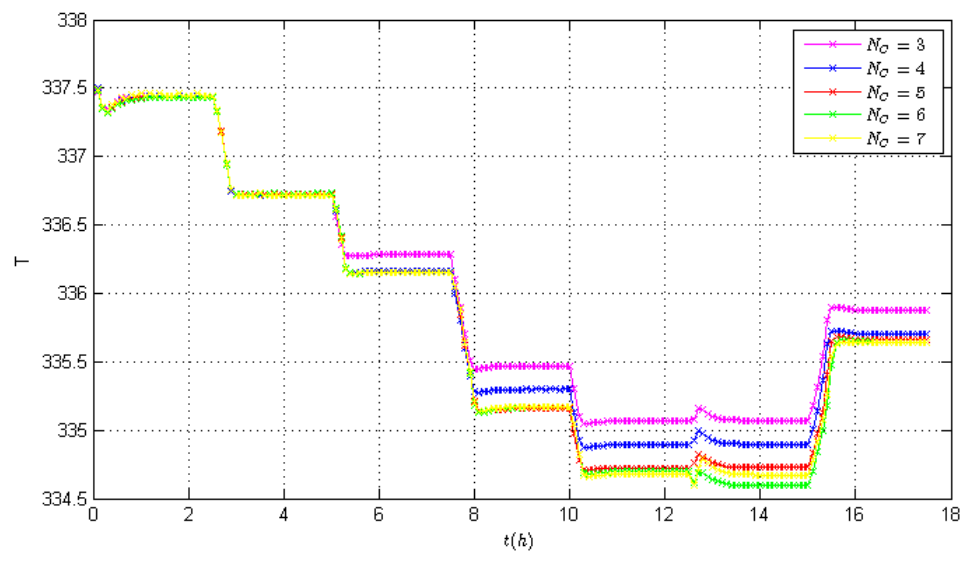

Figura 45: Sintonia do $N_{c}$ para entrada T.

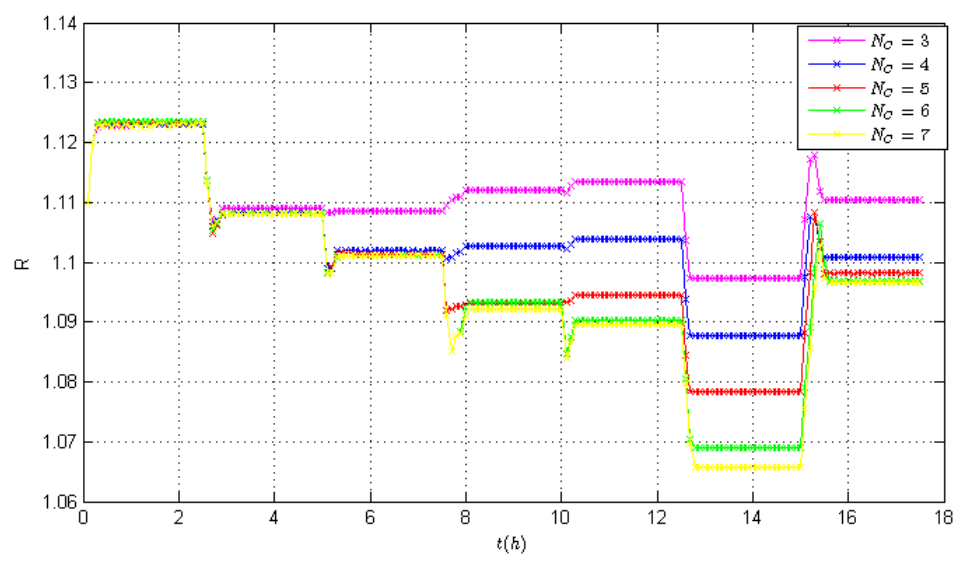

Figura 46: Sintonia do $N_{c}$ para entrada R.

Através dos gráficos gerados, o $N_{c}=3$ foi o valor que demonstrou melhor controle das trajetórias de referências e menor agressividade no controle.

\section{b) Sintonia $Q_{y}$}

Foi observado na sintonia anterior que ambas as saídas ultrapassavam cada vez mais seu valor de referência ao aumentar o valor de $N_{c}$. Foi feita aqui a sintonia de $Q_{y}$ para ponderar as saídas de acordo com sua importância relativa, em que as demais sintonias foram fixadas em $N_{c}=3$ e $Q_{\Delta u}=$

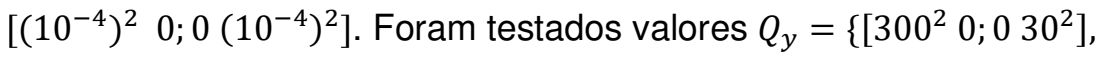

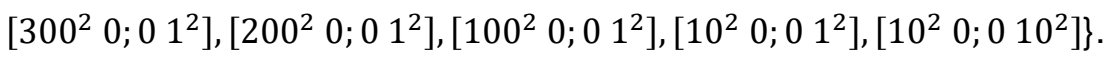


As Figuras 47 e 48 apresentam o comportamento das saídas C3B e C4D, respectivamente e as Figuras 49 e 50 representam a variação das entradas T e R.

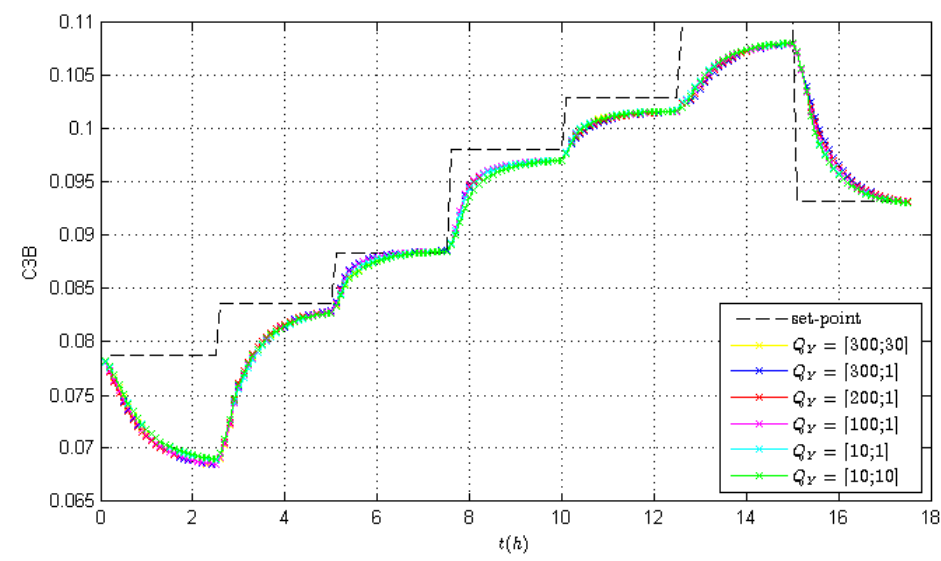

Figura 47: Sintonia do $Q_{y}$ para saída C3B.

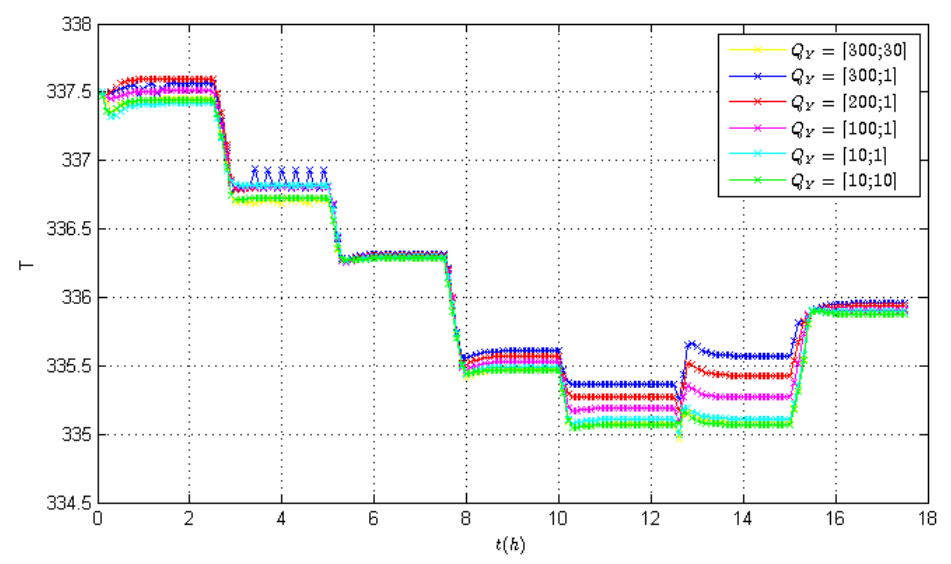

Figura 48: Sintonia do $Q_{y}$ para saída C4D. 


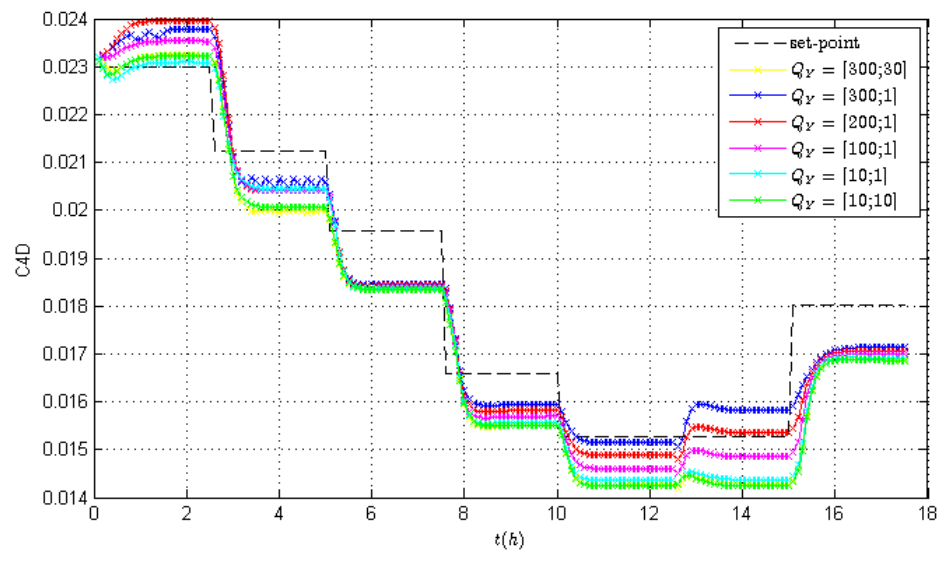

Figura 49: Sintonia do $Q_{y}$ para entrada T.

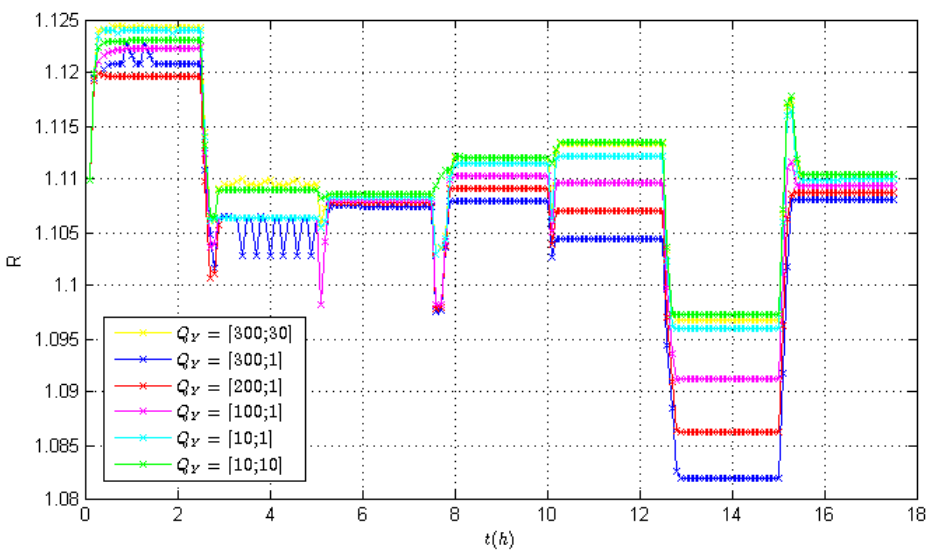

Figura 50: Sintonia do $Q_{y}$ para entrada R.

A variação do valor para $Q_{y}$ não pareceu afetar a saída C3B, mas teve bastante impacto na saída C4D, a qual teve resposta mais próxima à trajetória de referência com $Q_{y}=\left[200^{2} 0 ; 01^{2}\right]$.

\section{a) Sintonia $Q_{\Delta u}$}

De forma similar a $Q_{y}, Q_{\Delta u}$ pondera as entradas $\mathrm{T}$ e $\mathrm{R}$ de acordo com sua importância relativa. As demais sintonias foram fixadas em $N_{c}=3$ e $Q_{y}=$ $\left[300^{2} 0 ; 01^{2}\right]$. Foram testados valores para $Q_{\Delta u}=$ $\left\{\left[\left(10^{-4}\right)^{2} 0 ; 0\left(10^{-4}\right)^{2}\right],\left[\left(10^{-4}\right)^{2} 0 ; 0\left(10^{-2}\right)^{2}\right],\left[\left(10^{-2}\right)^{2} 0 ; 0\left(10^{-4}\right)^{2}\right],\left[\left(10^{-6}\right)^{2} 0\right.\right.$; $\left.\left.0\left(10^{-4}\right)^{2}\right],\left[\left(10^{-3}\right)^{2} 0 ; 0\left(10^{-1}\right)^{2}\right]\right\}$. 
As Figuras 51 e 52 apresentam o comportamento das saídas C3B e C4D, respectivamente e as Figuras 53 e 54 representam a variação das entradas T e $\mathrm{R}$.

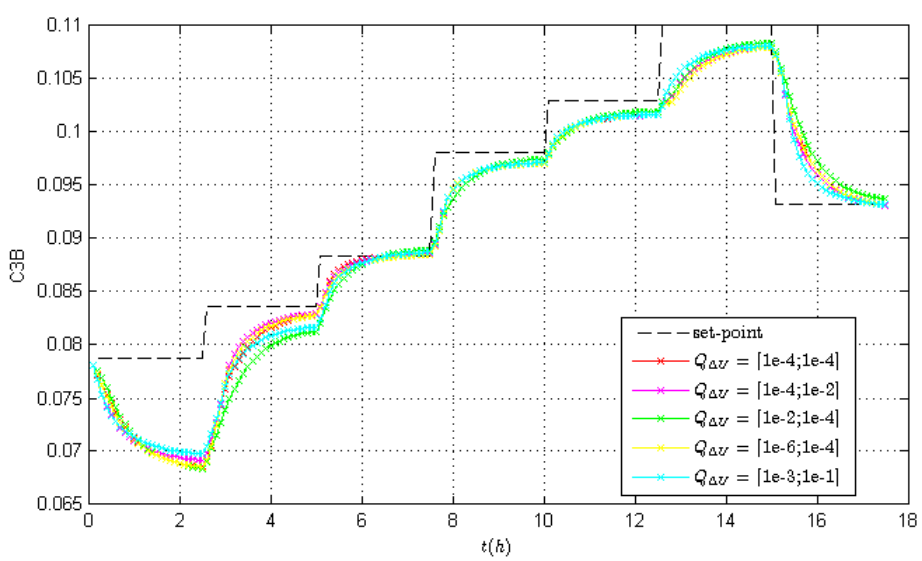

Figura 51: Sintonia do $Q_{\Delta u}$ para saída C3B.

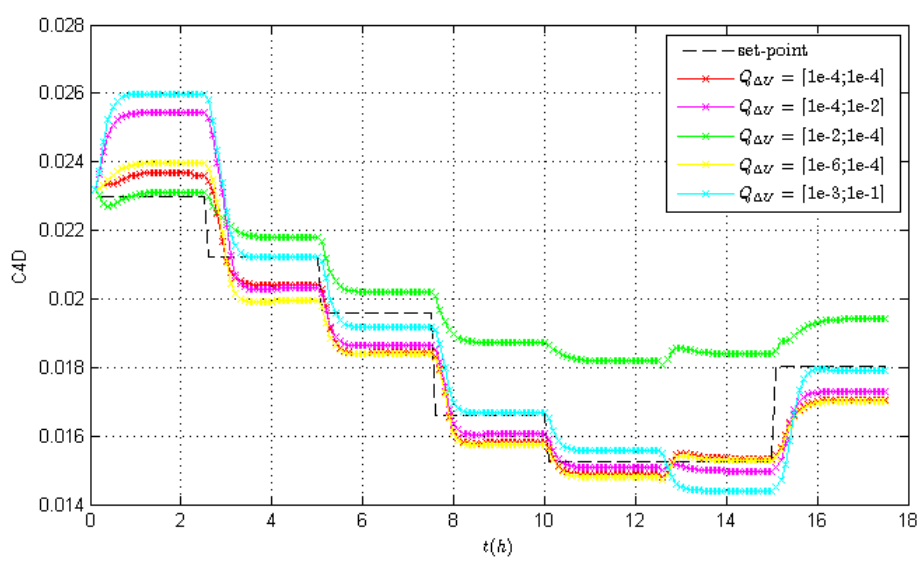

Figura 52: Sintonia do $Q_{\Delta u}$ para saída C4D. 


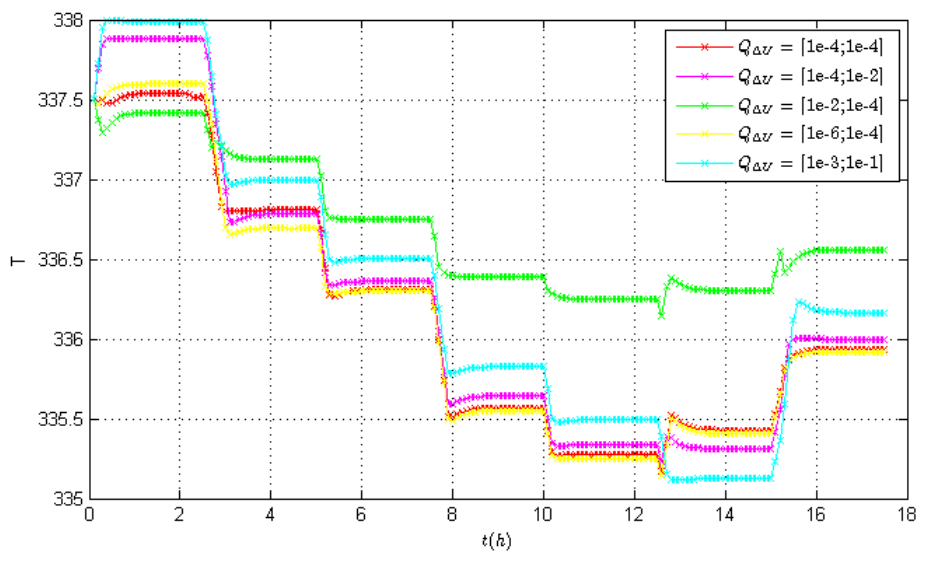

Figura 53: Sintonia do $Q_{\Delta u}$ para entrada T.

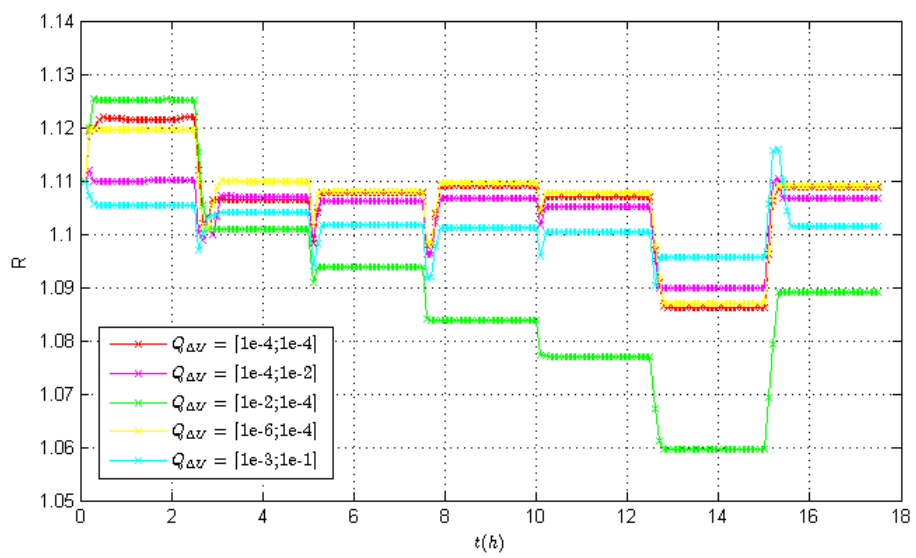

Figura 54: Sintonia do $Q_{\Delta u}$ para entrada R.

De forma similar à sintonia do intem anterior, a variação do valor para $Q_{\Delta u}$ não pareceu afetar a saída C3B, mas teve bastante impacto na saída C4D. As respostas ficaram mais próximas à trajetória de referência com $Q_{\Delta u}=$ $\left[\left(10^{-4}\right)^{2} 0 ; 0\left(10^{-2}\right)^{2}\right]$, suprimindo mais o movimento de $u_{2}=R$ por ter maior valor, reduzindo as magnitudes dos movimentos de $R$. 


\section{4.}

\section{Testes de performance dos controladores}

Esta seção apresenta testes realizados de forma a comparar a performance das 3 estratégias de controle estudadas: H-NMPC, NN-NMPC e controle PID tradicional. Foram feitos testes para verificar o rastreamento da trajetória de referência, o desacoplamento das variáveis controladas e a rejeição de distúrbios na vazão e composição da alimentação.

\subsection{1.}

Teste de rastreamento da trajetória de referência e desacoplamento das variáveis controladas

Foram feitos testes de rastreamento para controle dual das trajetórias de referências das composições dos produtos de topo e de fundo da coluna despropanizora para diversos valores de referência, variando-se a amplitude em forma de degrau. Também foi testado o desacoplamento das variáveis controladas, de forma a manter o valor de referência constante para C4D, dando um degrau para C3B.

\section{a) H-NMPC}
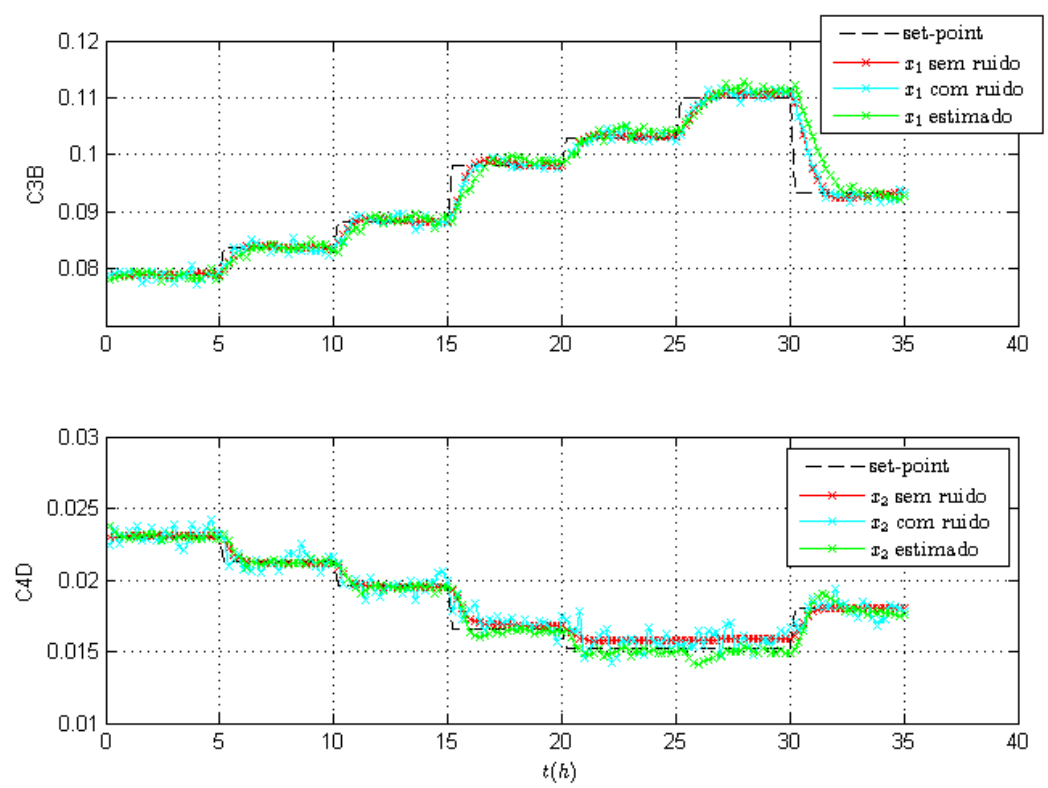

Figura 55: Comparação entre as variáveis estimadas e a trajetória de referência com H-NMPC. 


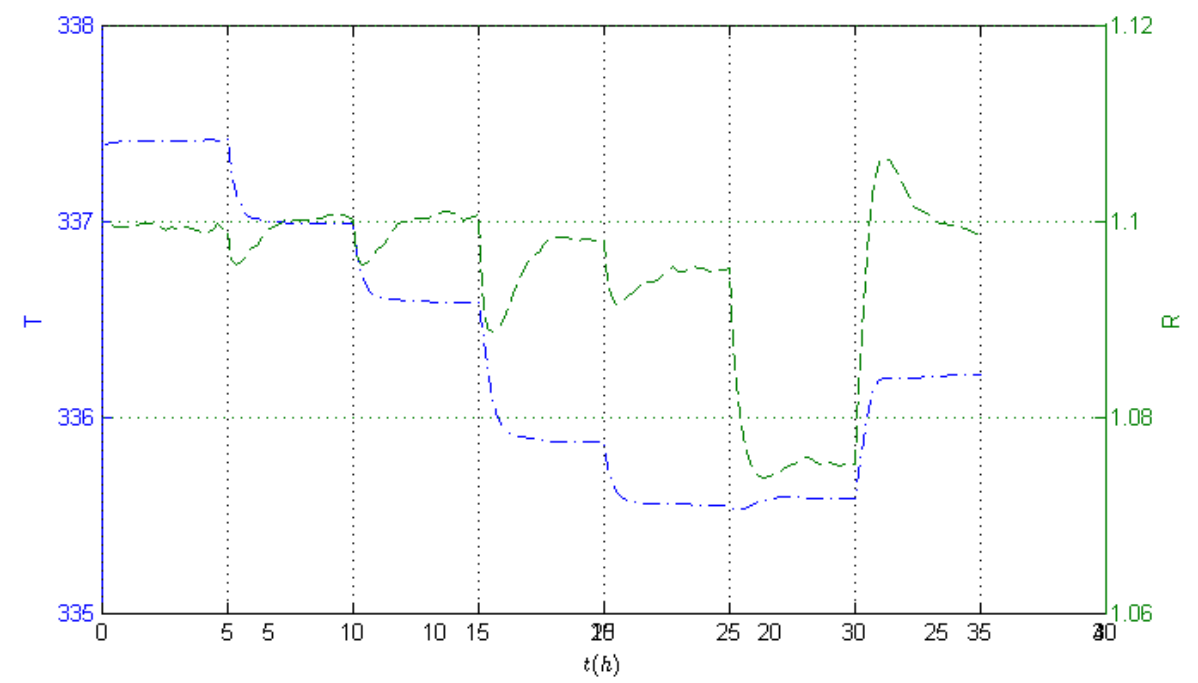

Figura 56: Entradas T e R calculadas para mudança de trajetória das entradas do H-NMPC.

Observa-se na Figura 55 que os valores de referência em diferentes regiões de controle são alcançados, em que o SSE entre as trajetória de referência e as variáveis estimadas foi de $1,9 \cdot 10^{-3}$.

O controlador consegue manter o valor de C4D constante variando o valor de C3B, entre os instantes 25 e 30h, ou seja, foi capaz de desacoplar as variáveis. Controladores PID não são capazes de realizar este desacoplamento. A Figura 56 mostra o comportamento das variáveis manipuladas de forma a manter as variáveis controladas na trajetória de referência.

O tempo necessário para realizar os cálculos do algoritmo foi de 218,4 segundos. 


\section{b) NN-NMPC}
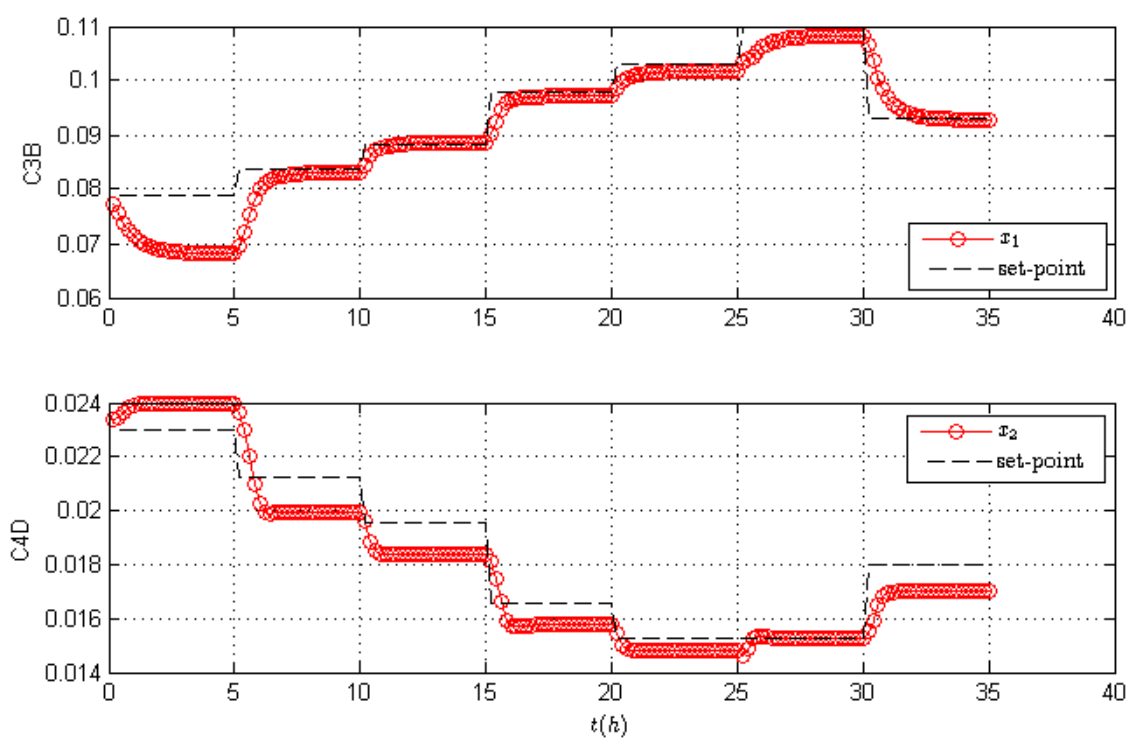

Figura 5714: Comparação entre as variáveis controladas e a trajetória de referência com NN-NMPC.

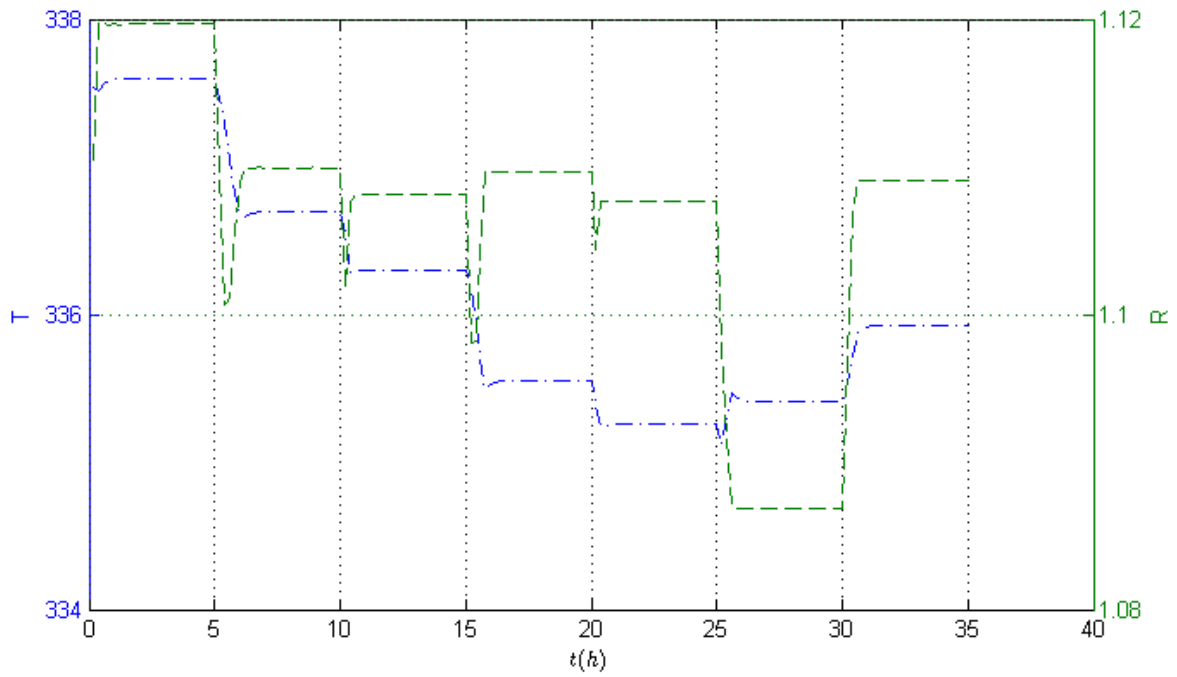

Figura 15: Entradas T e R calculadas para mudança de trajetória das entradas do NN-NMPC.

Observa-se na Figura 57 que os valores de referência em diferentes regiões do controle não foram alcançados satisfatoriamente, em que o SSE entre a trajetória de referência e as variáveis controladas é de $4,3 \cdot 10^{-3}$. Mesmo 
sintonizando os pesos das variáveis controladas e manipuladas, a saída C3B se mantém mais próxima de sua trajetória de referência do que a saída C4D.

Verifica-se que o controlador foi capaz de desacoplar as variáveis controladas entre os instantes 25 e $30 \mathrm{~h}$, em que o valor de C4D foi mantido próximo da trajetória de referência, variando-se o valor de C3B. Apesar do controle para este teste não ter sido satisfatório, houve o desacoplamento.

A primeira trajetória de referência fornecida para as variáveis controladas estava no valor atual da planta simulada, mas desviou da trajetória mesmo assim, normalizando apenas no segundo degrau.

A Figura 58 mostra o comportamento das variáveis manipuladas de forma a manter as variáveis controladas na trajetória de referência.

O tempo necessário para realizar os cálculos do algoritmo foi de 601,1 segundos.

\section{c) PID}
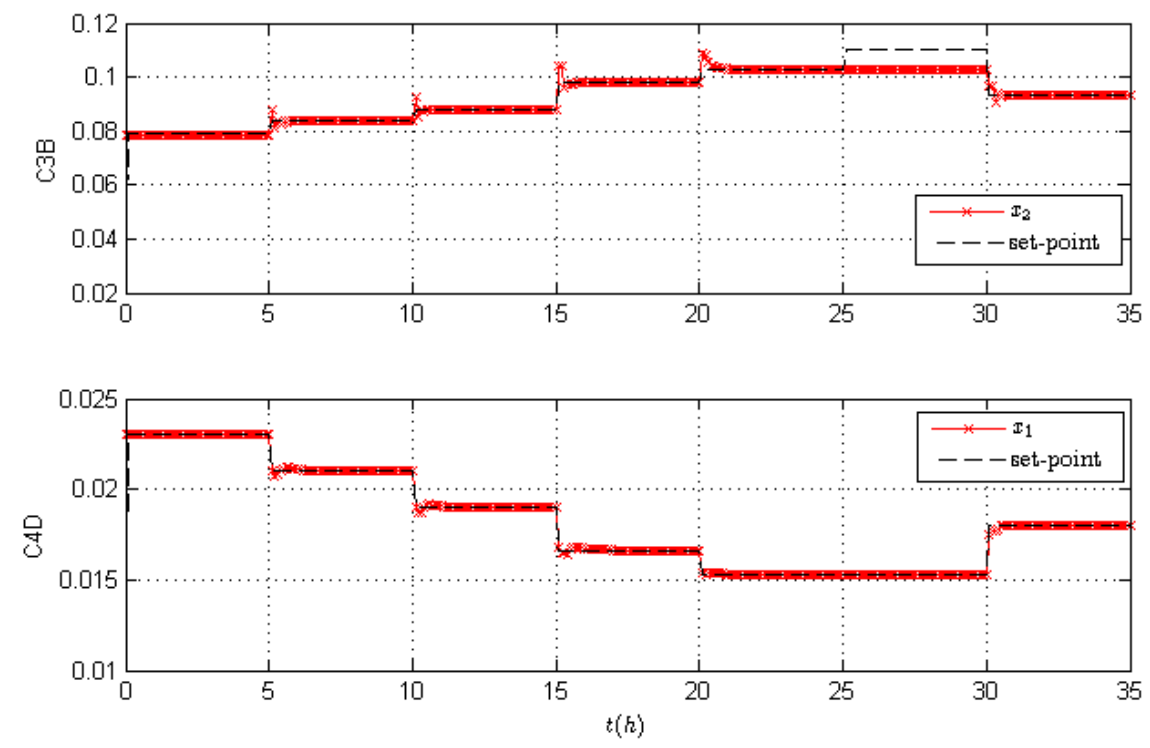

Figura 59: Comparação entre as variáveis controladas e a trajetória de referência com controle regulatório. 


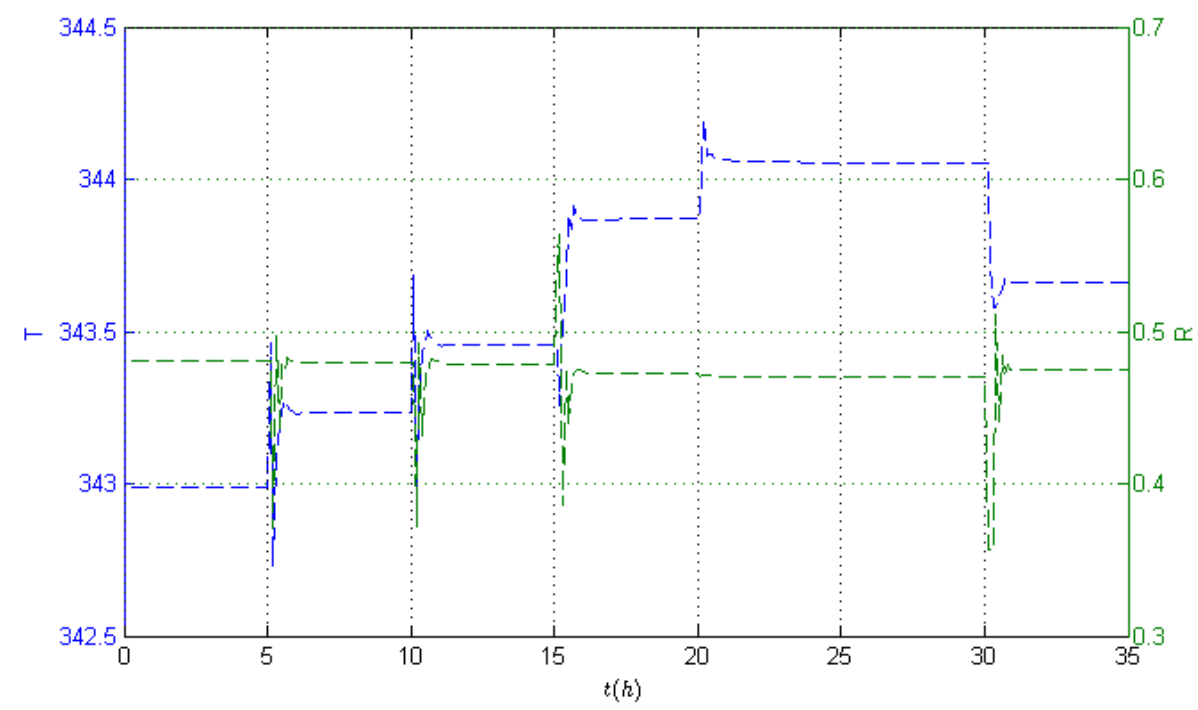

Figura 60: Entradas T e R calculadas para mudança de trajetória das entradas com controle regulatório.

Observa-se na Figura 59 que o valor de referência em diferentes regiões do controle é alcançado dentro da faixa de operação estipulada, porém com overshooting e undershooting, em que o SSE entre a trajetória de referência e as variáveis controladas foi de $7,7 \cdot 10^{-4}$. Porém, mesmo apresentando este comportamento, o controlador foi capaz de rastrear a trajetória de referência mais rapidamente que os demais controladores.

Ao aplicar um degrau no valor de C4D mantendo C3B entre os instantes 25 e 30h, ambos se mantém constantes, não havendo o desacoplamento das variáveis, como esperado.

A Figura 60 mostra o comportamento das variáveis manipuladas de forma a manter as variáveis controladas na trajetória de referência.

O tempo necessário para realizar os cálculos do algoritmo foi de 182,5 segundos. 


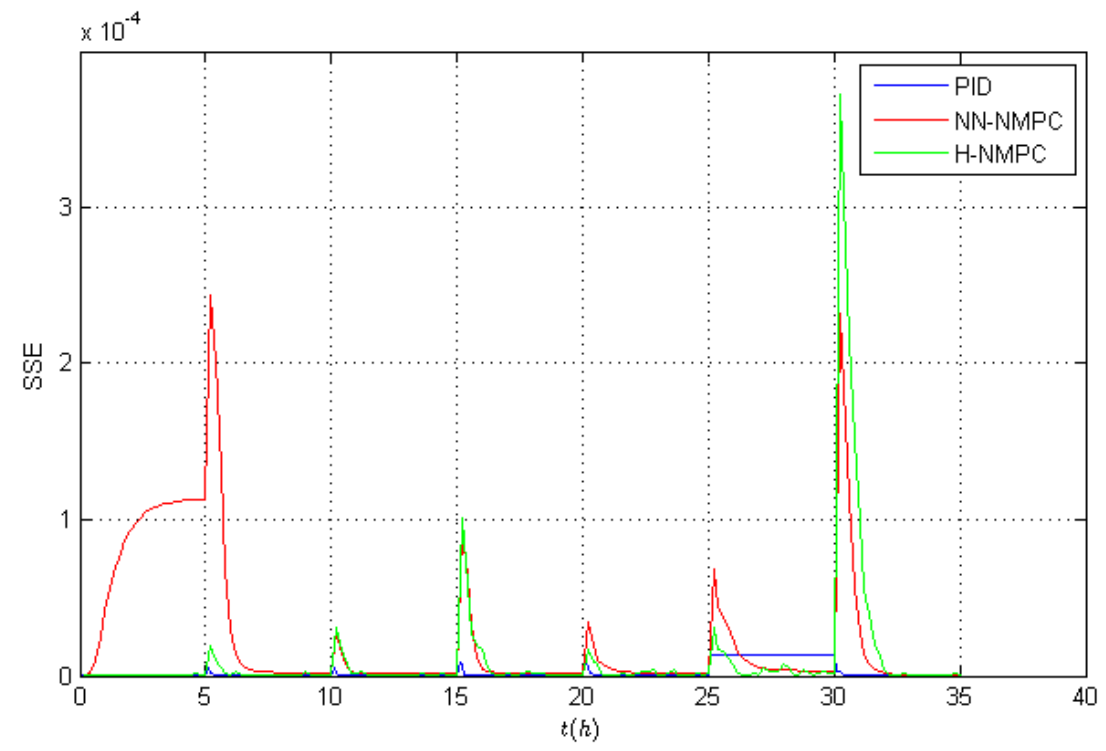

Figura 61: Soma dos erros quadráticos dos controladores H-NMPC, NN-NMPC e PID para teste de rastreamento de trajetória de referência.

A Figura 61 apresenta o SSE entre as saídas dos controladores H-NMPC, NN-NMPC e PID e a saída desejada para teste de rastreamento de trajetória de referência. Observa-se um erro inicial grande para o NN-NMPC e um erro constante para PID entre 25 e 30h, em que o controle não consegue desacoplar as variáveis. Observa-se também nos demais picos referentes a alterações de setpoint que o PID apresenta menor SSE, pois, apesar de apresentar overshooting, ele foi capaz de rastrear a trajetória de referência mais rapidamente que os demais controladores. O H-NMPC apresentou bom rastreamento de trajetória de referência desde o início da simulação e foi capaz de desacoplar as variáveis, indicando ser o controlador com melhor desempenho para este teste.

\subsection{2.}

\section{Teste de rejeição de Distúrbios}

Nesta seção, são apresentados os resultados obtidos para a rejeição de distúrbios na vazão e composição da alimentação para os controladores H-NMPC, NN-NMPC e PID. 


\subsubsection{Vazão da alimentação}

Neste teste, foi aplicado um distúrbio de 10\% na vazão da alimentação da coluna de destilação, variando-se a amplitude em forma de degrau, para observar a controlabilidade dos controladores para tentar manter o setpoint dentro da especificação, alterando a vazão de $1 \mathrm{kmol} / \mathrm{s}$ para $1,1 \mathrm{kmol} / \mathrm{s}$.

\section{a) H-NMPC}
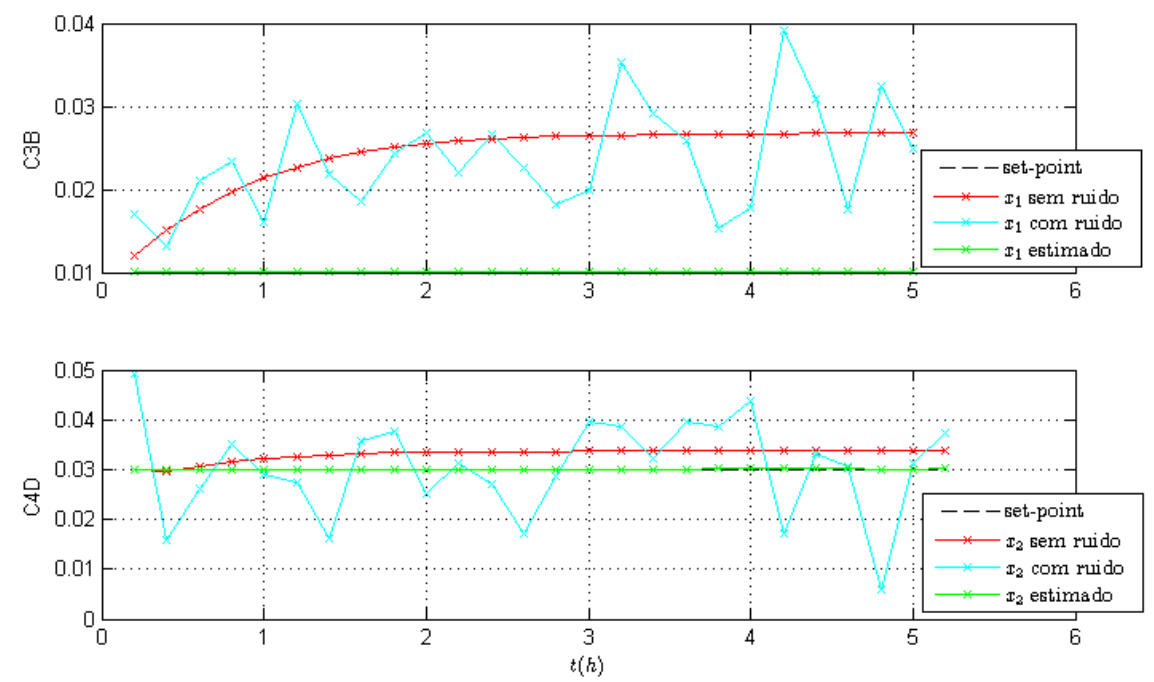

Figura 62: Efeito nas saídas causado pelo distúrbio aplicado na vazão de alimentação com H-NMPC.
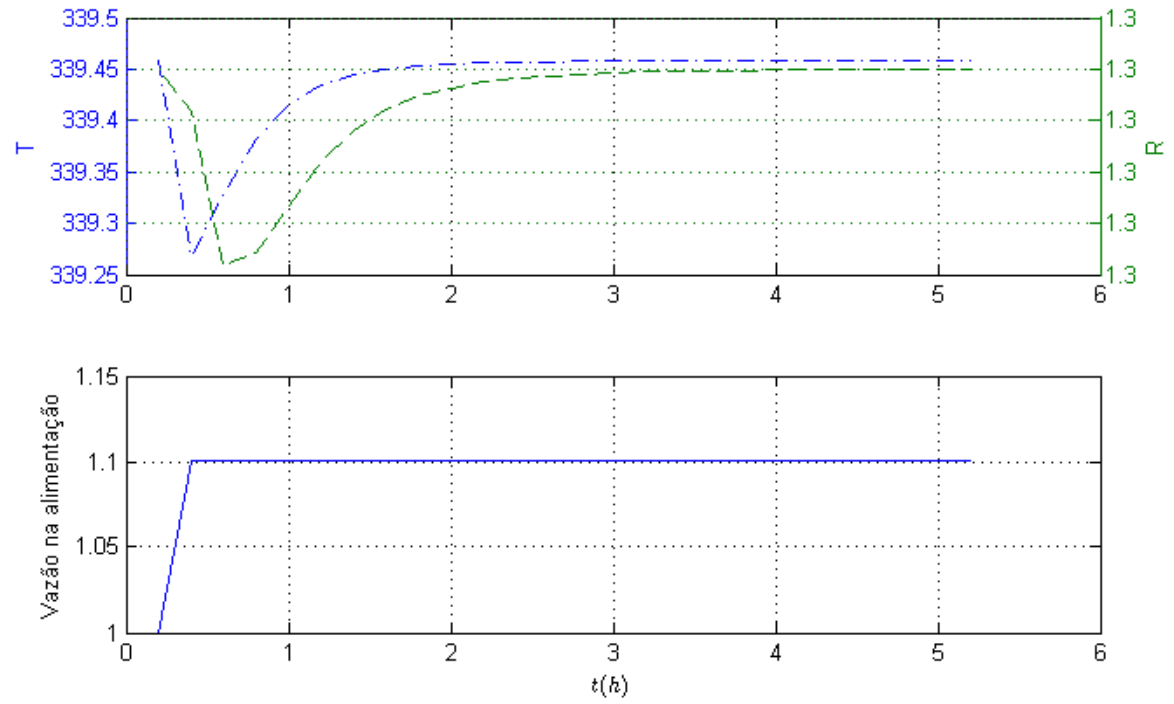

Figura 63: Distúrbio em degrau na vazão de alimentação e entradas calculadas com H-NMPC. 
A Figura 62 mostra o efeito nas saídas causado pelo distúrbio aplicado na vazão de alimentação. Os valores das variáveis controladas com ruído saem da especificação desejada após aplicação do distúrbio, em que C3B chega a aproximadamente 0,027 e C4D a 0,034. Porém, as variáveis estimadas estão bem próximas da especificação desejada, resultando em um bom desempenho do controlador. O SSE entre a trajetória de referência e as variáveis estimadas foi de $3,8 \cdot 10^{-7}$.

A Figura 63 mostra o distúrbio em degrau aplicado à vazão de alimentação e as entradas calculadas com H-NMPC.

\section{b) NN-NMPC}
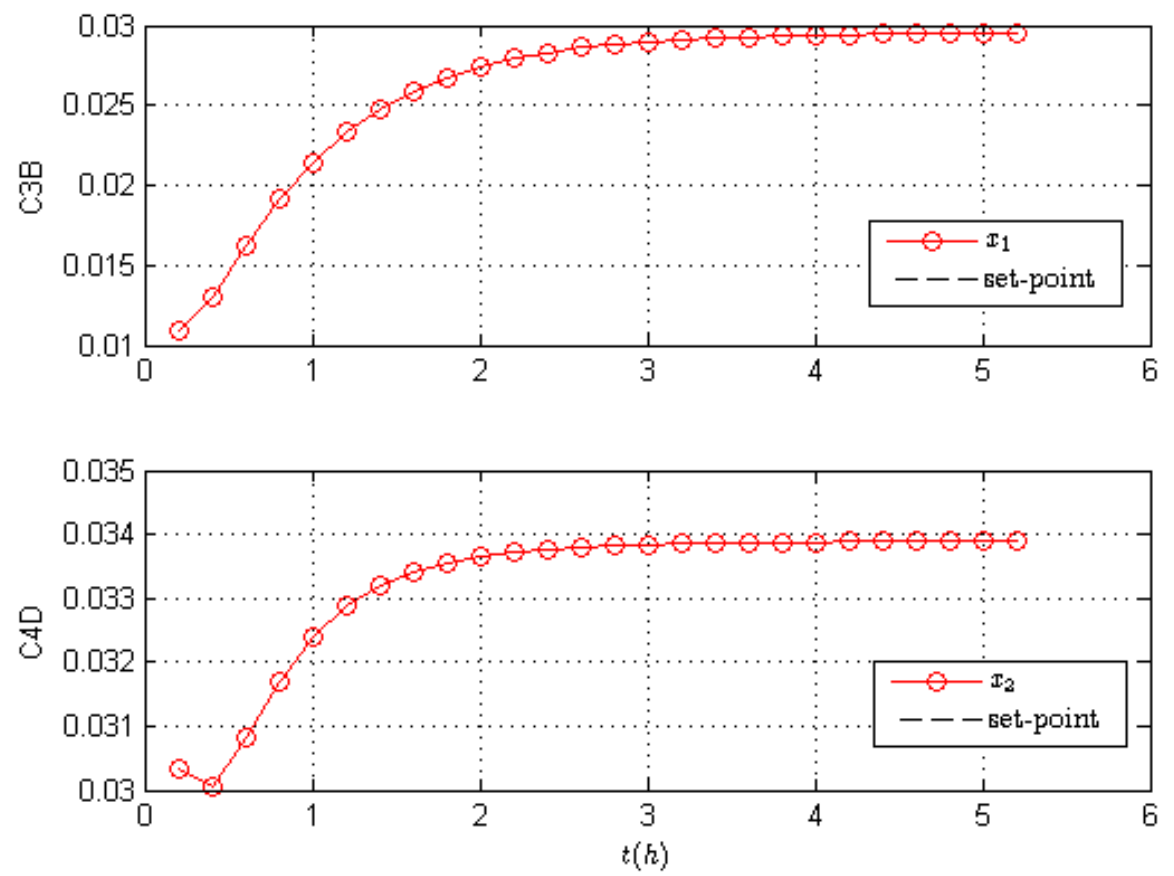

Figura 64: Efeito nas saídas causado pelo distúrbio aplicado na vazão de alimentação com NN-NMPC. 

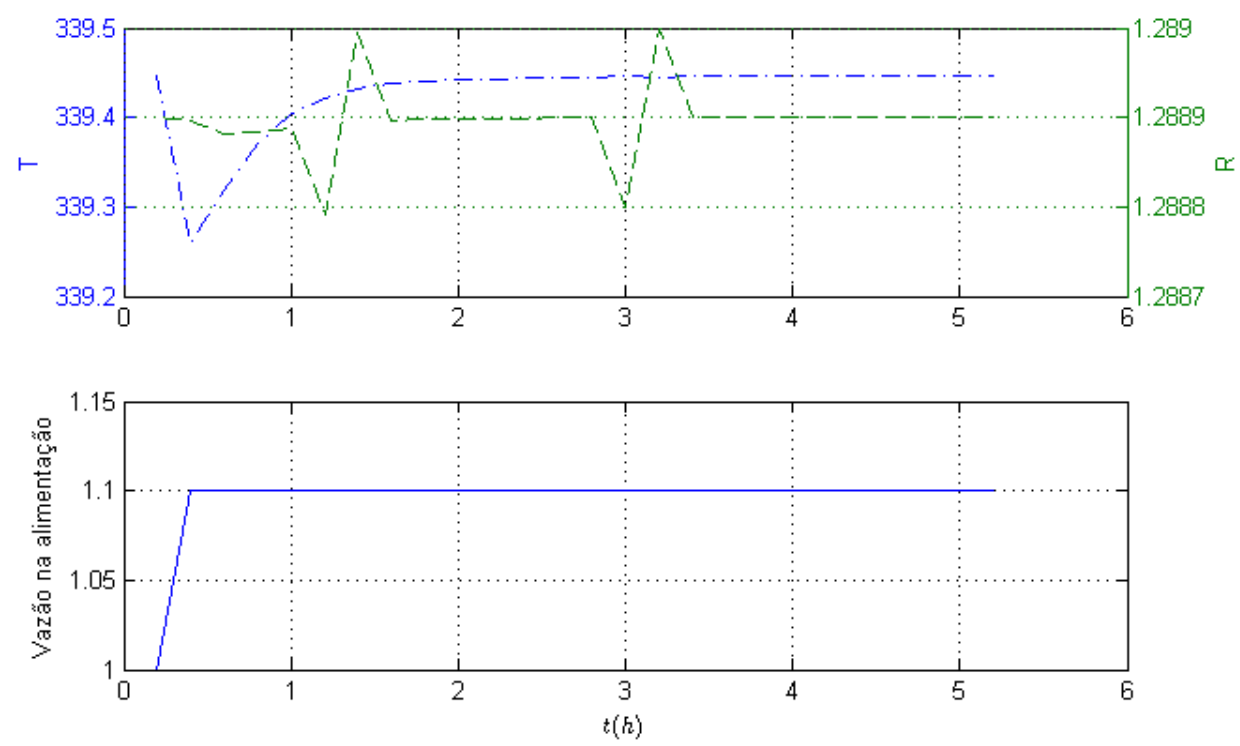

Figura 65: Distúrbio em degrau na vazão de alimentação e entradas calculadas com NN-NMPC.

A Figura 64 mostra o efeito nas saídas causado pelo distúrbio aplicado na vazão de alimentação. Os valores das variáveis controladas saem da especificação desejada após aplicação do distúrbio, se comportando da mesma forma que as variáveis controladas com ruído teste de distúrbio do H-NMPC, com C3B a 0,03 e C4D a 0,034. O controlador não foi capaz de manter as variáveis dentro da especificação, logo, este não demonstrou bom desempenho. O SSE entre a trajetória de referência e as variáveis estimadas foi de $7,3 \cdot 10^{-3}$.

A Figura 65 mostra o distúrbio em degrau aplicado à vazão de alimentação e as entradas calculadas com NN-NMPC. 


\section{c) PID}
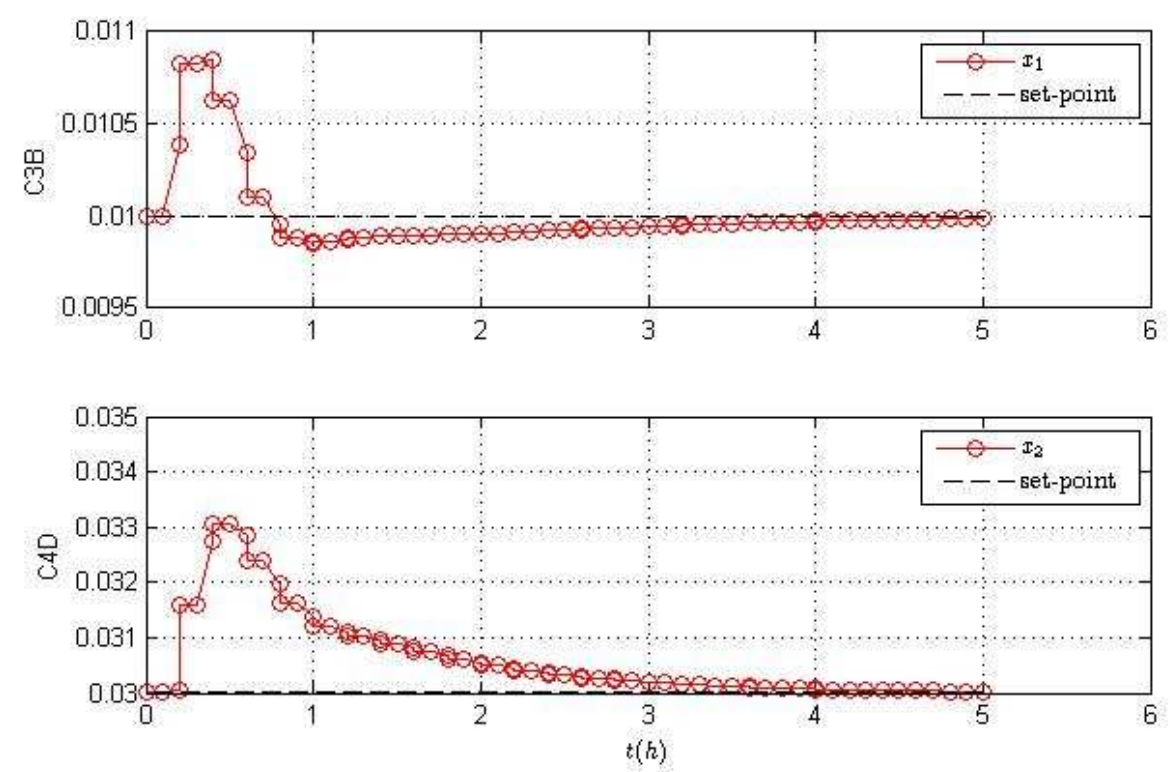

Figura 66: Efeito nas saídas causado pelo distúrbio aplicado na vazão de alimentação com controlador PID.
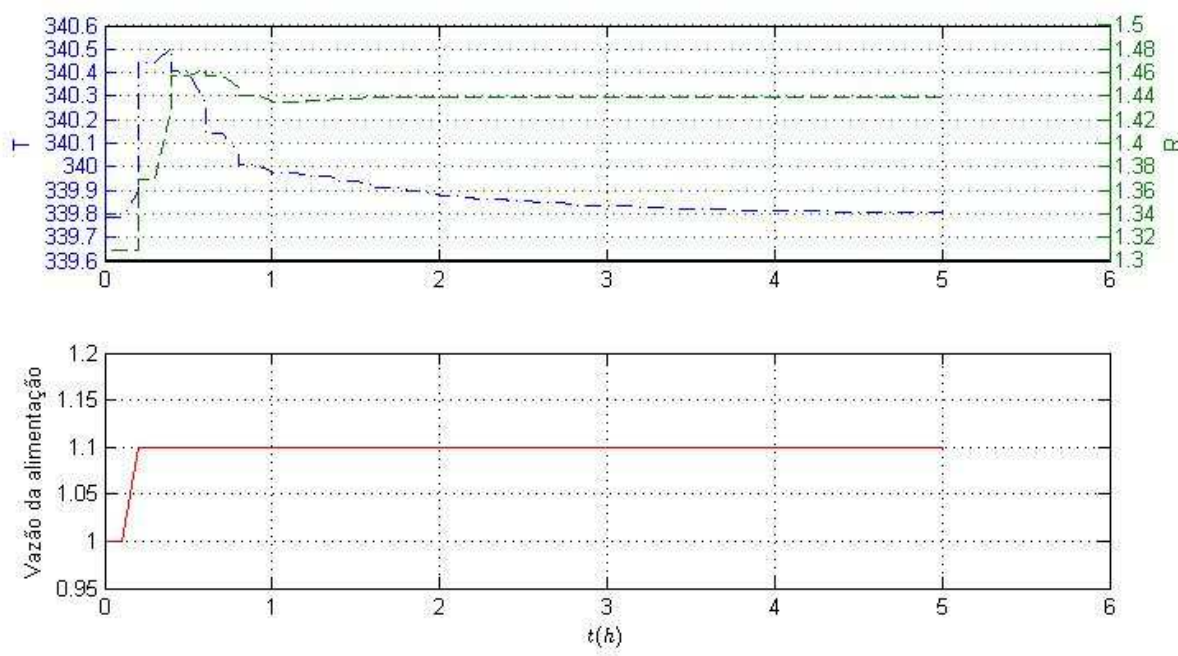

Figura 67: Distúrbio em degrau na vazão de alimentação e entradas calculadas com controlador PID.

A Figura 66 mostra o efeito nas saídas causado pelo distúrbio aplicado na vazão de alimentação. Os valores das variáveis controladas apresentam um overshooting, com amplitude de aproximadamente $10 \%$ do valor das especificações, com C3B atingindo até 0,011 e C4D até 0,033. Após 
aproximadamente 3h após o distúrbio, o controlador conseguiu restabelecer as composições dentro das especificações desejadas. O SSE entre a trajetória de

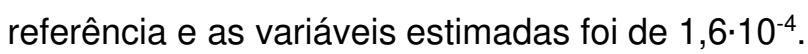

A Figura 67 mostra o distúrbio em degrau aplicado à vazão de alimentação e as entradas calculadas com controlador PID.

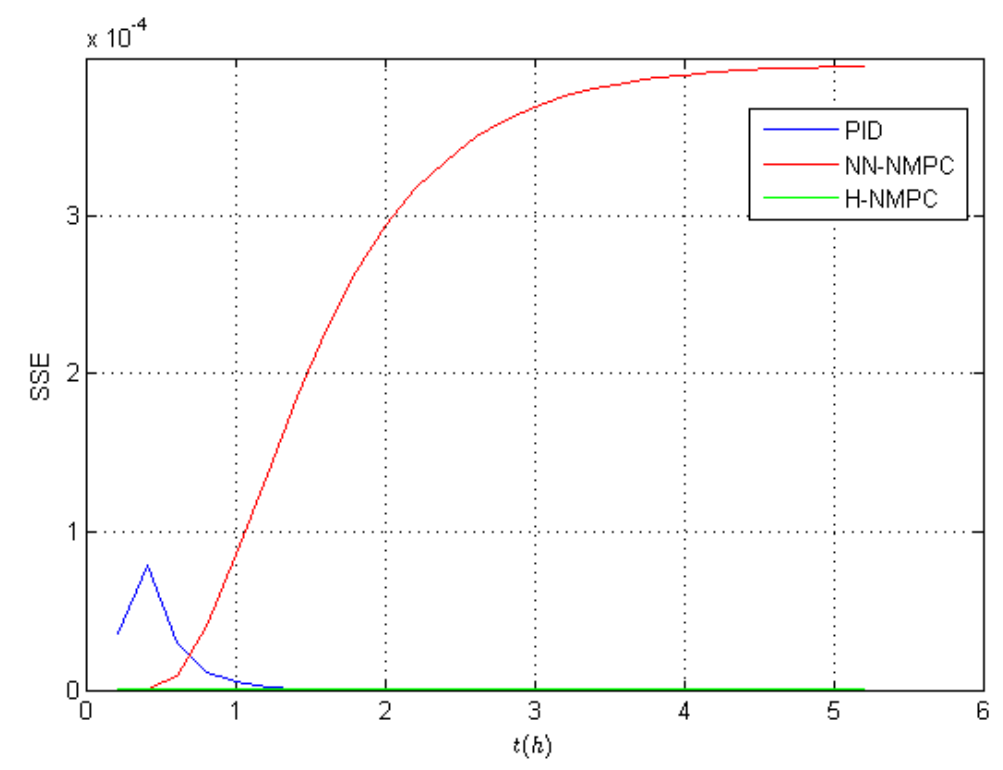

Figura 68: Soma dos erros quadráticos dos controladores H-NMPC, NN-NMPC e PID para teste de distúrbio da vazão da alimentação.

A Figura 68 apresenta a soma do erro quadrático a cada instante entre as saídas dos controladores H-NMPC, NN-NMPC e PID e a saída desejada para teste de distúrbio da vazão da alimentação. Observa-se para o NN-NMPC, um erro constante após o distúrbio. Para o controlador PID, observa-se um pico no início do distúrbio e para H-NMPC erro menor e constante em relação aos outros controladores, sugerindo ser o controlador com melhor performance para este teste.

\subsubsection{Composição da alimentação}

Para observar a controlabilidade dos controladores para tentar manter o setpoint dentro da especificação, foi aplicado um distúrbio de $10 \%$ na composição de propano da alimentação da coluna de destilação, variando-se a amplitude em forma de degrau, aumentando a fração molar de C3 de 0,40 para 0,44 e diminuindo a do $\mathrm{C} 4$ de 0,60 para 0,56 . 


\section{a) H-NMPC}
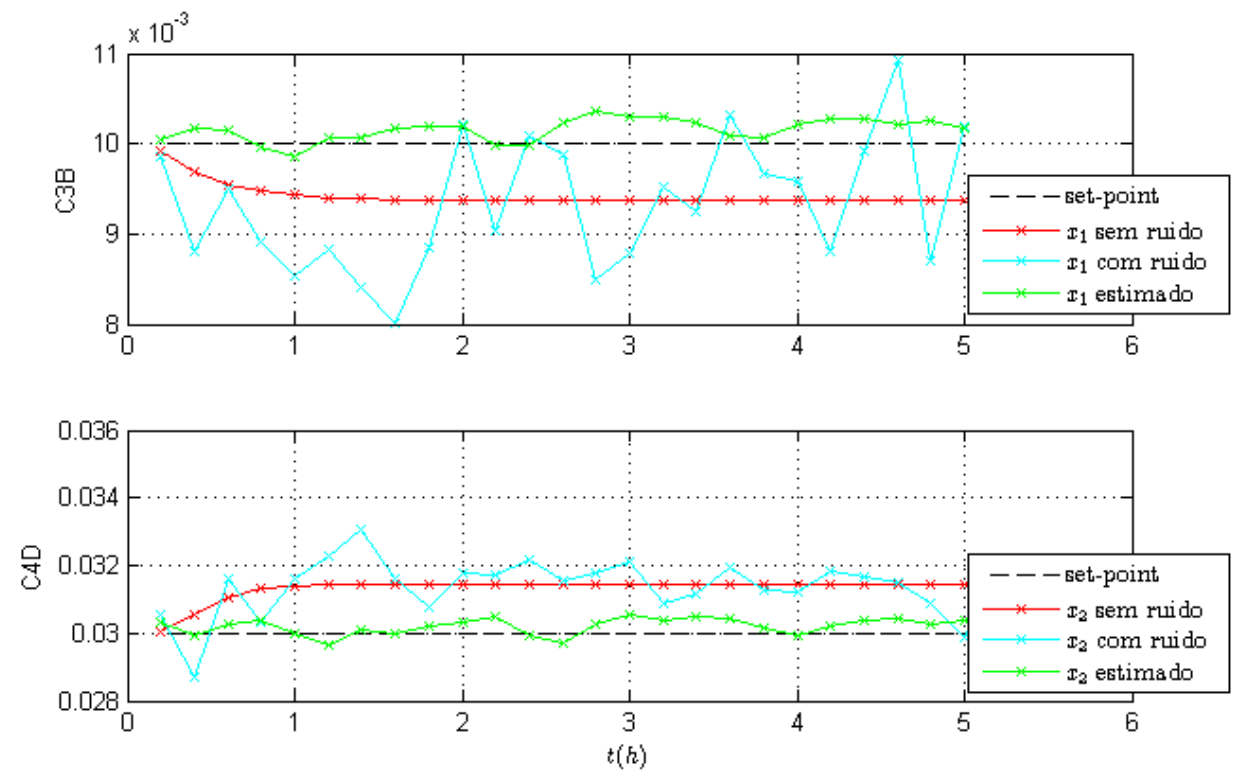

Figura 69: Efeito nas saídas causado pelo distúrbio aplicado na composição da alimentação com H-NMPC.
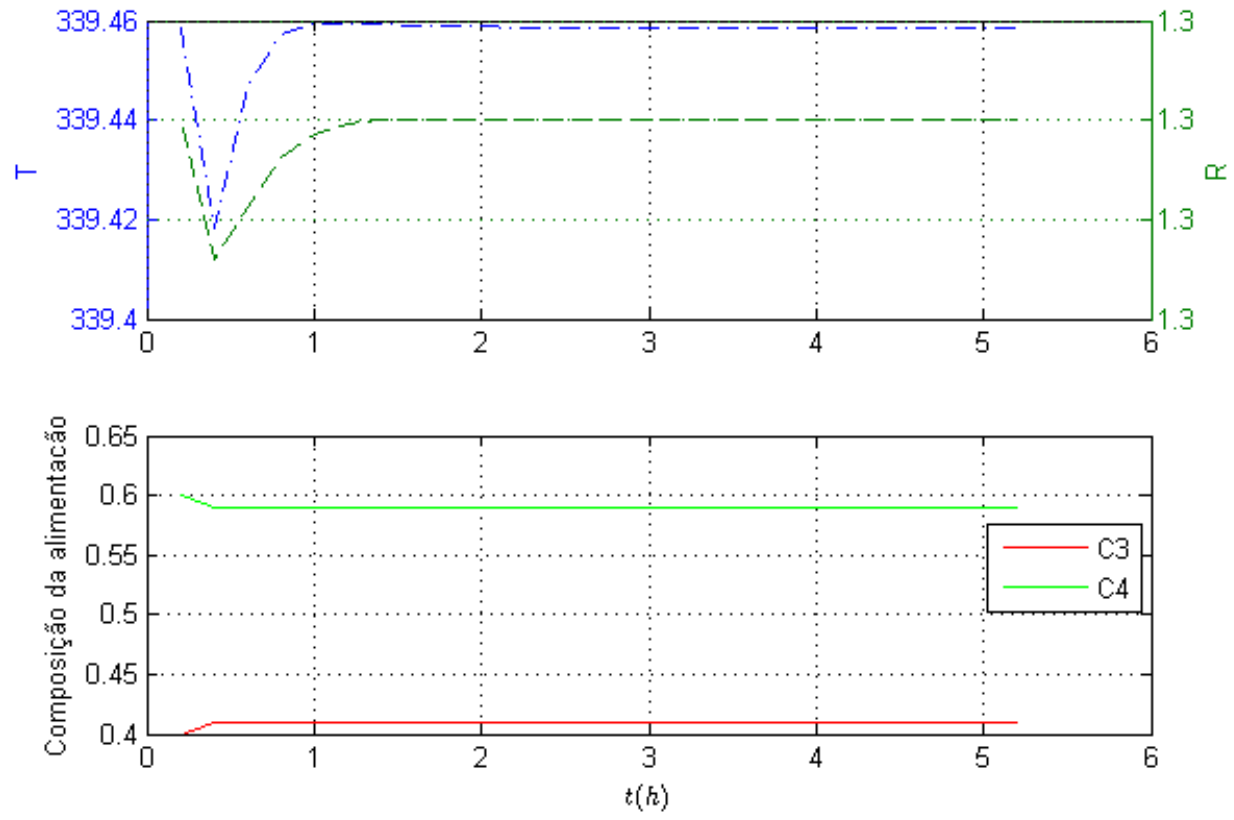

Figura 70: Distúrbio em degrau na composição da alimentação e entradas calculadas com H-NMPC.

A Figura 69 mostra o efeito nas saídas causado pelo distúrbio aplicado na composição da alimentação. Os valores das variáveis controladas com ruído saem da especificação desejada após aplicação do distúrbio, em que C3B chega a 
aproximadamente 0,0095 e C4D a 0,0315. Porém, as variáveis estimadas estão bem próximas da especificação desejada, resultando em um bom desempenho do controlador. O SSE entre a trajetória de referência e as variáveis controladas estimadas foi de $4,5 \cdot 10^{-6}$.

A Figura 70 mostra o distúrbio em degrau aplicado à composição da alimentação e as entradas calculadas com H-NMPC.

\section{b) NN-NMPC}
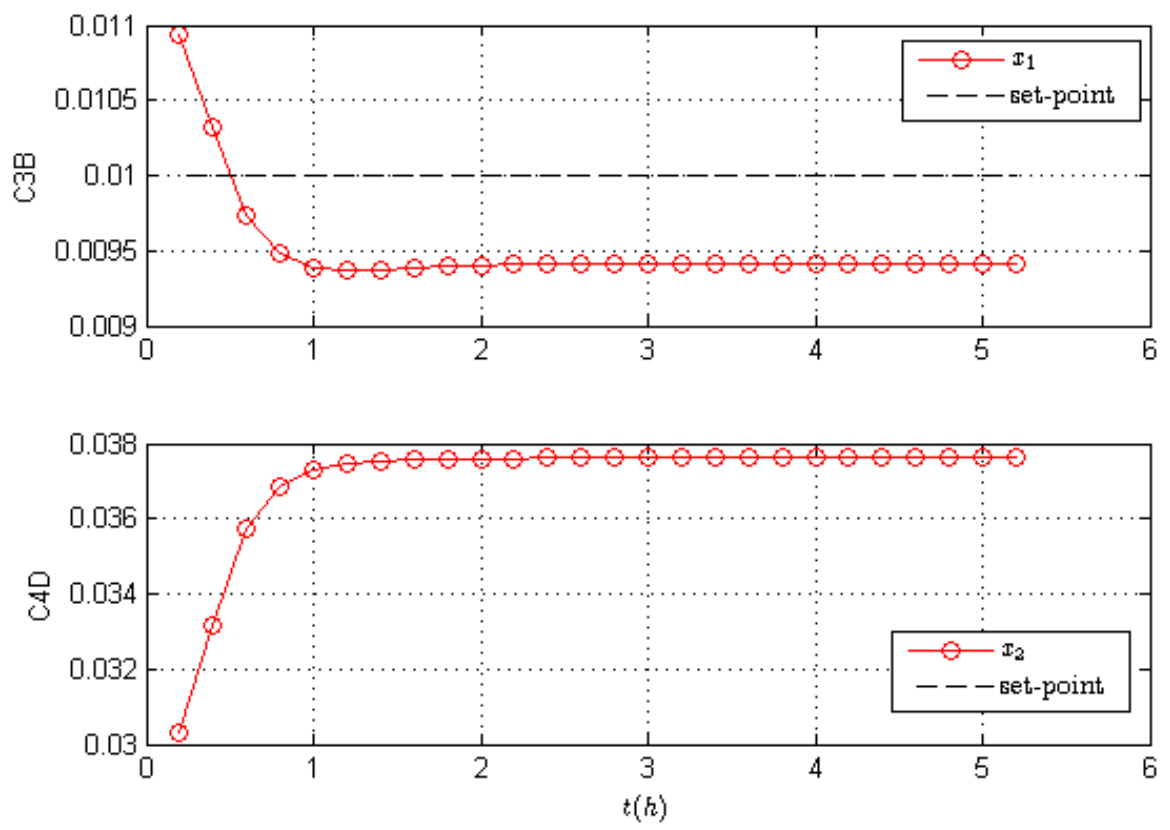

Figura 71: Efeito nas saídas causado pelo distúrbio aplicado na composição de alimentação com NN-NMPC. 

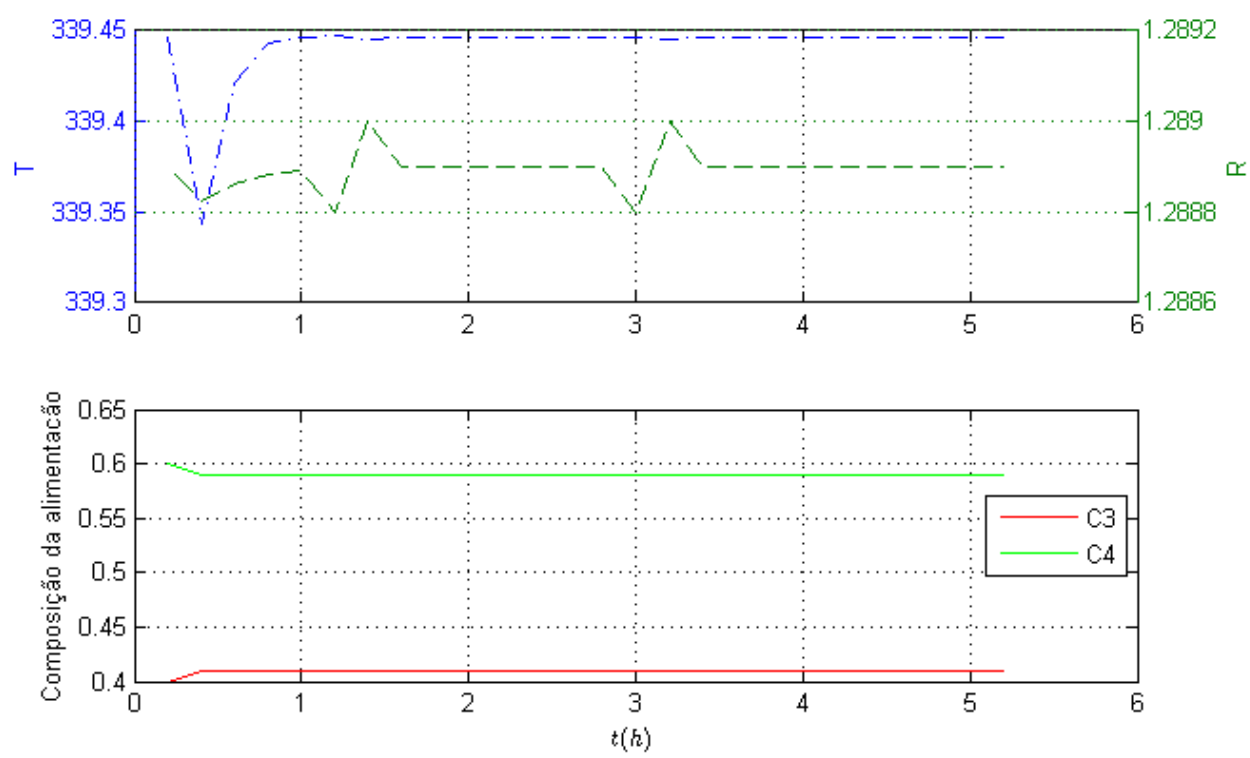

Figura 72: Distúrbio em degrau na composição da alimentação e entradas calculadas com NN-NMPC.

A Figura 71 mostra o efeito nas saídas causado pelo distúrbio aplicado na composição da alimentação. Os valores das variáveis controladas saem da especificação desejada após aplicação do distúrbio, em que C3B chega a aproximadamente 0,0095 e C4D a 0,038, não apresentando um bom desempenho do controlador. O SSE entre a trajetória de referência e as variáveis controladas foi de $1,5 \cdot 10^{-3}$.

A Figura 72 mostra o distúrbio em degrau aplicado à composição da alimentação e as entradas calculadas com NN-NMPC 


\section{c) PID}
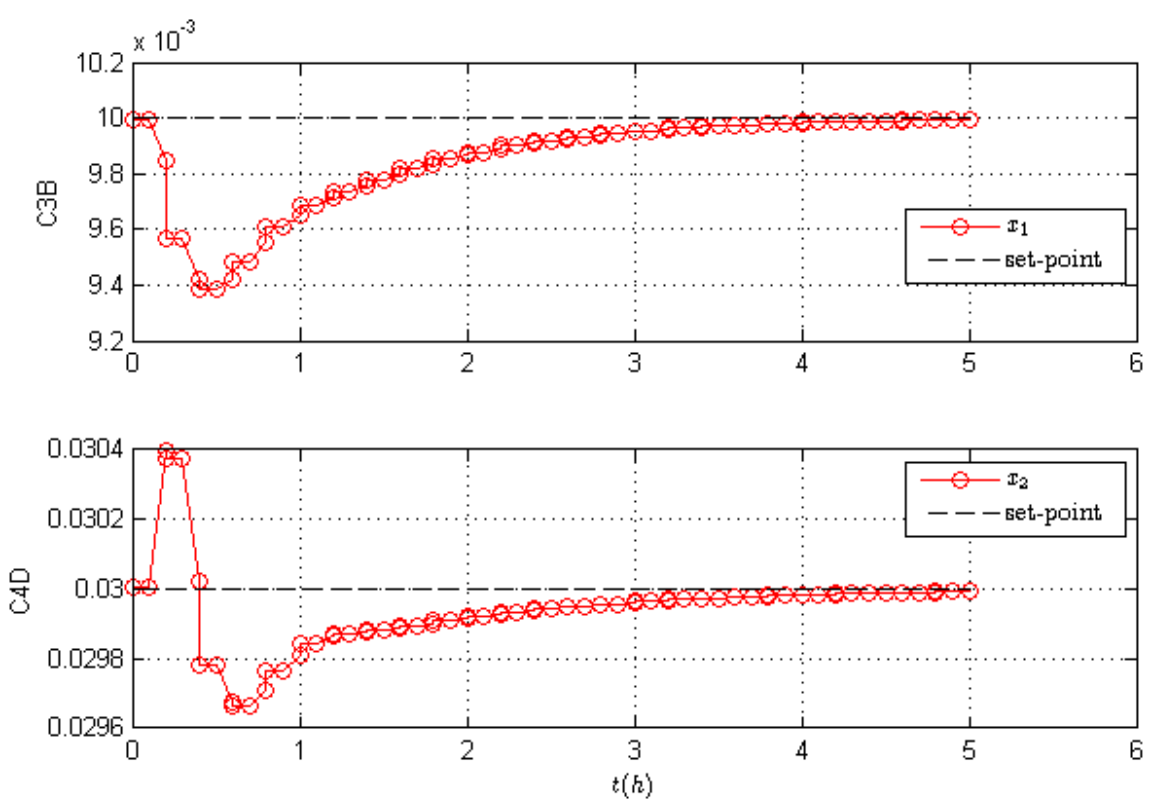

Figura 73: Efeito nas saídas causado pelo distúrbio aplicado na composição de alimentação com controlador PID.
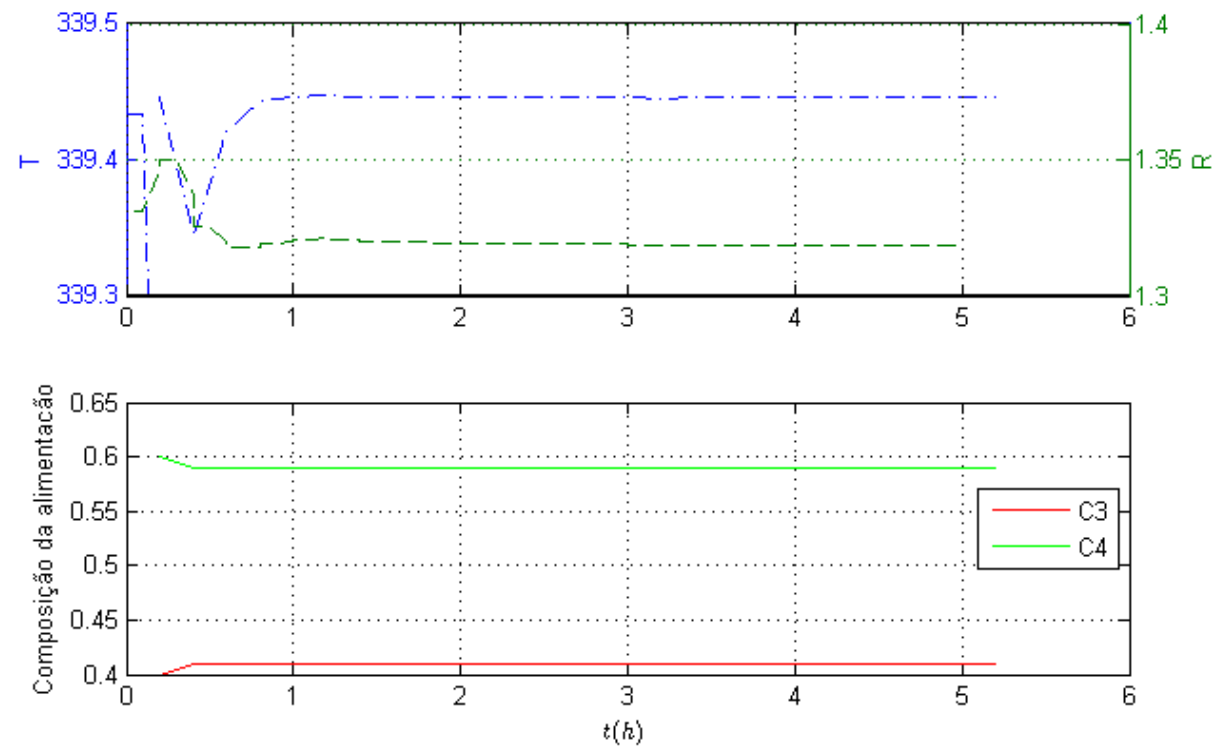

Figura 74: Distúrbio em degrau na composição da alimentação e entradas calculadas com controlador PID.

A Figura 73 mostra o efeito nas saídas causado pelo distúrbio aplicado na vazão de alimentação. Os valores das variáveis controladas apresentam um overshooting, com C3B atingindo até 0,0094 e C4D oscilando de 0,0304 a 0,0296, 
porém a amplitude é baixa. Após aproximadamente 3h após o distúrbio, 0 controlador conseguiu restabelecer as composições dentro das especificações desejadas. O SSE entre a trajetória de referência e as variáveis estimadas foi de $5,3 \cdot 10^{-6}$.

A Figura 74 mostra o distúrbio em degrau aplicado à composição da alimentação e as entradas calculadas com controlador PID.

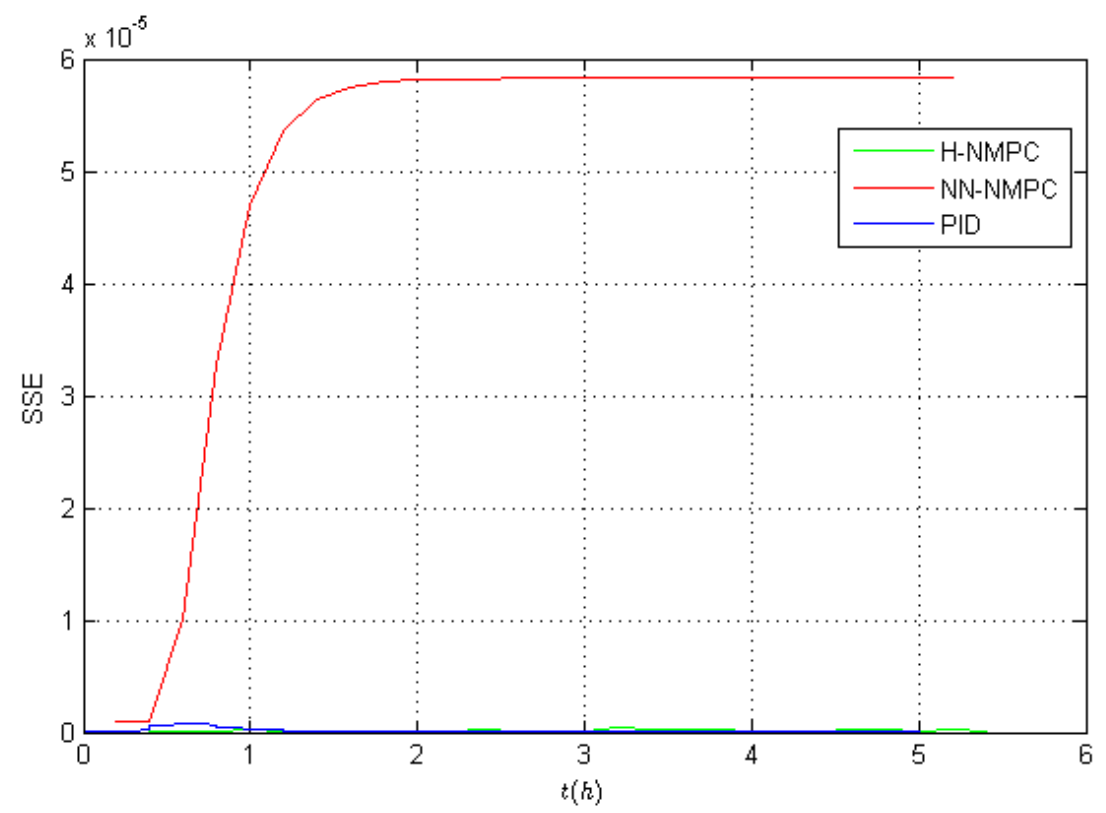

Figura 75: Soma dos erros quadráticos dos controladores H-NMPC, NN-NMPC e PID para teste de distúrbio da composição da alimentação.

A Figura 75 apresenta a soma do erro quadrático a cada instante entre as saídas dos controladores H-NMPC, NN-NMPC e PID e a saída desejada para teste de distúrbio da composição da alimentação. Observa-se para o NN-NMPC, um erro constante após o distúrbio. Para o controlador PID, observa-se um pico no início do distúrbio. O H-NMPC tem erro menor e constante em relação aos outros controladores, sugerindo ser o controlador com melhor performance para este teste.

\subsubsection{Resumo dos resultados dos testes}

A Tabela 14 apresenta um resumo dos resultados dos testes. As duas primeiras linhas mostram o tempo necessário para realizar os cálculos do algoritmo dos controladores e o SSE para os testes de rastreamento das 
trajetórias. As linhas seguintes mostram os valores de SSE para os testes de distúrbios aplicados na vazão e composição da alimentação.

Tabela 84: Resumo dos resultados qualitativos dos testes.

\begin{tabular}{|c|c|c|c|}
\cline { 2 - 4 } \multicolumn{1}{c|}{} & Hammerstein & Redes Neurais & PID \\
\hline tempo (s) & 218,4 & 601,1 & 182,5 \\
\hline SSE do rastreamento & $1,9 \cdot 10^{-3}$ & $4,3 \cdot 10^{-3}$ & $7,7 \cdot 10^{-4}$ \\
\hline SSE da vazão da alimentação & $3,8 \cdot 10^{-7}$ & $7,3 \cdot 10^{-3}$ & $1,6 \cdot 10^{-4}$ \\
\hline SSE da composição da alimentação & $4,5 \cdot 10^{-6}$ & $1,5 \cdot 10^{-3}$ & $5,3 \cdot 10^{-6}$ \\
\hline
\end{tabular}

\section{5.}

\section{Discussão dos resultados}

O tempo necessário para realizar os cálculos do algoritmo de cada um dos controladores no teste de rastreamento da trajetória foi de $218,4 \mathrm{~s}$ para $0 \mathrm{H}$ NMPC, de $601,1 \mathrm{~s}$ para o NN-NMPC e de $182,5 \mathrm{~s}$ para o controle PID tradicional. Era esperado o controlador PID ser o mais rápido, por ser baseado em singleloops locais. Isso se deve ao fato do cálculo das trajetórias de referência ser feito individualmente para cada variável.

O H-NMPC foi cerca de três vezes mais rápido que o NN-NMPC, devido à rapidez dos cálculos matriciais do modelo. Além disso, para H-NMPC, a formulação da função de custo foi feita de forma analítica, permitindo o uso do gradiente e da hessiana para acelerar o tempo de otimização. Já para o NN-MPC, não foi possível utilizar a forma analítica da função de custo devido à complexidade do modelo de redes neurais, portanto a forma numérica da função foi utilizada.

Para o teste de rastreamento da trajetória de referência, o controle PID se mostrou com menor SSE, porém apresentou overshooting e undershooting a cada degrau dado. Este comportamento dinâmico não é desejado para colunas de destilação, pois a presença de oscilações na planta aumentam a variabilidade das variáveis de processo causando mau desempenho de controle, diminuindo a qualidade dos produtos e diminuindo a lucratividade da planta. Em geral, desejase um comportamento super-amortecido para a resposta do sistema. O H-NMPC mostrou melhor desempenho para este teste, com SSE $=1,9 \cdot 10^{-3} \mathrm{e}$ comportamento super-amortecido das respostas.

Para o teste de desacoplamento das variáveis controladas, ambos os controladores NMPC foram capazes de fazer o desacoplamento, porém $\circ \mathrm{H}$ - 
NMPC teve melhor desempenho para o teste. O controlador PID não foi capaz de desacoplar as variáveis.

Para o teste de distúrbio na vazão da alimentação, o NN-NMPC não foi capaz de manter as variáveis controladas dentro das especificações. O controlador PID apresentou oscilação novamente, voltando a controlar as variáveis controladas após 3h. O H-NMPC foi capaz de manter as variáveis dentro da especificação sem oscilação, com SSE $=4,5 \cdot 10^{-6}$, demonstrando melhor desempenho para este teste.

Para o teste de distúrbio na composição da alimentação, os controladores tiveram respostas parecidas para o teste de distúrbio na vazão da alimentação, em que o controlador PID apresentou comportamento oscilatório, o NN-NMPC não foi capaz de manter as variáveis controladas dentro das especificações e $\circ \mathrm{H}$ NMPC foi capaz de manter as variáveis dentro da especificação sem oscilação, com SSE $=5,3 \cdot 10^{-6}$, demonstrando melhor desempenho para este teste. 


\section{5.}

\section{Conclusão}

Este trabalho propôs o estudo de três estruturas de controle aplicadas a uma coluna despropanizadora: controle PID tradicional, H-NMPC e NN-NMPC. Os testes realizados para verificar o desempenho de cada controlador foram rastrear diferentes trajetórias de referência, a desacoplar as variáveis controladas e a rejeitar os distúrbios aplicados na alimentação da coluna.

A estratégia de controle H-NMPC teve excelente desempenho para todos os testes aplicados. Este teve os melhores desempenhos no rastreamento das trajetórias de referência e no desacoplamento das variáveis controladas, com SSE na ordem de $10^{-3}$. Para os testes de distúrbios, o controlador foi capaz de atenuar os ruídos de medição através do estimador de estado e manteve as variáveis estimadas próximas aos valores de referência, com valores de SSE na ordem de $10^{-6}$ e $10^{-7}$ para teste de distúrbio de vazão e composição da alimentação, respectivamente. Além disso, este controlador apresentou maior rapidez computacional comparado com o NN-NMPC.

Os controladores NN-NMPC e PID apresentaram algumas deficiências nos testes. O NN-NMPC não conseguiu fazer um bom rastreamento das trajetórias de referência e não foi capaz de atenuar ruídos de medição. Este foi capaz de desacoplar as variáveis controladas, mas com desempenho insatisfatório. $O$ controlador PID apresentou overshooting e undershooting a cada degrau dado para rastreamento das trajetórias de referência, sendo este comportamento dinâmico não aceitável para colunas de destilação. Este também não foi capaz de desacoplar as variáveis controladas.

A aplicação do H-NMPC para o controle multivariável de uma coluna despropanizadora, é uma contribuição original para a literatura. Os resultados obtidos são bastante promissores tendo-se em vista o seu desempenho comparado às outras estratégias de controle estudadas. 
6.

\section{Trabalhos futuros}

Sugere-se como trabalho futuro:

- Formulação de um novo modelo de redes neurais para aplicação de NNNMPC, com entradas nos instantes atuais e passados;

- Aplicação do modelo de Hammerstein em que o modelo estacionário nãolinear é modelado com redes neurais;

- Inclusão de otimização em tempo real formulada com base em uma função de lucro da planta e a comparação com abordagens de self-optimizing control. 


\section{7.}

\section{Referências}

AGGOUNE, L.; CHETOUANI, Y. Modeling of a distillation column based on NARMAX and Hammerstein models. International Journal of Modeling, Simulation, and Scientific Computing, Vol. 8, No. 3, 2017.

ABDULLAH, Z.; AZIZ, N.; AHMAD, Z. Nonlinear Modelling Application in Distillation Column. Chemical Product and Process Modeling, 2007.

BARALDI, A. Analise, modelagem e otimizacao do ciclo de refrigeracao de uma unidade de processamento de gas natural. Dissertação (Mestrado em Engenharia Química) - Escola de Engenharia, Universidade Federal do Rio Grande do Sul, Porto Alegre, 2015.

BIAGIOLA, S. I.; FIGUEROA, J. L. Identification of uncertain MIMO Wiener and Hammerstein models. Computers and Chemical Engineering, 2011.

CAMACHO, E. F.; ALBA, C. B. Model predictive control. Springer, 2013.

CAMPOS, M.C.M.M. DE; TEIXEIRA, H.C.G. Controles típicos de equipamentos e processos industriais. $1^{\text {a }}$ Ed. São Paulo: Blucher, 2006.

CHETOUANI, Y. Using Artificial Neural networks for the modelling of a distillation column. Journal of Computer Science \& Applications, Vol. 4 Issue 3, pp 119-133, 2007.

CLARKE, D. W.; MOHTADI, C.; TUFFS, P. S. Generalized predictive control Part I. The basic algorithm. Automatica, v. 23, n. 2, p. 137-148, 1987.

CNI. Gás natural: mercado e competitividade. Confederação Nacional da Indústria, Brasília, 2018.

DARBY, M. et al. RTO: An overview and assessment of current practice. Journal of Process Control, 2011.

DING, Y. et al. Model predictive control and its application in agriculture: $\mathbf{A}$ review. Computers and Electronics in Agriculture, Elsevier, 2018.

GIWA, A.; GIWA, S. O. Layer-Recurrent Neural Network Modelling of Reactive Distillation Process. Chaotic Modeling and Simulation, 2013.

HENSON, M. A. Nonlinear model predictive control: current status and future directions. Computers and Chemical Engineering, v. 23, 1998. 
HERAS-CERVANTES, M. et al. Analysis and Comparison of a Non-linear Model and a Model Based on Neural Networks to Estimate the Composition in a Distillation Column. IEEE International Autumn Meeting on Power, Electronics and Computing. Ixtapa, Mexico, 2018.

HIEU, D. X. et al. Temperature prediction and model predictive control (MPC) of a distillation column using an artificial neural network based model. International Conference on System Science and Engineering, 2017.

HUANG, H.; RIGGS J. B. On applying for 4x4 distillation control. Advanced Control of Chemical Processes. Pisa, Italy, 2000.

ITO, E. H. C. Desenvolvimento de um sensor virtual de composição em coluna de destilação para aplicação em tempo real. Dissertação (Mestrado em Engenharia Química) - COPPE, Universidade Federal do Rio de Janeiro, Rio de Janeiro, 2014.

JAZAYERI-RAD, $\mathrm{H}$. The nonlinear model-predictive control of a chemical plant using multiple neural networks. Neural Computing \& Application, 2004.

KANTHASAMY, R.; ANWARUDDIN, H.; SINNADURAI, S. K. A New Approach to the Identification of Distillation Column Based on Hammerstein Model. Modelling and Simulation in Engineering, 2014.

KATARIA, G.; SINGH, K. Recurrent Neural Network based Soft Sensor for Monitoring and Controlling a Reactive Distillation Column. Chemical Product and Process Modeling, 2018.

LARSSON, T. SKOGESTAD, S. Plantwide control - A review and a new design procedure. Modeling, Identification and Control, 2000.

ŁAWRYŃCZUK M.; TATAJEWSKI P. An Efficient Nonlinear Predictive Control Algorithm with Neural Models and Its Application to a High-Purity Distillation Process. Artificial Intelligence and Soft Computing. Springer, Berlin, Heidelberg, 2006.

$Ł A W R Y N{ }^{\prime} C Z U K M$ M. Explicit Nonlinear Predictive Control of a Distillation Column Based on Neural Models. Chem. Eng. Technol, 2009.

MACMURRAY, J. C.; HIMMELBLAU, D. M. Modeling and control of a packed distillation column using artificial neural networks. Computers chem. Engng Vol. 19. Elsevier, 1995. 
LUYBEN, W. L. Distillation design and control using aspen simulation. John Wiley \& Sons Inc., 2006.

MARUSAK, P. M. On Prediction Generation in Efficient MPC Algorithms Based on Fuzzy Hammerstein Models. Artificial Intelligence and Soft Computing. Springer, Berlin, Heidelberg, 2010.

MIYOSHI, S. C. Controle preditivo não-linear baseado em modelo orientado a blocos. Tese (Doutorado em Engenharia Química) - COPPE, Universidade Federal do Rio de Janeiro, Rio de Janeiro, 2016.

NORMEY-RICO, J. E. et al. Utilização da Técnica de Controle Preditivo MultiVariável GPC no Controle de Temperatura de Topo e Fundo de uma coluna de destilação depropanizadora. $3^{\circ}$ Congresso Brasileiro de P\&D em Petróleo e Gás, 2005.

PEARSON, R. K.; OGUNNAIKE, B. A. Nonlinear process identification. Nonlinear process control. Englewood Cliffs, NJ: Prentice-Hall, 1997.

QIN, S. J.; BADGWELL, T. A. An overview of industrial model predictive control technology. Industrial Model Predictive Control Technology, 1997.

RAMLI, N. M.; HUSSAIN, M. A.; JAN, B. M. Multivariable control of a debutanizer column using equation based artificial neural network model inverse control strategies. Neurocomputing, Elsevier, 2016.

RANGARATNAM, B.; RIGGS, J. B. A novel Hammerstein model applied for high puruty distillation control. Dynamics and Control of Process Systems, Corfu, Greece, 1998.

RAO, C. V.; RAWLINGS, J. B. Constrained process monitoring: Movinghorizon approach. AIChE journal, v. 48, n. 1, p. 97-109, 2002.

$\mathrm{RECH}$, E. Estudo da estratégia de controle regulatório de uma coluna despentanizadora. Universidade Federal do Rio Grande do Sul, Departamento de Engenharia Química, 2013.

RICHALET, J. et al. Model predictive heuristic control: Applications to industrial processes. Automatica, v. XIV, n. 5, p. 413-428, 1978.

SALLES, A; NUNES, M. Controle Preditivo de um processo de produção de óleo e gás através de gas-lift. Trabalho de Conclusão de Curso. (Graduação em Engenharia Química) - Pontifícia Universidade Católica do Rio de Janeiro, 2017. 
SANTOSO, H.; BAO, J.; LEE, P. (2009). Operability Analysis of MTBE Reactive Distillation Column using a Process Simulator. Chemical Product and Process Modeling, 2019.

SEBORG, D. E.; EDGAR, T. F.; MELLICHAMP, D. A. Process Dynamics and Control. 3. ed. Hoboken NJ: John Wiley \& Sons Inc., 2010.

SEBORG, D. E. et al. Process Dynamics and Control. John Wiley \& Sons Inc., 2011.

SHARMA, N.; SINGH, K. Model predictive control and neural network predictive control of TAME reactive distillation column. Chemical Engineering and Processing: Process Intensification. Elsevier, 2012.

SHARMA, N.; SINGH, K. Neural network and support vector machine predictive control of tert-amyl methyl ether reactive distillation column. Systems Science \& Control Engineering: An Open Access Journal, 2013.

SHOBRYS, D.; WHITE, D. Planning scheduling and control systems: why cannot they work together. Computers and Chemical Engineering, 26, 2002. 149-160.

SILVA, I. N. et al. Artificial Neural Networks: A practical Course. Springer, São Paulo, 2017.

SINGH, A. K., TYAGI, B., KUMAR, V. Classical and Neural Network-Based Approach of Model Predictive Control for Binary Continuous Distillation Column. Chem Product Process Model 2014;9:71-87. doi: 10.1515/cppm-20130013.

SKOGESTAD, S. The dos and dont's of distillation column control. Institution of Chemical Engineer, 2007.

VAZ, C. E. M.; MAIA, J.L.P.; SANTOS, W. G. Tecnologia da Indústria do Gás Natural. $1^{\text {a }}$ ed., Blucher, 2009.

WAHID, A. Improved multi model predictive control for distillation column. Thesis in Chemical Engineering, Faculty of Chemical and Energy Engineering, Universiti Teknologi Malaysia, 2016.

WANG, L. Model predictive control system design and implementation using MATLAB. Springer, 2009. 
YUSOFF, Z. M. et al. Modeling of steam distillation system using Hammerstein-Wiener model. IEEE 7th International Colloquium on Signal Processing and its Applications, 2011. 